\title{
The Zygoma Anatomy-Guided Approach for Placement of Zygomatic Implants
}

\author{
Carlos Aparicio, MD, DDS, MSc, MSc, DLT, PhD ${ }^{a, *}$, Waldemar D. Polido, DDS, MS, PhD ${ }^{b}$, \\ Hooman M. Zarrinkelk, DDS ${ }^{\mathrm{C}}$
}

\section{KEYWORDS \\ - Zygoma anatomy-guided approach $\bullet$ ZAGA $\bullet$ ZAGA concept $\bullet$ tunnel osteotomy $\bullet$ Channel osteotomy $\bullet$ Extramaxillary implants}

\section{KEY POINTS}

- The essence of the ZAGA Concept is to provide the patient with a zygomatic anchored rehabilitation according to their specific anatomy.

- ZAGA Concept includes the choice of the adequate implant design able to adapt to the performed osteotomy.

- The ZAGA concept recommends aiming for a tunnel osteotomy, whenever possible, regardless of the maxillary wall curvature.

- A channel osteotomy is a groove made on the coronal-alveolar bone and sometimes also in the lateral maxillary wall and zygomatic buttress.

- It is critical to preserve the sinus membrane integrity and as much bone thickness as possible at the zygomatic implant critical zone crest level.

\section{The zygomatic anatomy-guided approach concept}

The zygomatic anatomy-guided approach (ZAGA) was described as a refinement of the extrasinus technique for placement of zygomatic implants (ZI). ${ }^{1}$ The concept seeks a patient-specific therapy and applies to all the atrophic maxillary anatomies. According to the ZAGA concept, placement of the $\mathrm{ZI}$ is guided by the anatomic and prosthetic requirements. The osteotomy goals (Box 1) are to achieve maximum primary stability, optimal anteroposterior (AP) distribution, and implant trajectory and position that prevent potential longterm complications, such as oral-antral fistula formation or soft tissue dehiscence (Figs. 1-113).

The essence of the ZAGA concept is understanding the possible anatomic variations present between patients, and even in different sites for the same patient, and then to provide this patient with a zygomatic anchored rehabilitation according to the specific anatomy. ZAGA concept includes adaptation of the implant trajectory to the anatomy and the choice of the adequate implant design able to adapt to the performed osteotomy.

In execution of the ZAGA concept, the implant path may be intrasinus, extrasinus (see Figs. 21-55), or in multiple

\footnotetext{
${ }^{a}$ Zygomatic Unit at Hepler Bone Clinic, ZAGA Center Barcelona, Roman Macaya, 22-24, Barcelona 08022, Spain

b ZAGA Center, Oral and Maxillofacial Surgery, Indiana University School of Dentistry, Indiana University, 1121 West Michigan Street, Indianapolis, IN 46202, USA

c ZAGA Center Los Angeles, American Board of Oral and Maxillofacial Surgery, Private Practice, 5200 telegraph rd, Suite B, Ventura, CA 93003, USA

* Corresponding author.

E-mail address: carlos.aparicio@zagacenters.com
}

intermediary positions (see Figs. 1-20 and 56-113), using the maxillary wall as an additional source of anchorage. The ZAGA concept aims to maximize the primary stability of a prosthetically ideal positioned $\mathrm{ZI}$, which produces a conservative osteotomy at the coronal-alveolar, medial-maxillary wall, and apical-zygomatic bone level.

Once the anatomic features of that specific patient have been visualized and studied, the ZAGA concept provides the clinician with decision-making tools on the establishment of the ideal coronal entrance point at the alveolar process and the apical entry into the zygomatic bone.

\section{Late complications of the zygoma-related rehabilitation}

The authors propose the term, late complications, for those complications appearing at least 3 months after surgery, once implants have been individually tested for no rotational movements, pain, or infection.

The zygoma fixture-associated late complications frequently are related to the surgical technique; that is, extramaxillary placement of ZI may lead to an increased possibility for mucosal dehiscence compared with the classical palatal perforation. ${ }^{1}$ On the other hand, intrasinus techniques have more possibilities of developing an oral-antral fistula. ${ }^{2,3}$ An intrasinus implant path perforates the sinus through a thinner bone and thicker mucosa, unable to be sealed by the abutment (see Figs. 22-24). The intrasinus path together with an increased anterior maxillary wall concavity also is associated with implant platforms positioned at averages of $12 \mathrm{~mm}$ palatal to the crest. The resulting bulky prostheses provokes hygiene difficulties and soft tissue problems like gingival hyperplasia or/and infection, which may lead to bone resorption around the implant neck at the 


\section{Box 1. The ZAGA minimally invasive zygo- matic osteotomy goals}

- Accomplish a prosthetically driven implant trajectory, placing the implant head at the optimal dental position.

- Achieve optimal AP distribution of the implants.

- Achieve maximal implant primary stability.

- Preserve as much bone as possible at the maxillary wall and alveolar bone.

- Maximize the BIC along the length of the whole implant. This includes alveolar, maxillary wall, and zygomatic bone.

- Complete sealing of the osteotomy by the implant body.

- Protect the sinus integrity at the implant head/neck level to prevent late sinus-oral communication.

- Prevent soft tissue dehiscence.

palatal region. Resorption of the thin palatal bone rapidly leads to oral-antral fistula.

\section{The zygomatic anatomy-guided approach concept for minimally invasive osteotomy}

\section{The alveolar tunnel osteotomy for a round section implant design}

Following the ZAGA concept for a patient-specific zygomatic therapy, if the cone-beam computer tomography (CBCT) crosssectional plane for virtual implant placement shows that residual bone at the sinus floor level is sufficient in thickness and width (minimum, 4-mm high $\times 6-\mathrm{mm}$ wide), the Zl should enter the antrum through the residual alveolus. In other words,

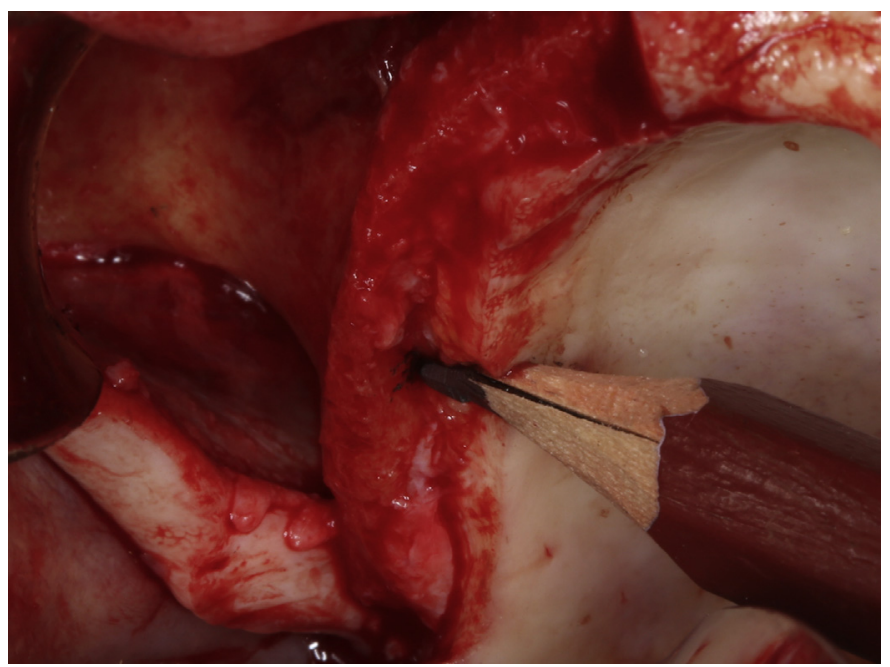

Fig. 2 Case 1: both coronal and zygomatic entrances are marked previous to drilling.

whenever bone architecture at the desired implant platform position is sufficient to circumferentially enclose the implant neck with at least $4 \mathrm{~mm}$ of bone, attempts should be made to maintain this bone and to place the implant through it (ZAGA types 0 and 1 ). This concept minimizes the risk of late soft tissue complications.

The authors naming the circular osteotomy a tunnel osteotomy because, at the alveolar osseous entrance, it has a floor, lateral walls, and a more or less complete roof. The ZAGA concept recommends aiming for a tunnel osteotomy whenever possible, regardless of the maxillary wall curvature (see Figs. 1-20) (ZAGA types 0,1 , and 3). In other words, in the presence of a remaining alveolar crest allowing for a tunnel-type perforation, a maxillary wall curvature with a pronounced

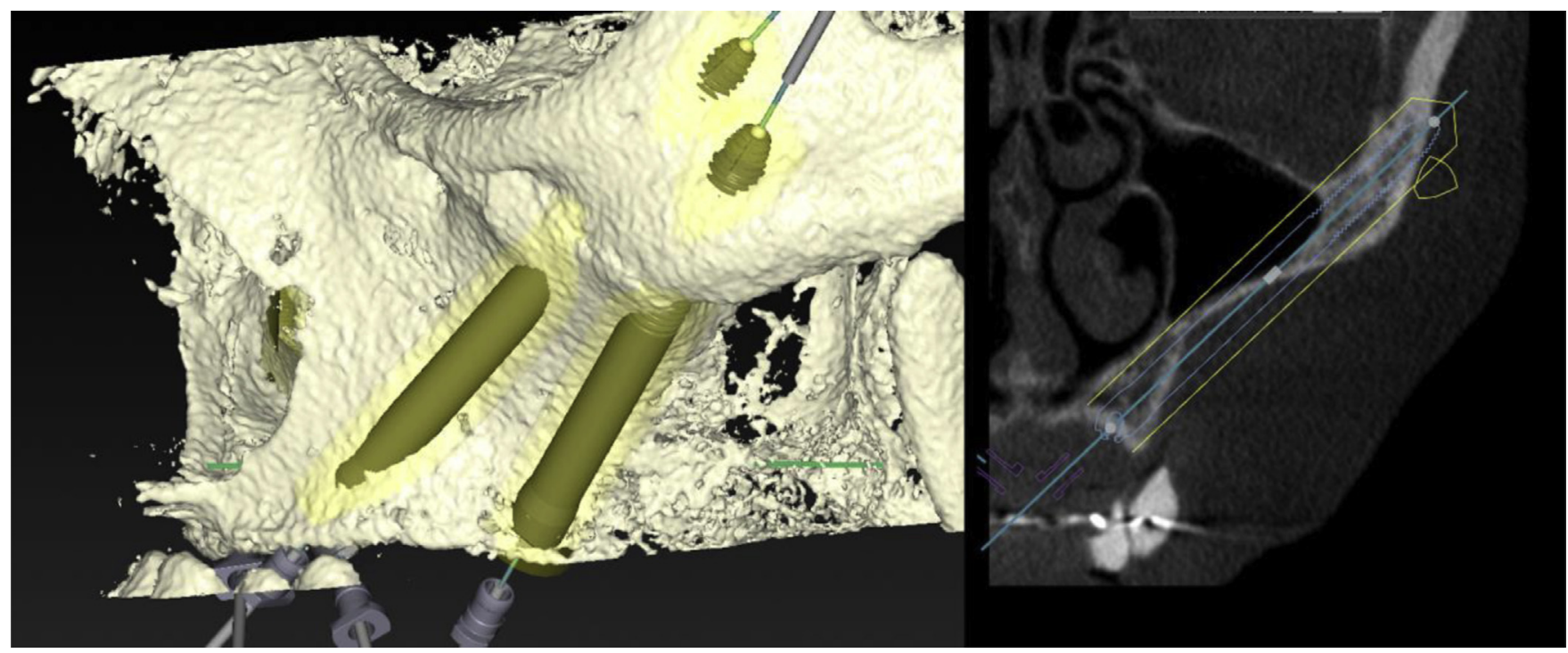

Fig. 1 Simulation of an extrasinus path in a ZAGA type 3 situation. Implant path will use a ZAGA tunnel-type osteotomy to reach the zygoma. 


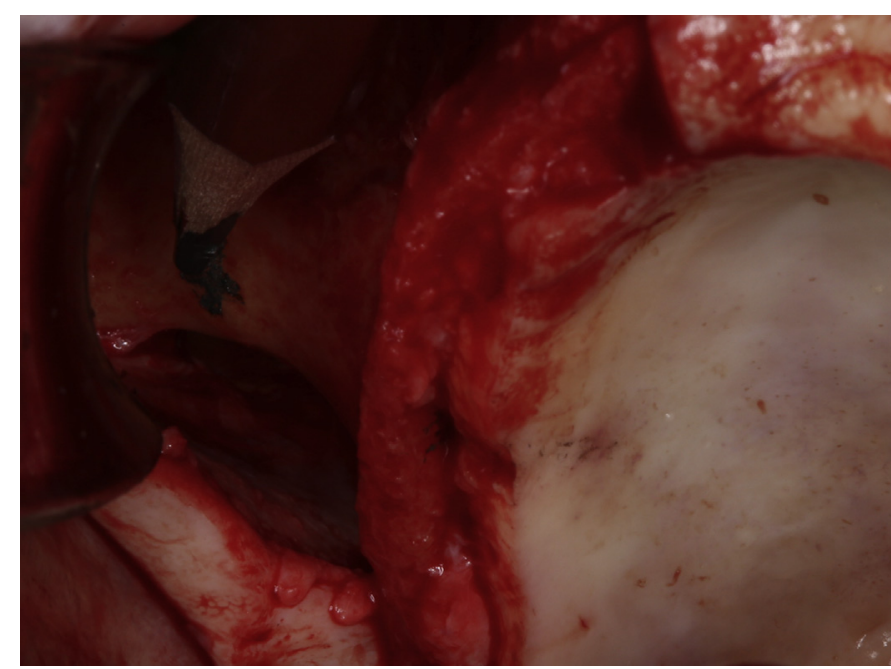

Fig. 3 Case 1: the antrostomy location also is marked.

curvature is not perforated until the antrostomy zone (ZAGA type 3 ). The rationale is that a stable ZI, with an appropriate threaded profile, surrounded by sufficient bone at the alveolar entrance and stabilized by adequate prostheses, will achieve osseointegration capable of long-term sealing the sinus entrance.

Tunnel osteotomy typically is used in ZAGA types 0,1 , and 3 maxillary wall situations when it is accompanied by an adequate thickness and geometry of alveolar bony support circumferential to the implant neck.

Tunnel osteotomy, by definition, has a circular profile entrance that needs to be sealed by a round profile implant.

In order to avoid possible bone loss from implant micromovements, ideally, ZI should be splinted immediately following placement to other conventional or $\mathrm{Zl}$ in a rigid crossarch stabilization system.

Providing that the clinician feels comfortable controlling the drilling direction and trajectory, a lateral window-shaped osteotomy into the maxillary antrum is not recommended to minimize bone injury. The entry point preferably should be

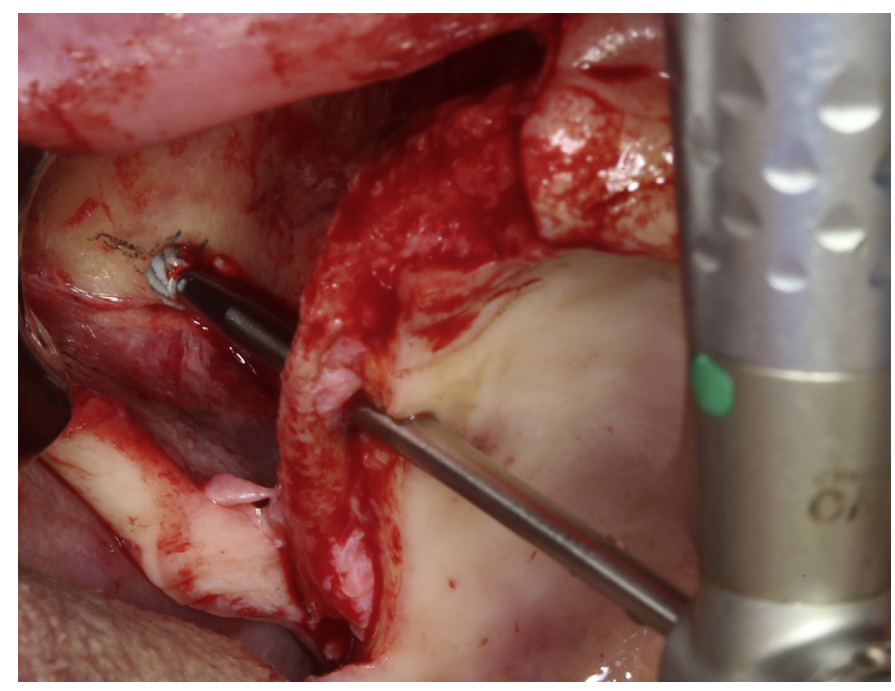

Fig. 5 Case 1: the round bur has crossed alveolar bone. Membrane integrity was maintained.

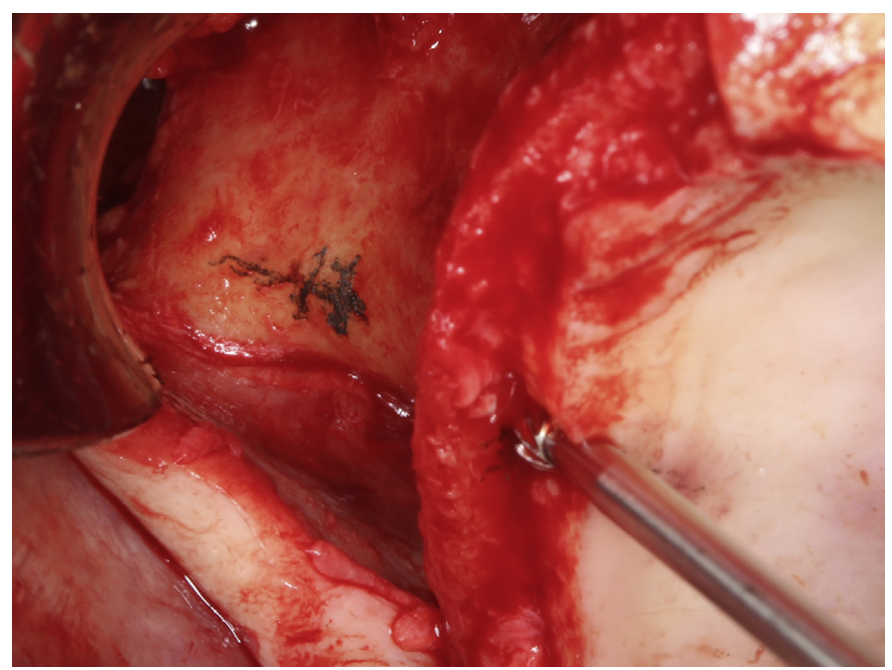

Fig. 4 Case 1: the round bur is used to drill a tunnel on alveolar bone.

located in a position close to the middle part of the alveolar crest regardless of the maxillary wall anatomy, attempting to place the implant neck fully surrounded by the adequate thickness of native bone. The circular osteotomy for implant placement penetrates at least $4 \mathrm{~mm}$ of crest, with no special effort to maintain sinus membrane integrity, and adopts either a total, partial, or extrasinus path on its midpart until reaching the zygomatic bone. The final determination of the relationship between implant and maxillary anterior wall is determined by the curvature of the external wall of the maxillary buttress.

There is no scientific documentation of the minimum amount of residual bone required to withstand the masticatory loads applied from the $\mathrm{ZI}$ to the sinus floor bone-implant junction. Circumstances affecting bone to implant contact (BIC) quality and maintenance at the alveolar entrance can differ dramatically from 1 patient to the next, that is, difference between final drill diameter and implant diameter, implant insertion precision, degree of $\mathrm{ZI}$ stability resisting

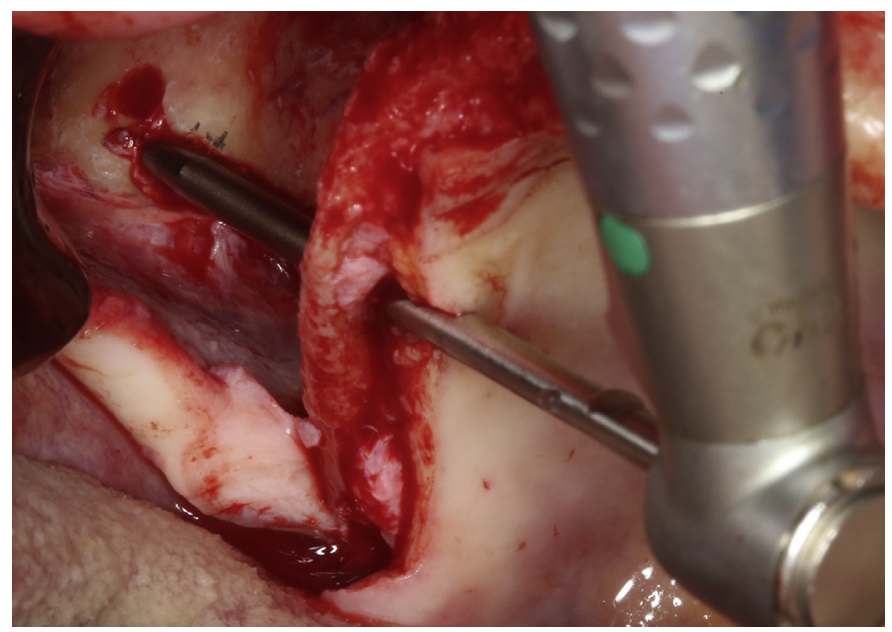

Fig. 6 Case 1: the round bur has perforated the zygomatic process of the maxilla. 


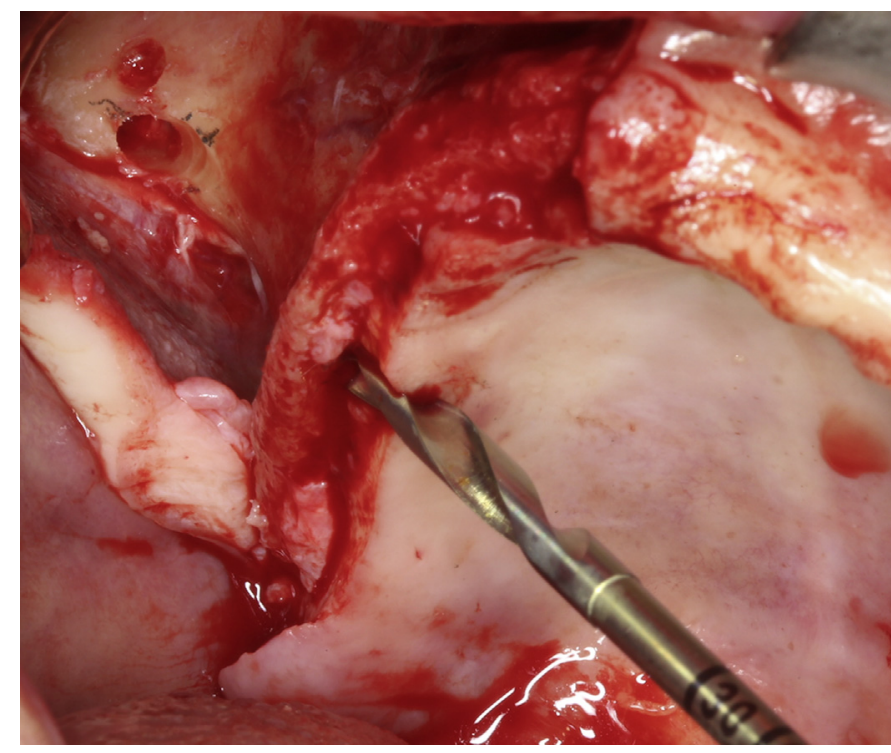

Fig. 7 Case 1: a stepped Straumann AG 2.9-mm diameter twist drill was used as a final drill.

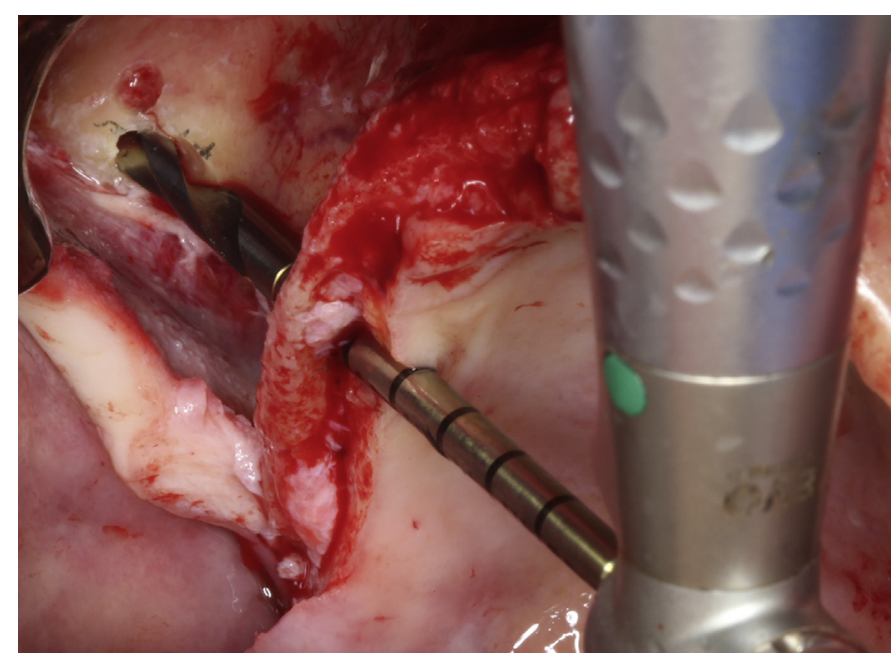

Fig. 9 Case 1: the twist drill attacks the zygomatic bone using a sliding movement supported by the alveolar tunnel and the channel.

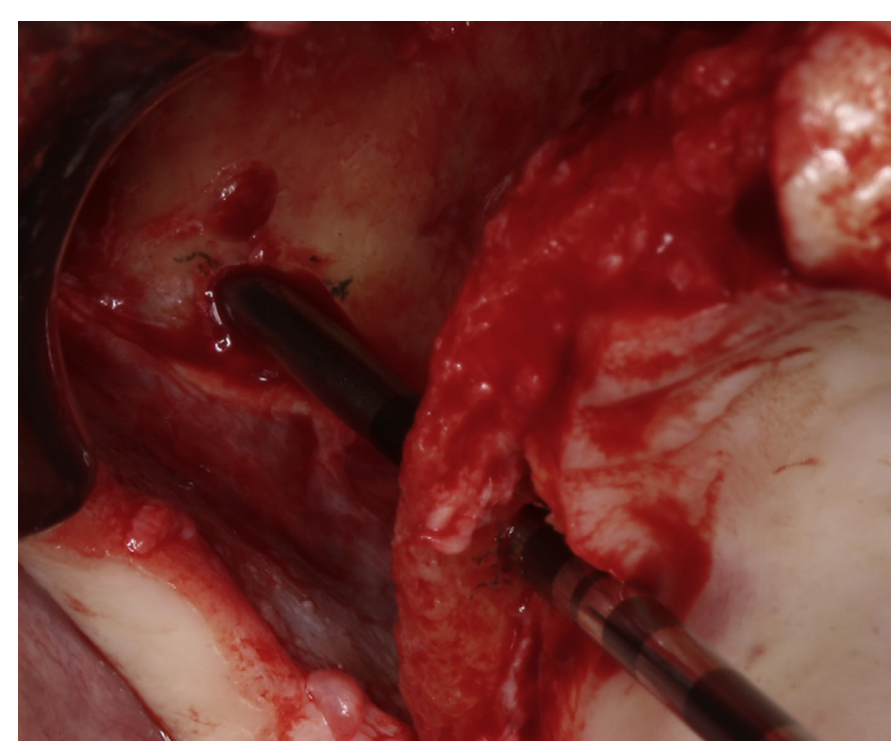

Fig. 11 Case 1: a Straumann AG zygomatic double gauge is used to measure the osteotomy length.

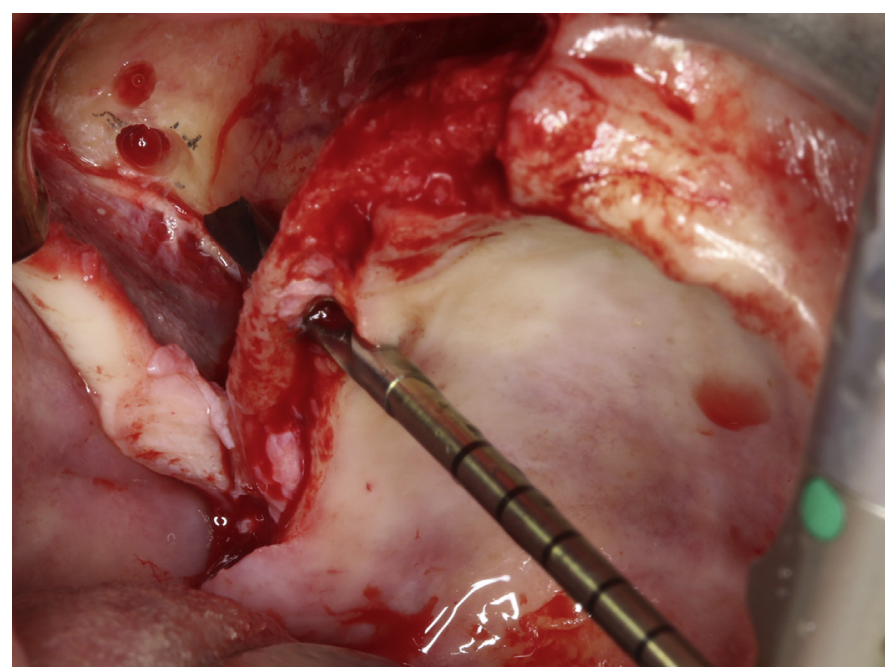

Fig. 8 Case 1: maxillary wall should be prepared evenly. When there is no roof, drills may trend to drill the air.

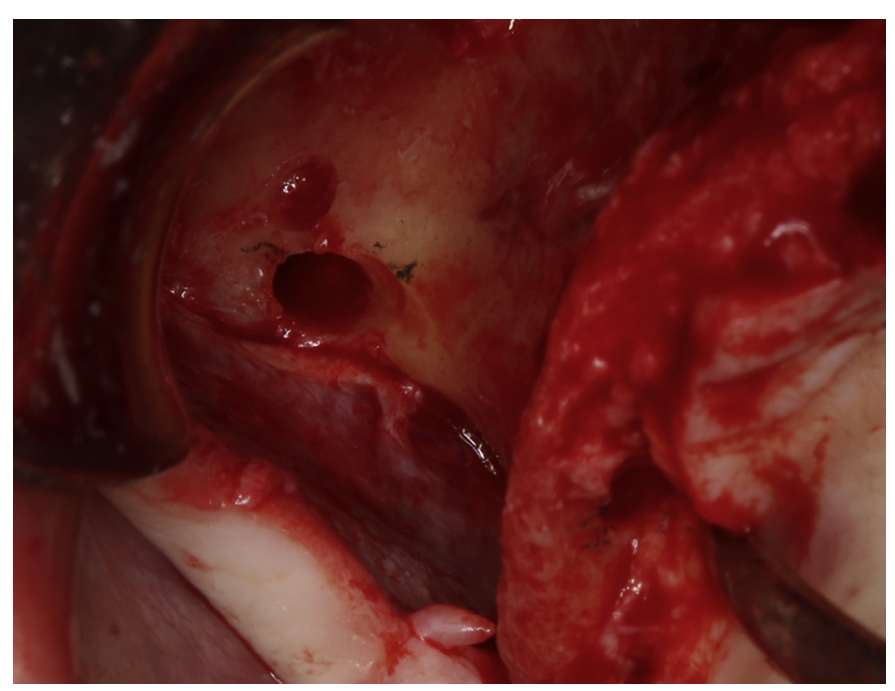

Fig. 10 Case 1: the ZAGA tunnel type of osteotomy respects sinus integrity at the ZICZ.

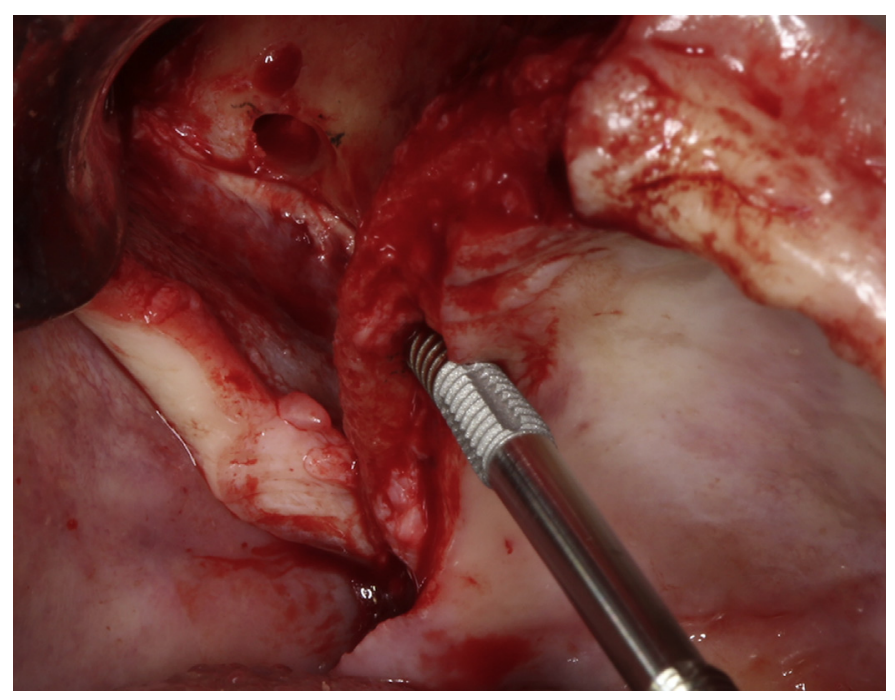

Fig. 12 Case 1: a circular section implant (Straumann ZAGA Round) is self-tapping the alveolar tunnel osteotomy. 


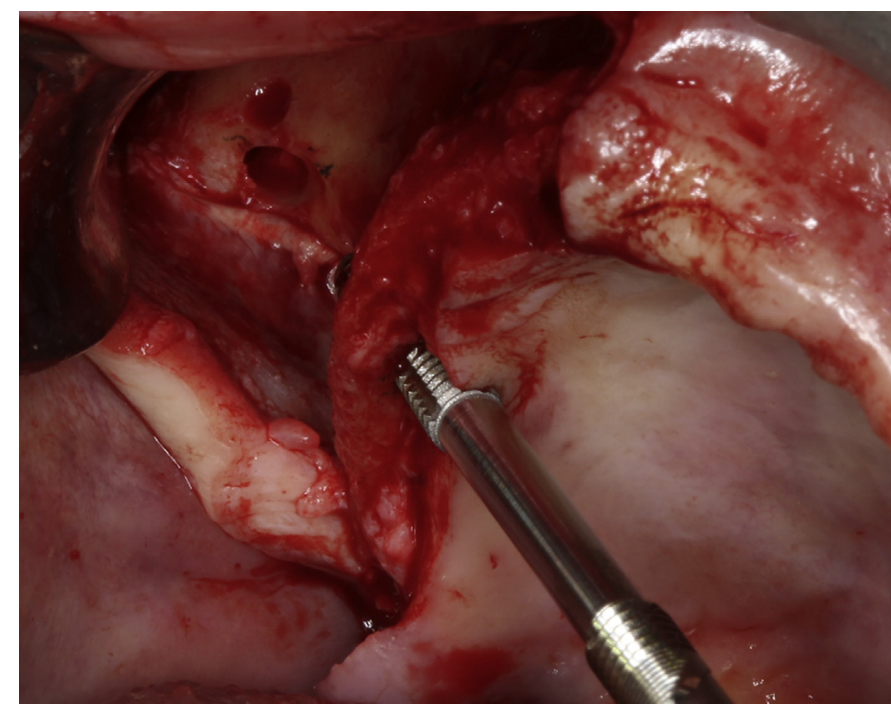

Fig. 13 Case 1: due to the implant (Straumann ZAGA Round) self-tapping design, BIC is maximized and no gaps are left between alveolar bone and the implant.

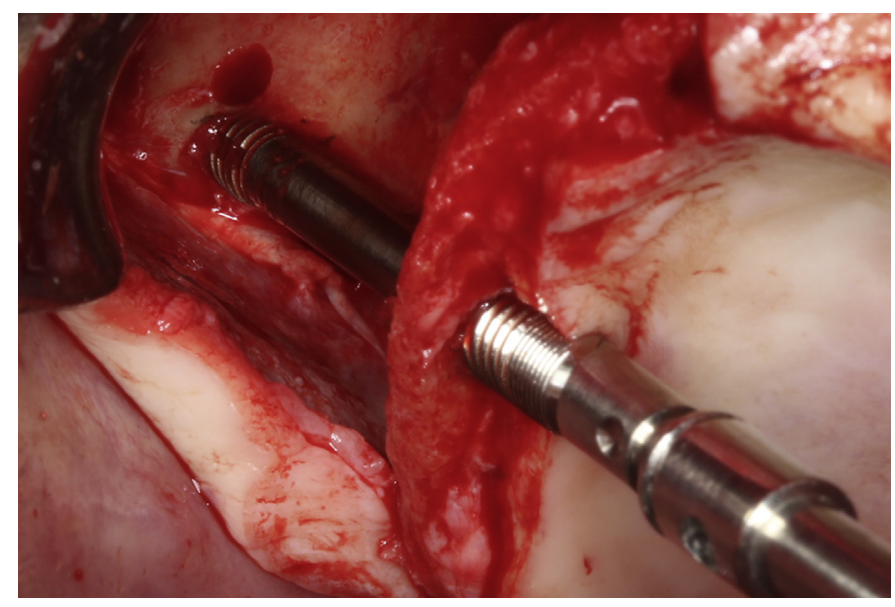

Fig. 15 Case 1: the implant (Straumann ZAGA Round) neck has macrothreads to help bone integration. The bony side of the implant head has microthreads for bony stabilization.

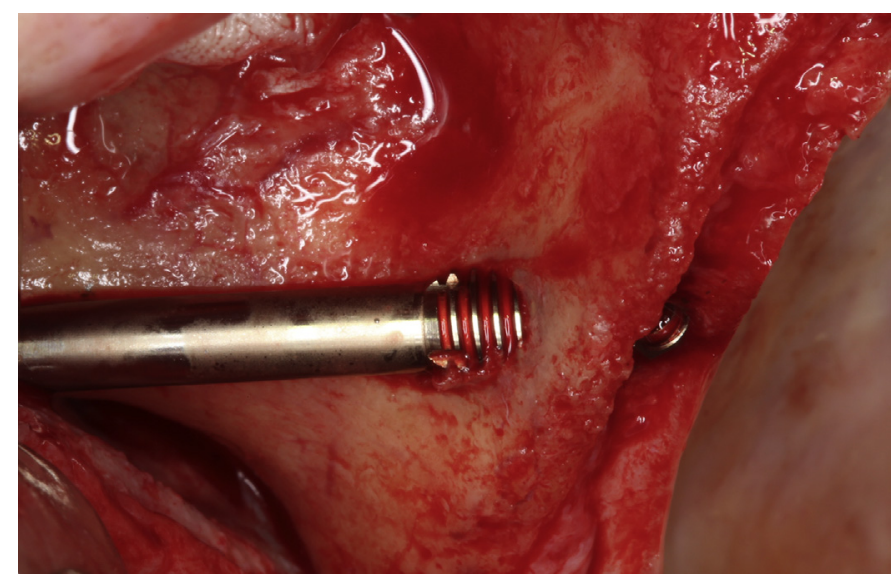

Fig. 17 Case 1: lateral view of the implant design (Straumann ZAGA Round).

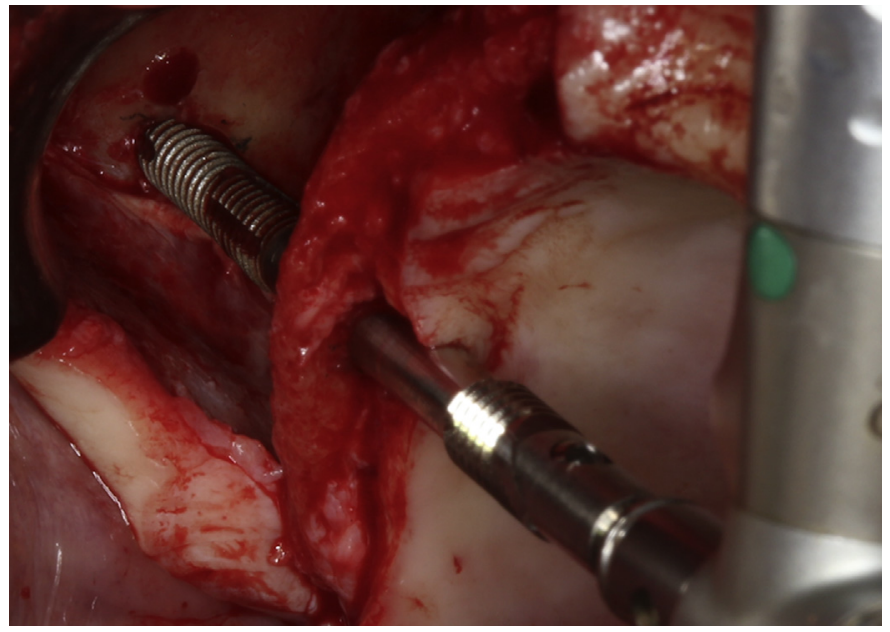

Fig. 14 Case 1: the implant (Straumann ZAGA Round) apical flutes are cutting the zygomatic bone facilitating insertion torque values above $50 \mathrm{Ncm}$.

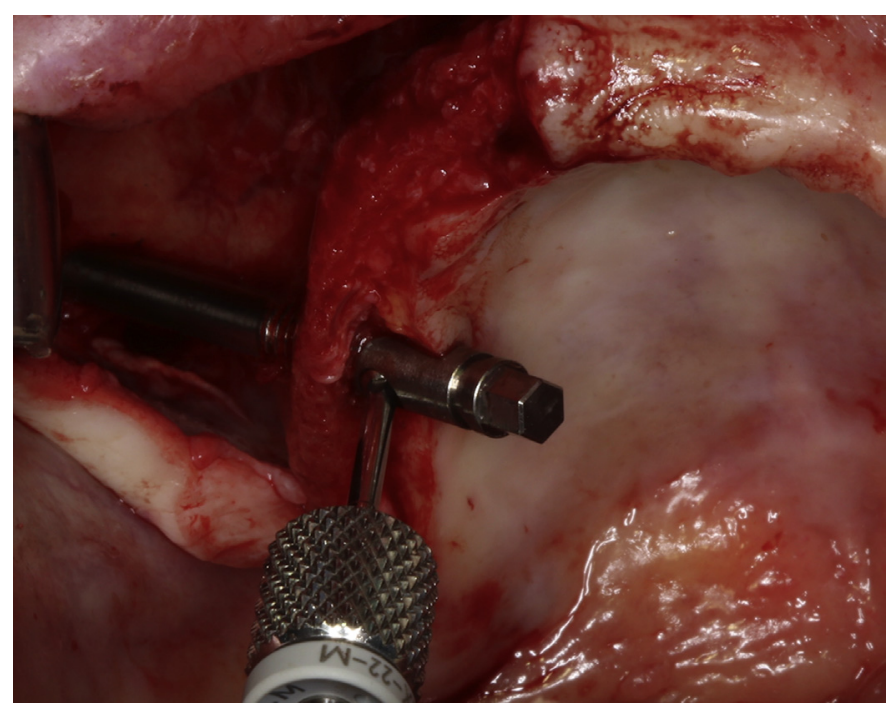

Fig. 16 Case 1: the implant (Straumann ZAGA Round) mount screw gives the direction of the prostheses screw emergence.

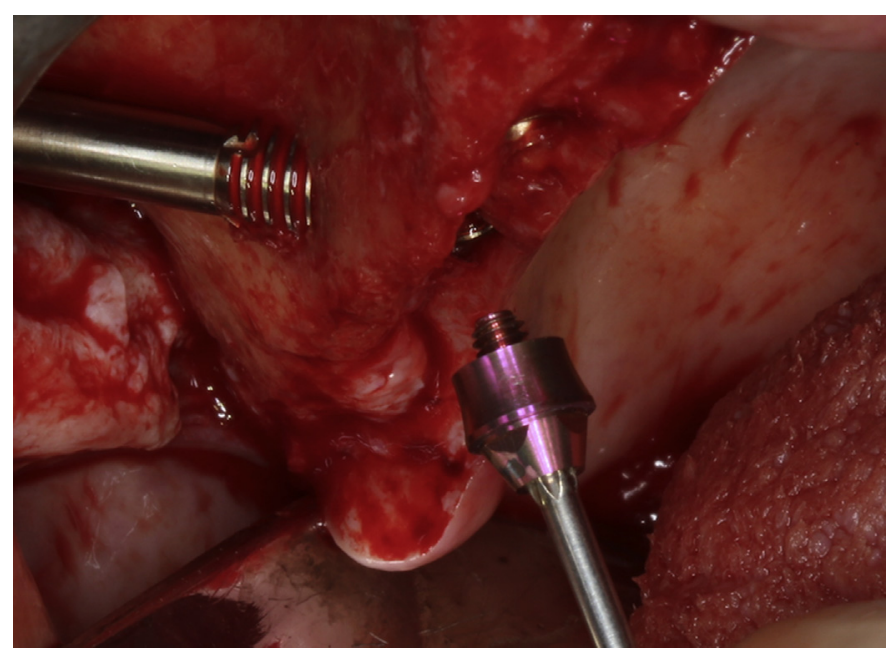

Fig. 18 Case 1: a Straumann abutment is being screwed until reaching $35 \mathrm{~N} \cdot \mathrm{cm}$. 


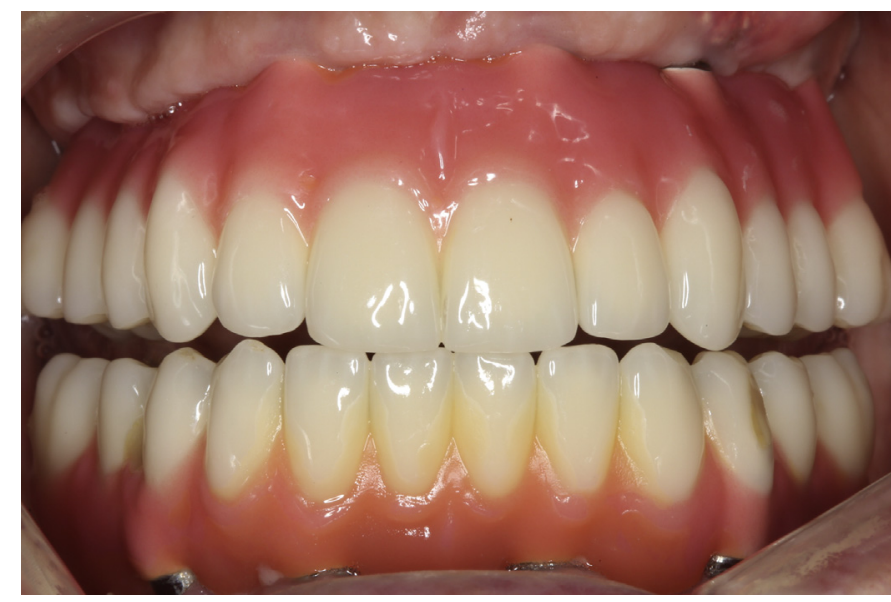

Fig. 19 Case 1: final prostheses and soft tissues 1 year postsurgery. Prostheses placed by Drs Peter and Madalina Simon, ZAGA Center Stuttgart, Germany.

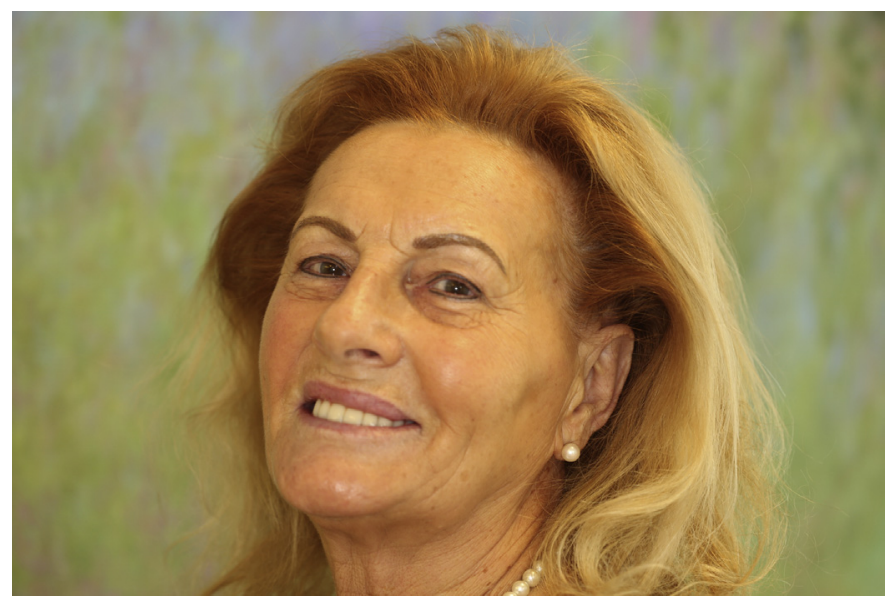

Fig. 20 Case 1: patient satisfaction 1 year postsurgery. Prostheses placed by Drs Peter and Madalina Simon, ZAGA Center Stuttgart, Germany.

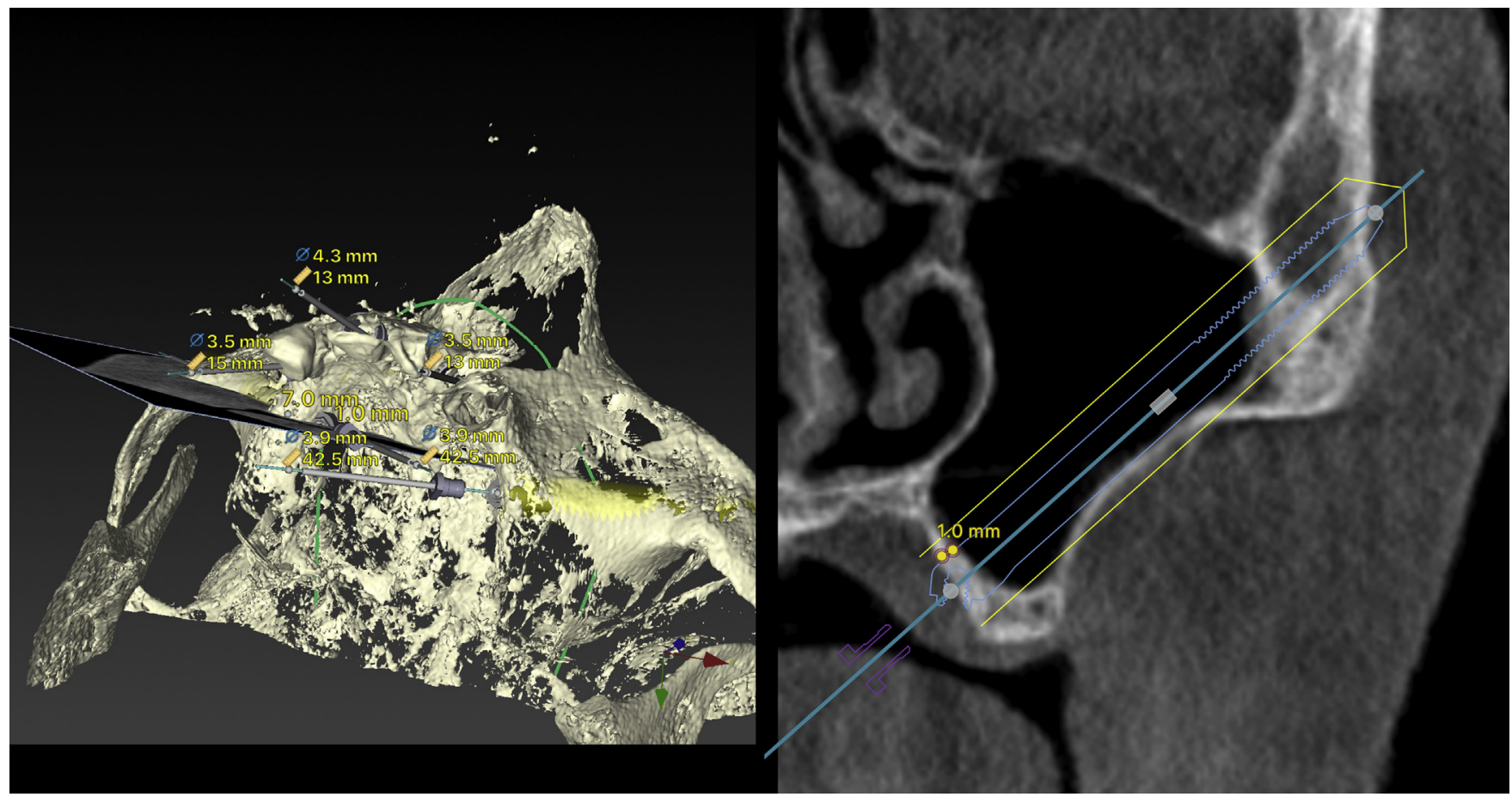

Fig. 21 Case 2: right side 3D and 2D images representing the anatomic features of an eventual ZI trajectory. The planning software simulating an intrasinus classic path. An eventual circular tunnel osteotomy would reach the sinus through scarcely $1 \mathrm{~mm}$ to $2 \mathrm{~mm}$ of alveolar bone thickness. The ZAGA concept recommends visualizing the possibility for bone loss around the implant neck and subsequent development of an oral-antral fistula. 


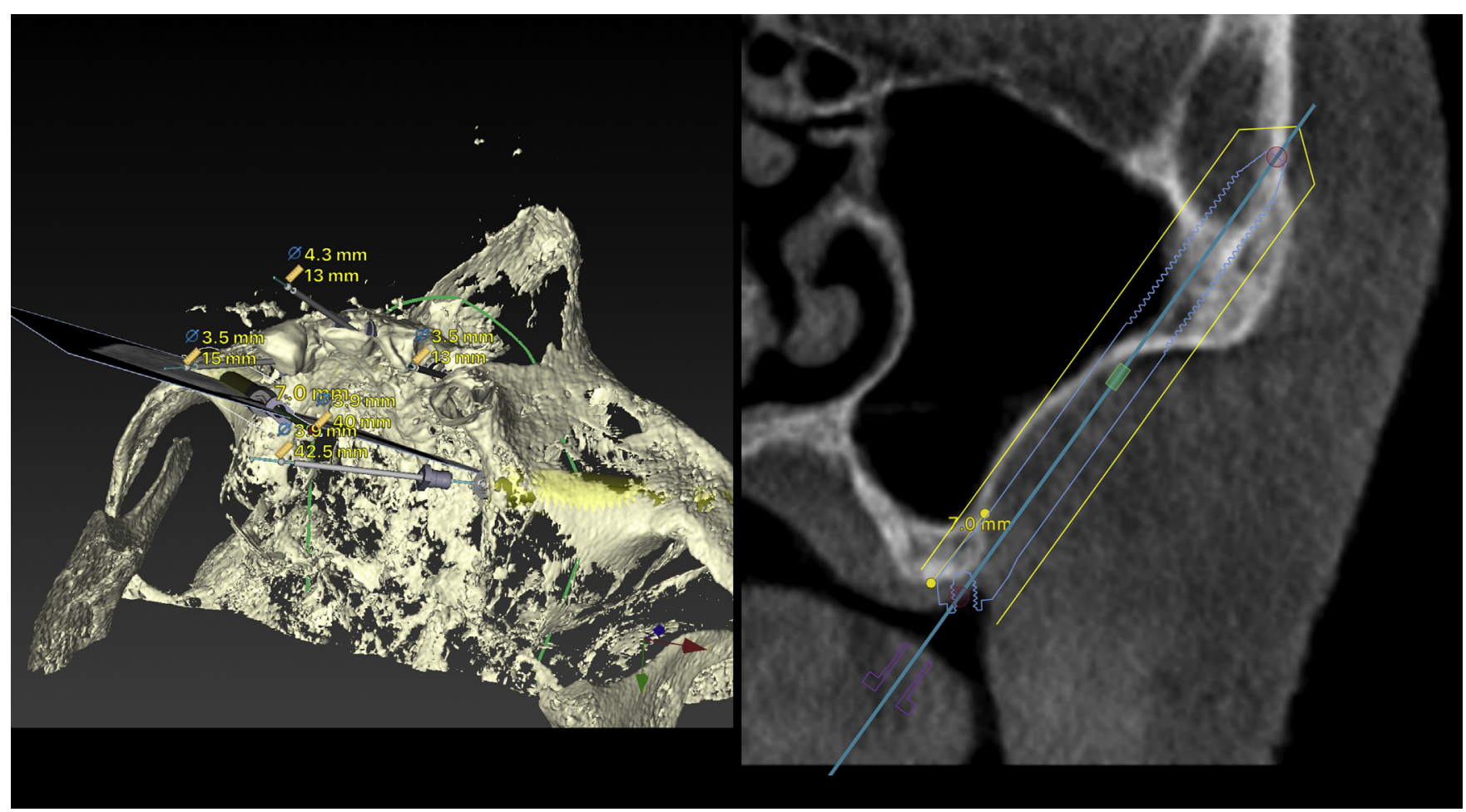

Fig. 22 Case 2: the planning software is simulating an extrasinus path of the same implant of Fig. 21. (right side) According to the ZAGA concept, when facing situations showing less than 4 to $5 \mathrm{~mm}$ thickness at the sinus floor, a more reliable bone sealing and maintenance is achieved by buccal shifting of the osteotomy.

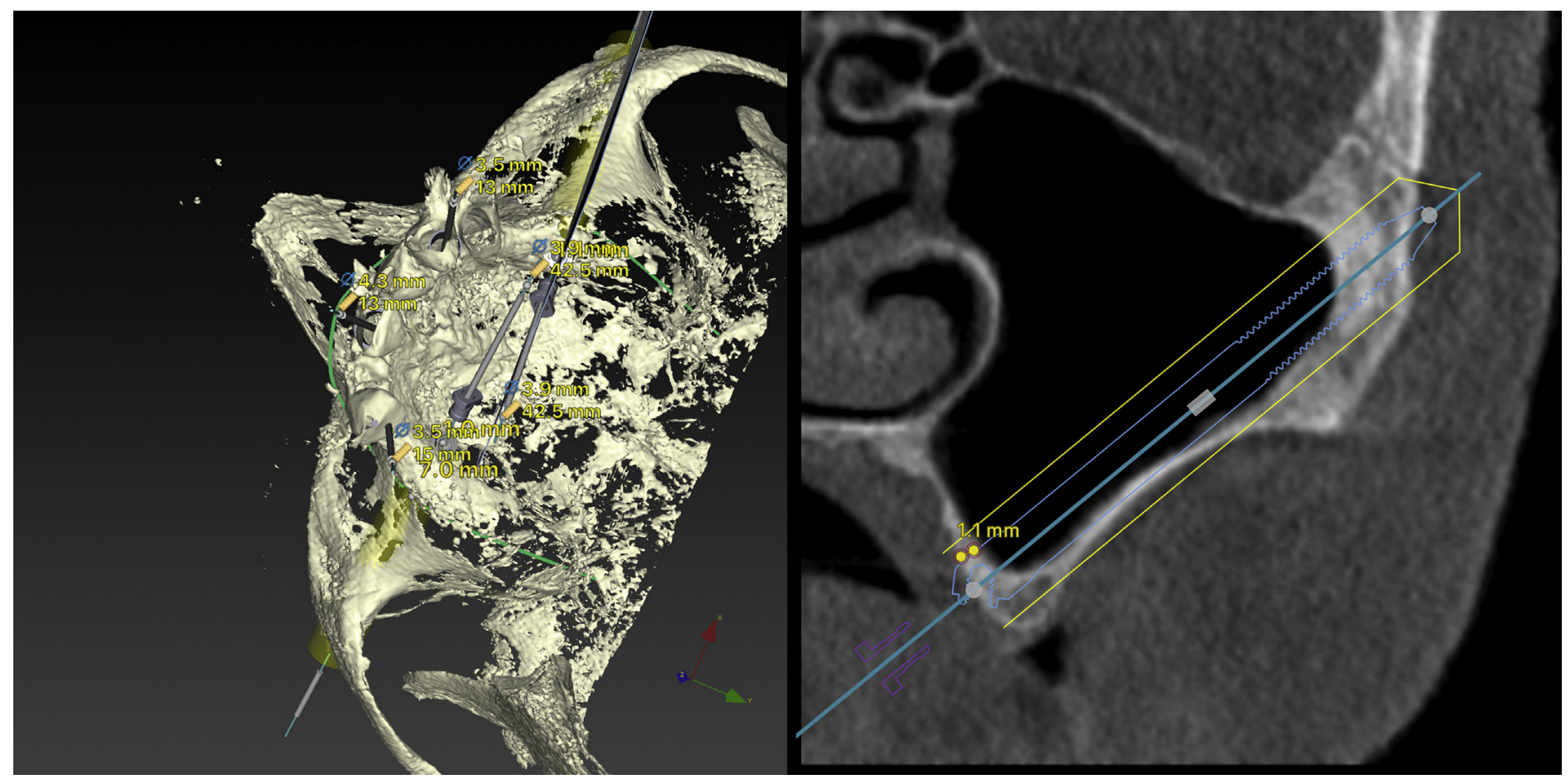

Fig. 23 Case 2: left side 3D and 2D images representing the anatomic features of an eventual ZI trajectory. The planning software simulating the figure is showing an intrasinus planning of the same patient as in Fig. 24. Possibility for late peri-implant bone resorption and oral-antral fistula must be foreseen. 


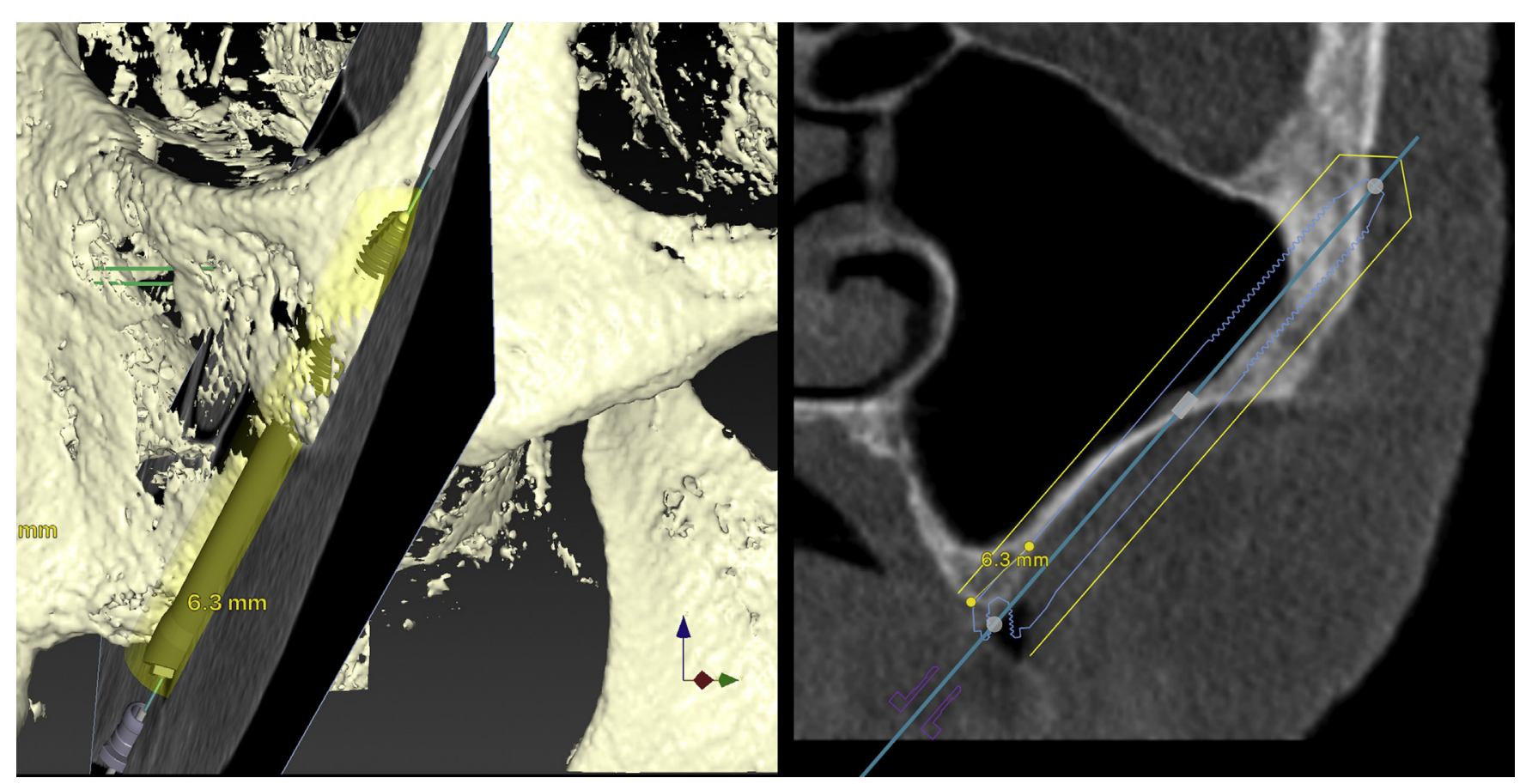

Fig. 24 Case 2: an increased BIC at the ZICZ Is achieved by laterally displacing the osteotomy.

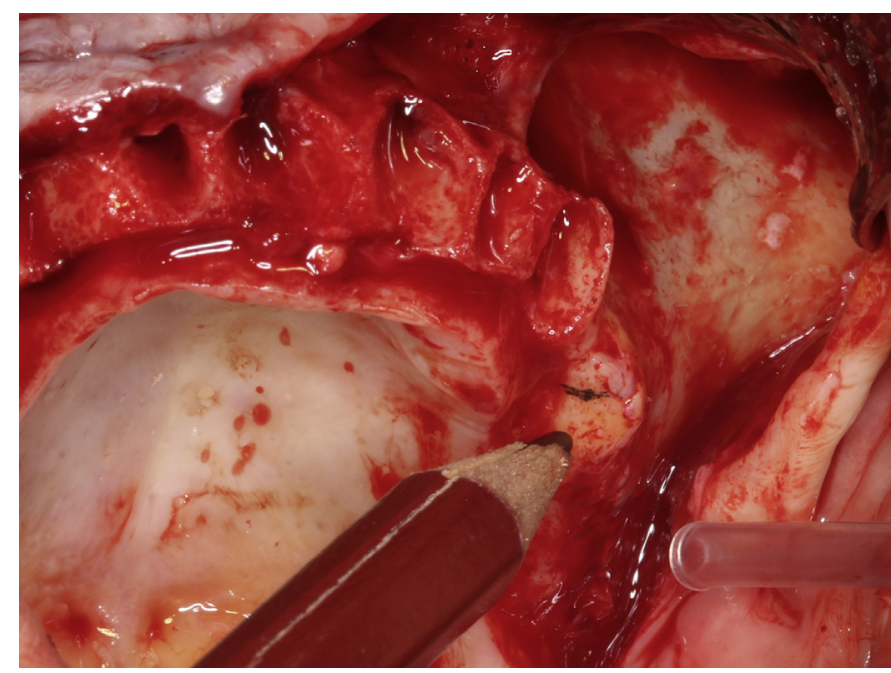

Fig. 25 Case 2: reference points for alveolar and zygomatic bone entrances should be marked.

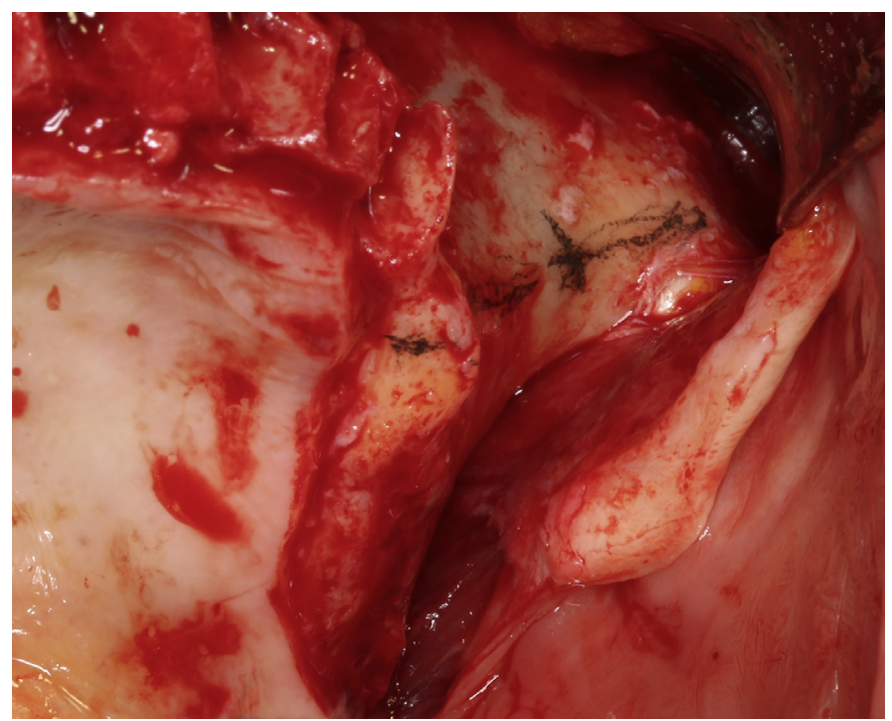

Fig. 26 Case 2: points to be marked are the ZICZ and the antrostomy zone. Eventually, a line joining the ZICZ and the antrostomy points will help by showing the implant path. 


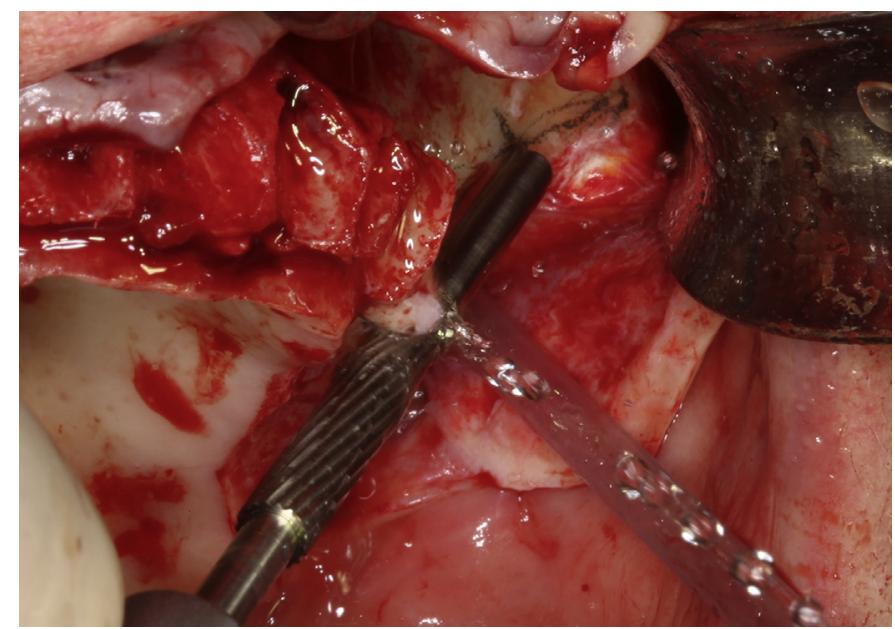

Fig. 27 Case 2: alveolar thickness is less than $4 \mathrm{~mm}$. A ZAGA channel osteotomy was chosen. The lateral cutting bur (Straumann AG) is initiating a narrow channel where the next drill tail will slide.

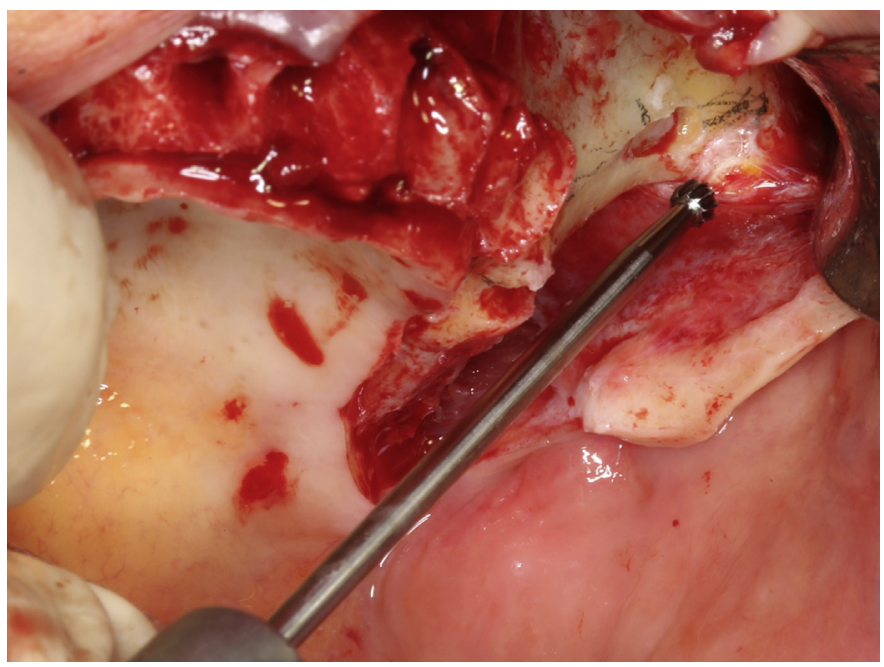

Fig. 29 Case 2: round bur has completed the channel grinding. A notch was marked at the zygomatic process.

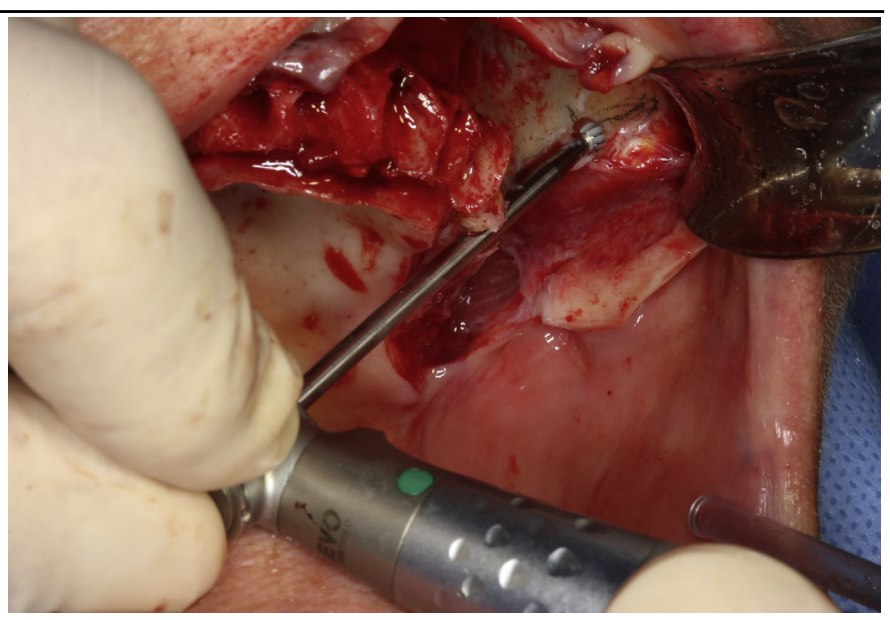

Fig. 28 Case 2: the round bur tail is supported by the alveolar channel. Direction, depth, and inclination of the implant trajectory now should be evaluated. Once the channel shows the desired depth and its floor surface becomes even, the bur tail gently slides up and down using a grinding-back movement, the goal being to prepare a smooth surface supporting the trajectory for the next drill.

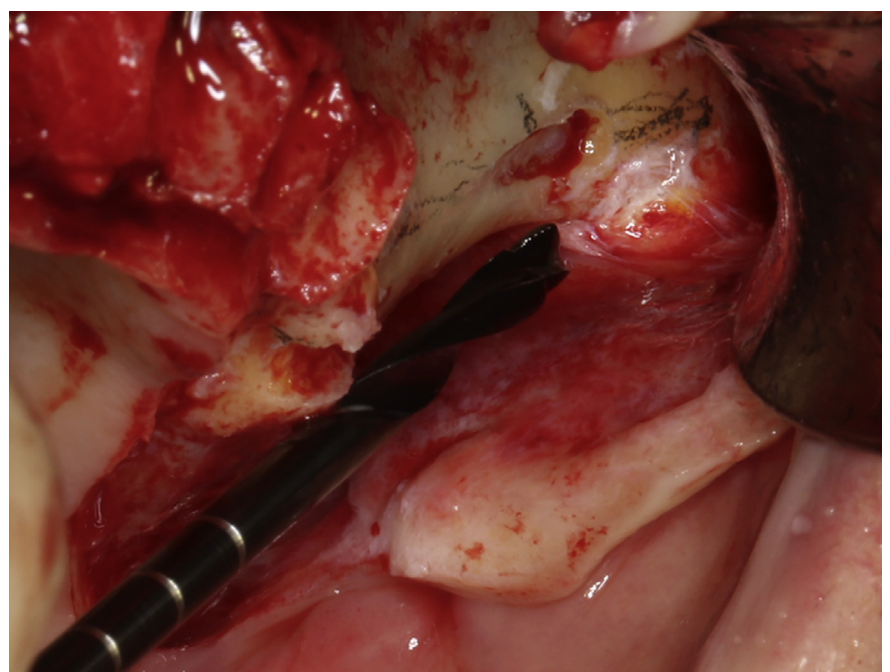

Fig. 30 Case 2: a 3.4-mm diameter implant is planned; conditions of zygomatic bone quality and quantity are not ideal. Then, a twist drill of $2.8-\mathrm{mm}$ diameter is advised as final diameter. 


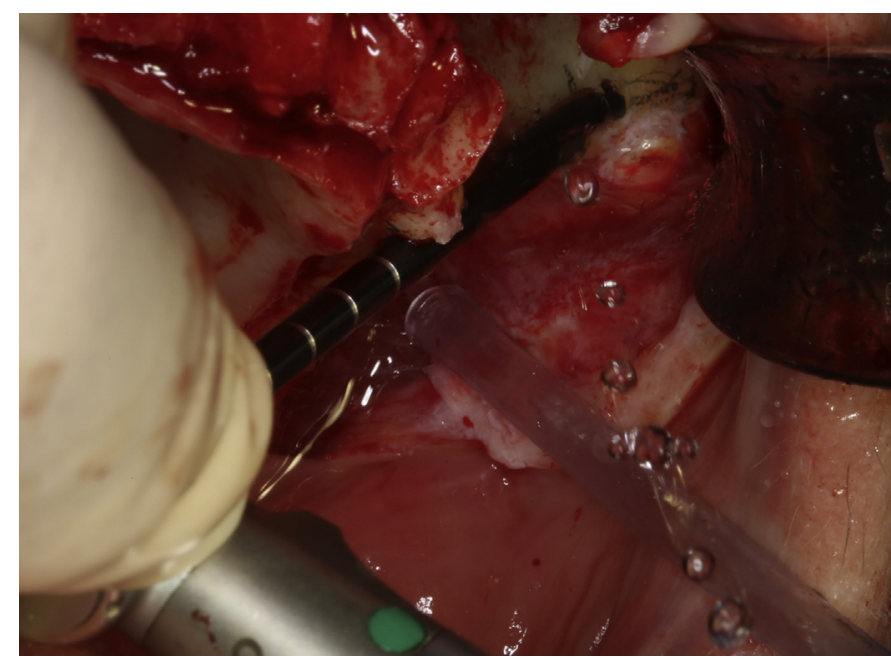

Fig. 31 Case 2: using profuse irrigation, the twist drill slides up and down, using short pressure periods of about 4 seconds, allowing the saline to clean and cool the drill.

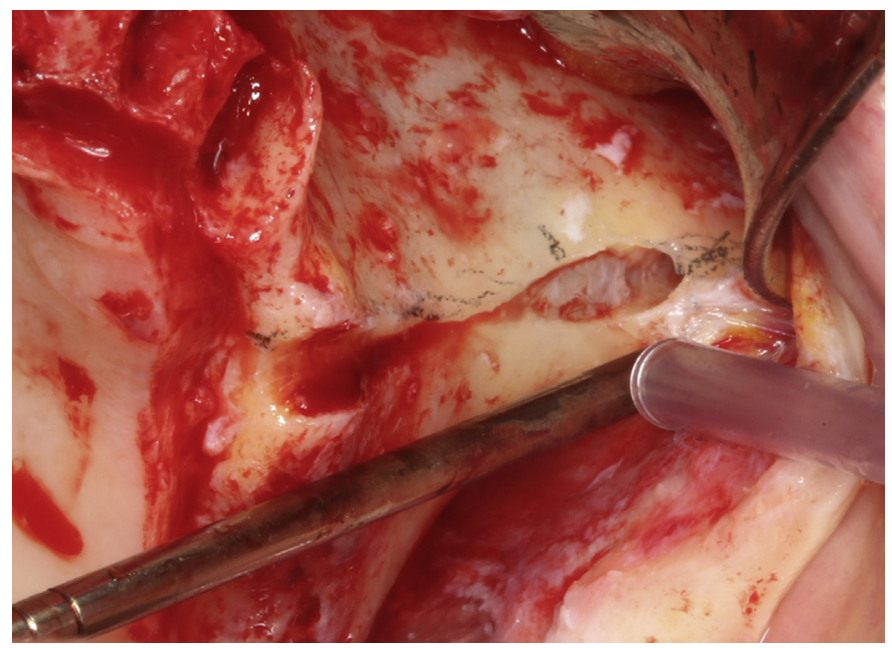

Fig. 32 Case 2: details of the ZAGA channel osteotomy. Minimal destruction of the alveolar bone and maxillary wall has been achieved. The under-preparation totally matches the chosen implant design. Sinus lining integrity has been maintained. The antrostomy zone was moved to an apical position.

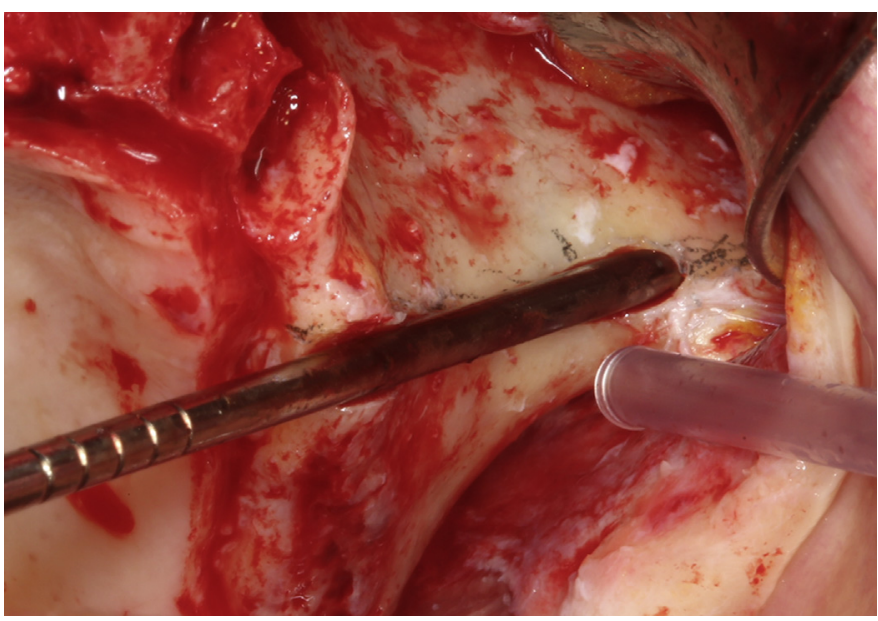

Fig. 33 Case 2: implant length is estimated with a gauge.

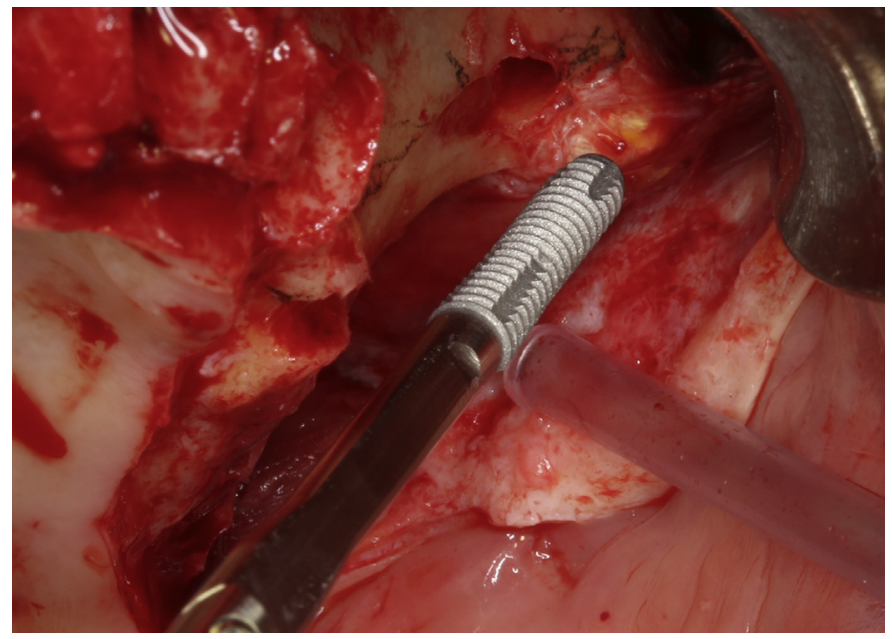

Fig. 34 Case 2: a flat body section implant design (Straumann ZAGA Flat) is presented. The round tapered apical part of the implant is provided with cutting flutes. 


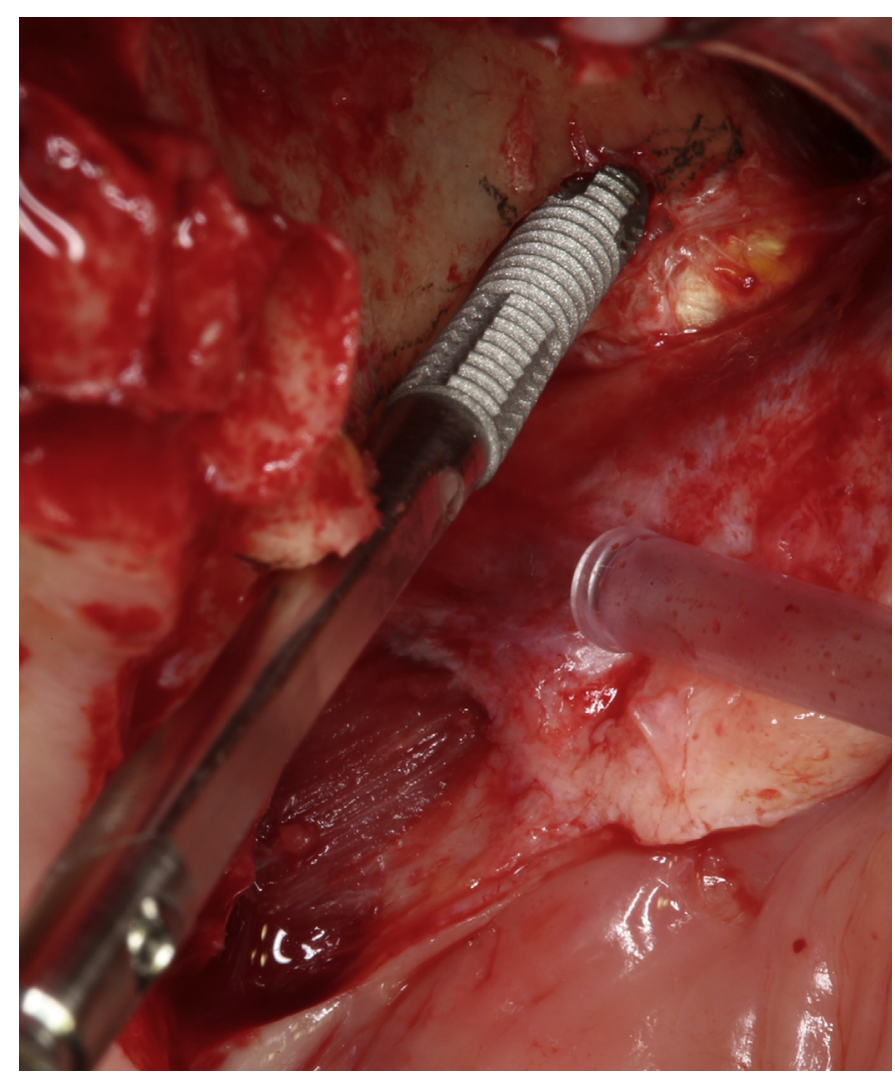

Fig. 35 Case 2: the unique tapered design feature on a $\mathrm{ZI}$ (Straumann ZAGA Flat), together with the use of a ZAGA conservative osteotomy, allows for an enhanced implant primary stability.

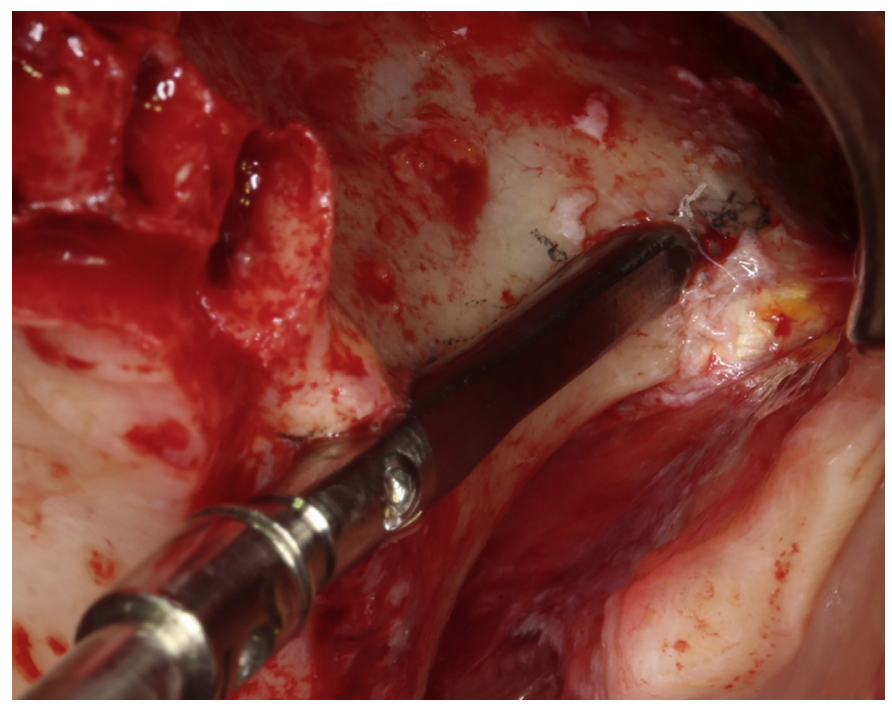

Fig. 36 Case 2: the implant (Straumann ZAGA Flat) and transport holder have the same diameter, helping the surgeon manage implant insertion in situations where the implant is inserted close to another implant or to hard bone.

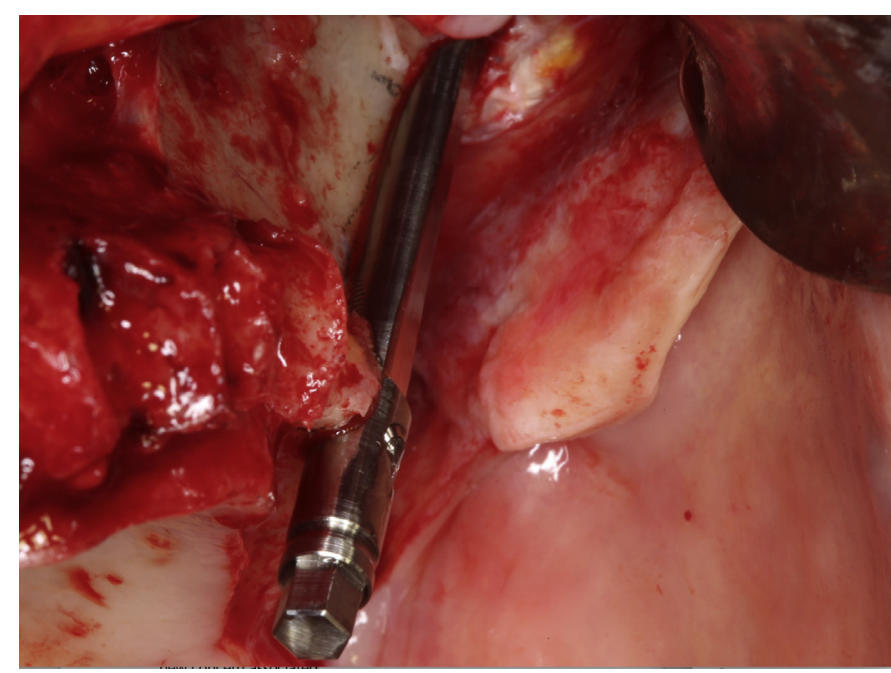

Fig. 37 Case 2: lateral view of the implant design (Straumann ZAGA Flat).

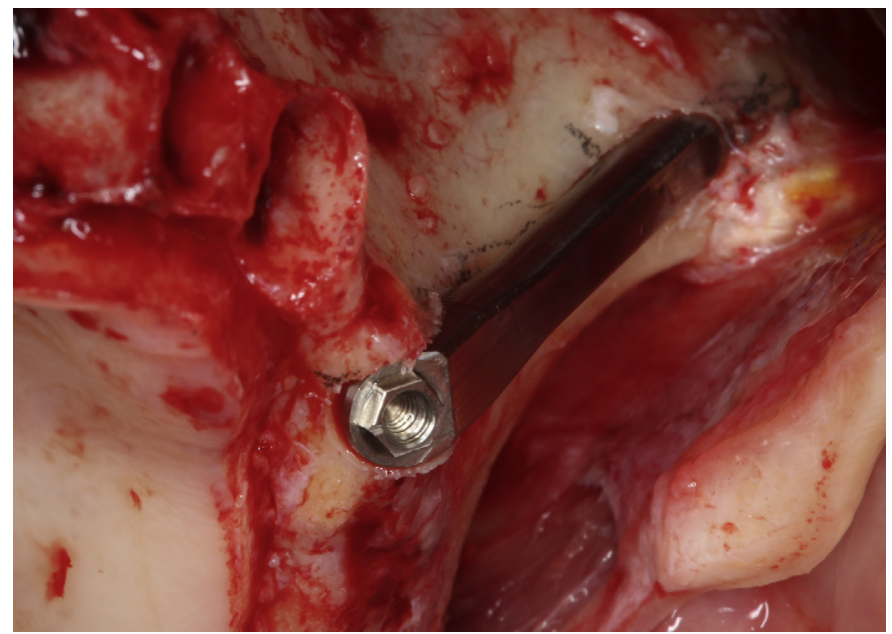

Fig. 38 Case 2: the implant in place is partially outside the bone envelope. To diminish eventual vascular compression of the soft tissues, the implant (Straumann ZAGA Flat) has a flat body section. 


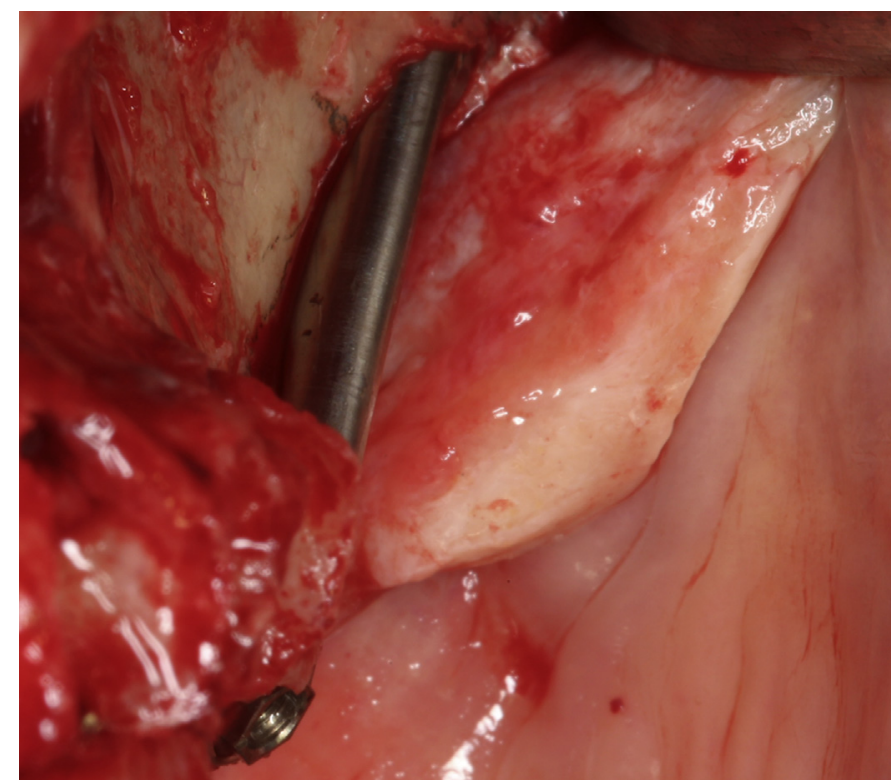

Fig. 39 Case 2: the Straumann ZAGA Flat implant from a lateral view. Its flat profile is not protruding from the remaining alveolar bone.

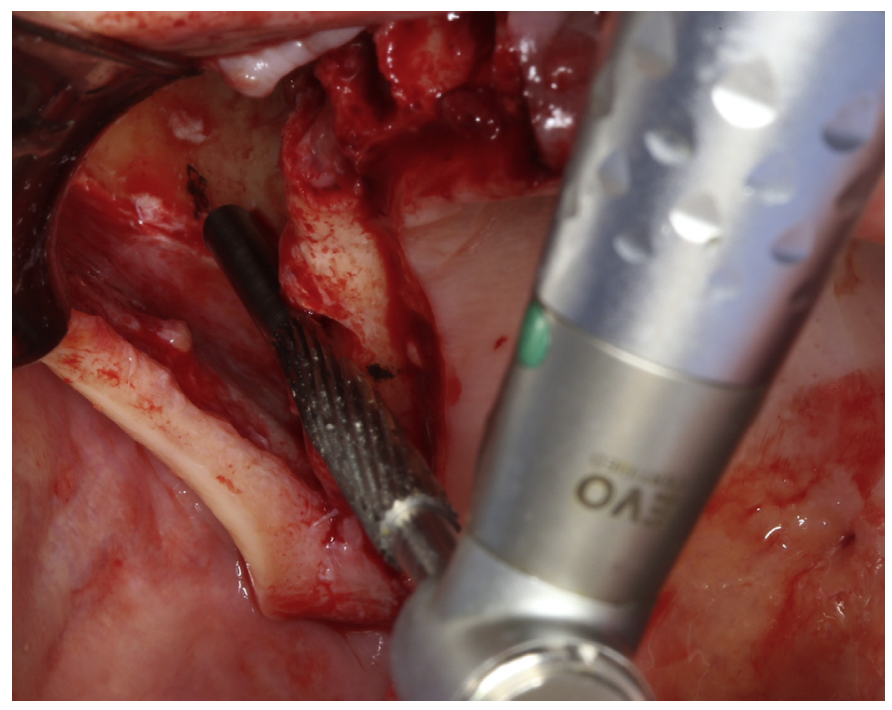

Fig. 40 Case 2: the Straumann AG lateral cutting bur is starting a channel osteotomy on the right posterior area.

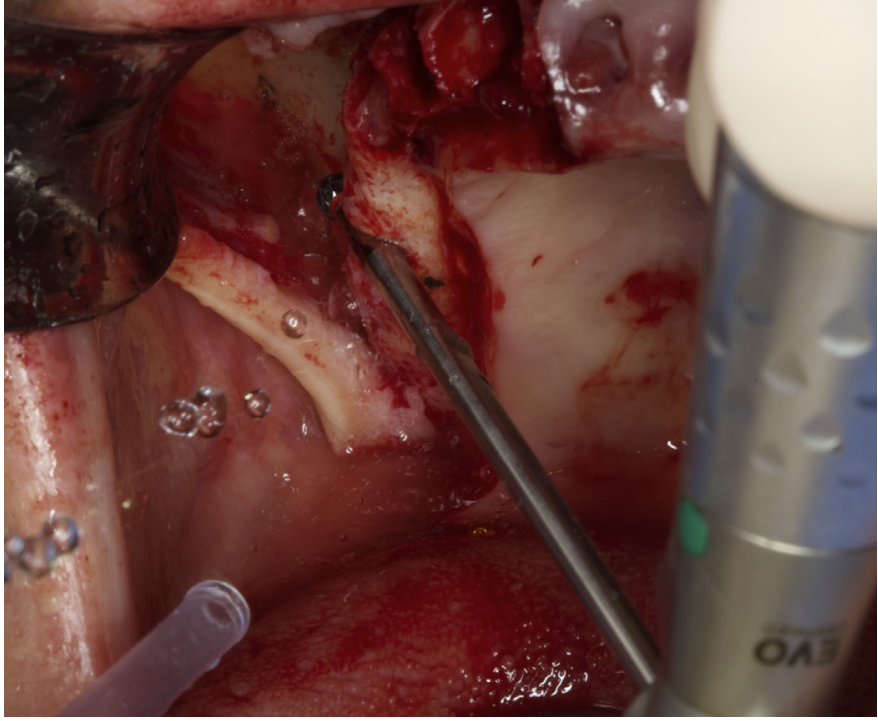

Fig. 41 Case 2: the initial alveolar channel is used for a smooth back and forward sliding movement of the round bur tail.

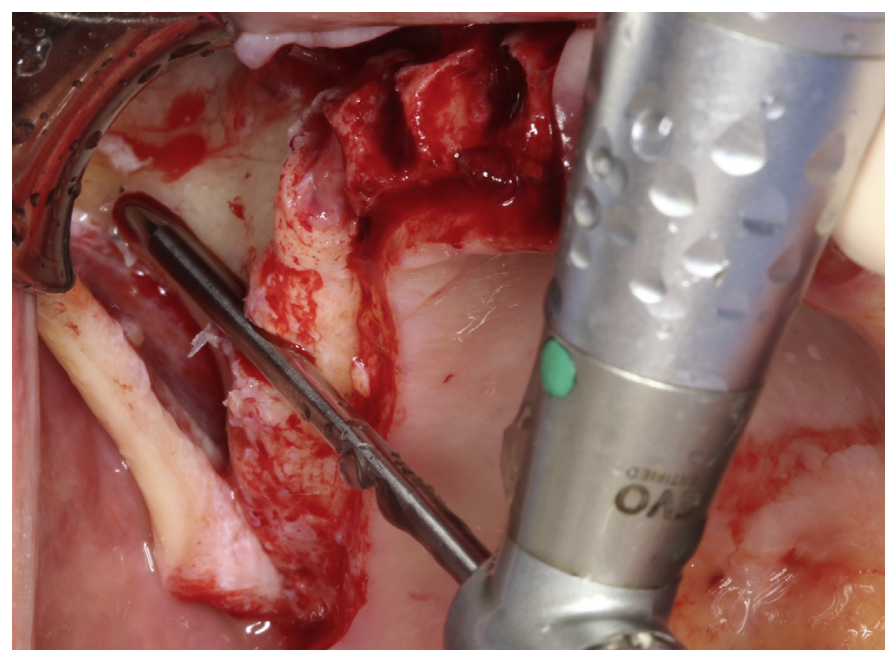

Fig. 42 Case 2: once the desired depth has been achieved, the round bur is perforating the maxillary buttress (antrostomy zone), with no care about sinus lining integrity. 


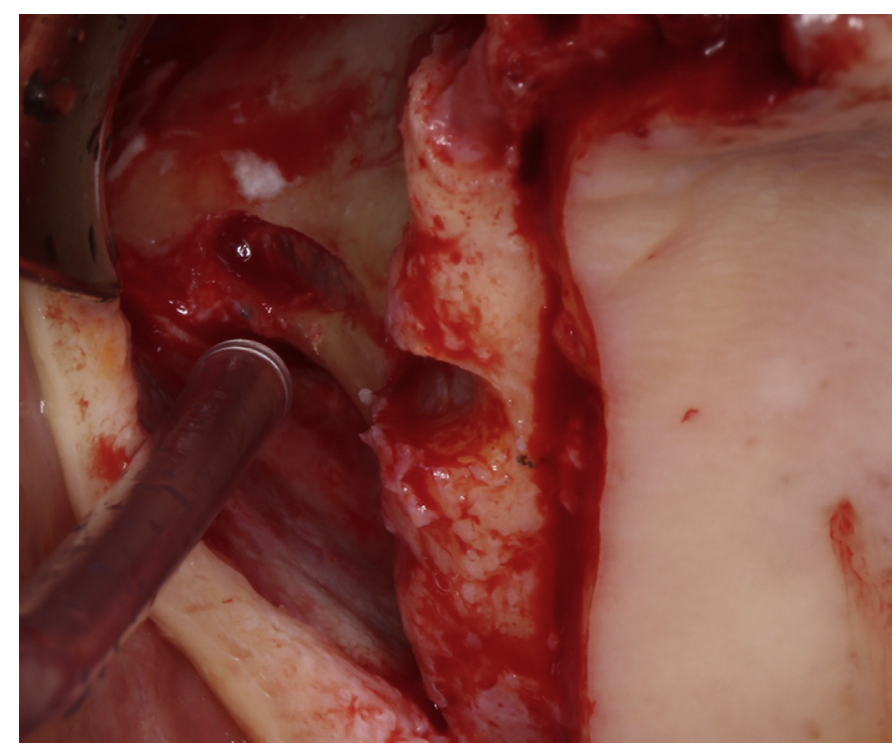

Fig. 43 Case 2: final ZAGA minimally invasive osteotomy achieved after the use of a narrow diameter twist drill. Note the integrity of the sinus membrane at the ZICZ.

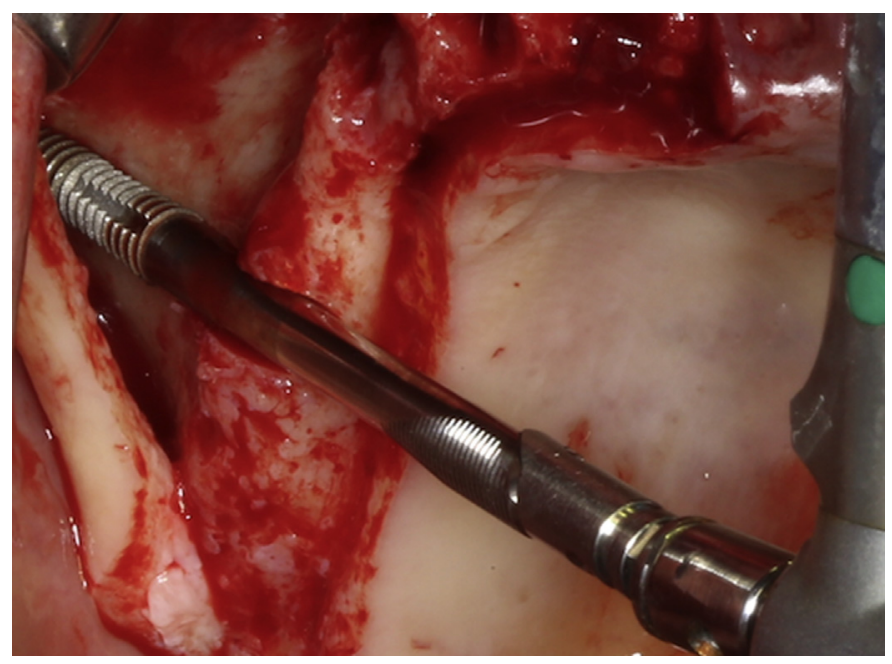

Fig. 44 Case 2: a Straumann ZAGA Flat $\mathrm{ZI}$ is being screwed on the zygomatic bone.

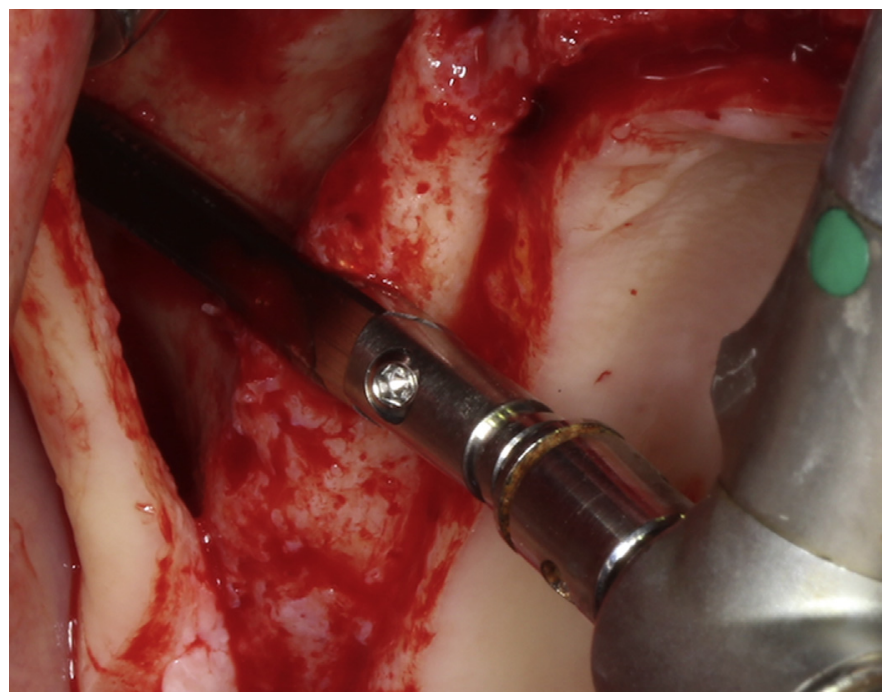

Fig. 46 Case 2: the implant mount of the ZAGA Flat design has the same diameter as the implant body. A $55^{\circ}$ pre-angled implant head has the potential for the use of straight abutments in almost all the situations.
Fig. 45 Case 2: neck detail of the Straumann ZAGA Flat ZI. Surface at the implant neck level opposite to the flat area, intended to be in intimate contact with the alveolar and maxillary wall bone, has been provided with mini threads. 


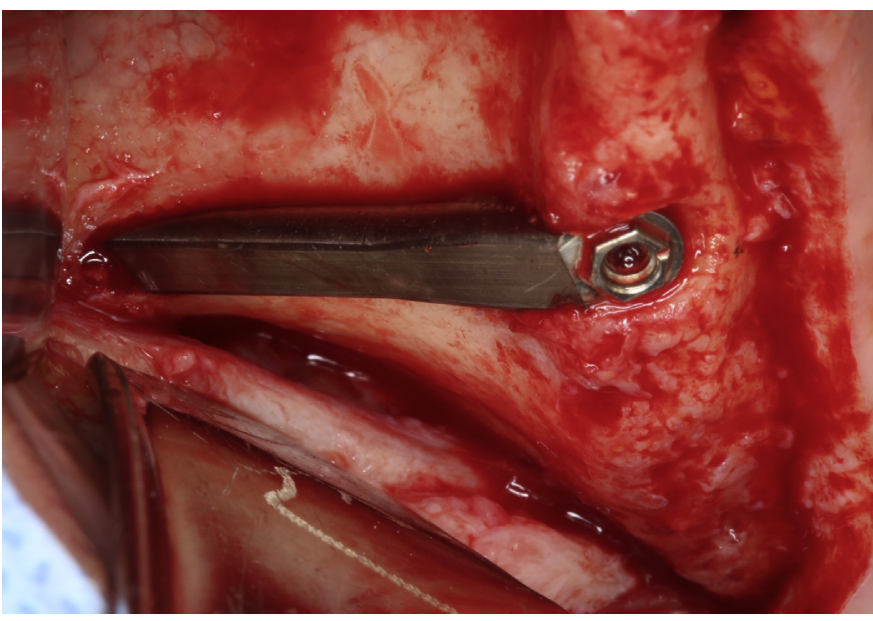

Fig. 47 Case 2: the ZAGA Flat implant design has been placed into a prosthetically driven position. Its section increases the surface facing the soft tissues, reducing an eventual implant pressure against them.

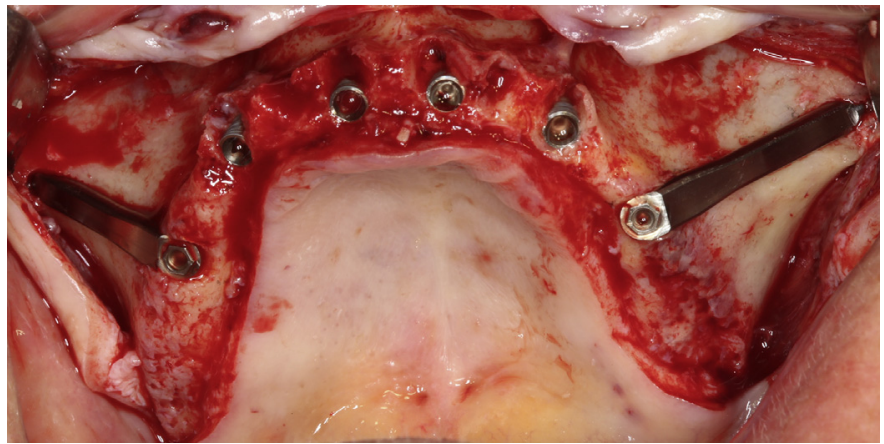

Fig. 49 Case 2: occlusal postoperative view. The ZAGA concept approach was used for obtaining both osteotomies in a minimally invasive manner.

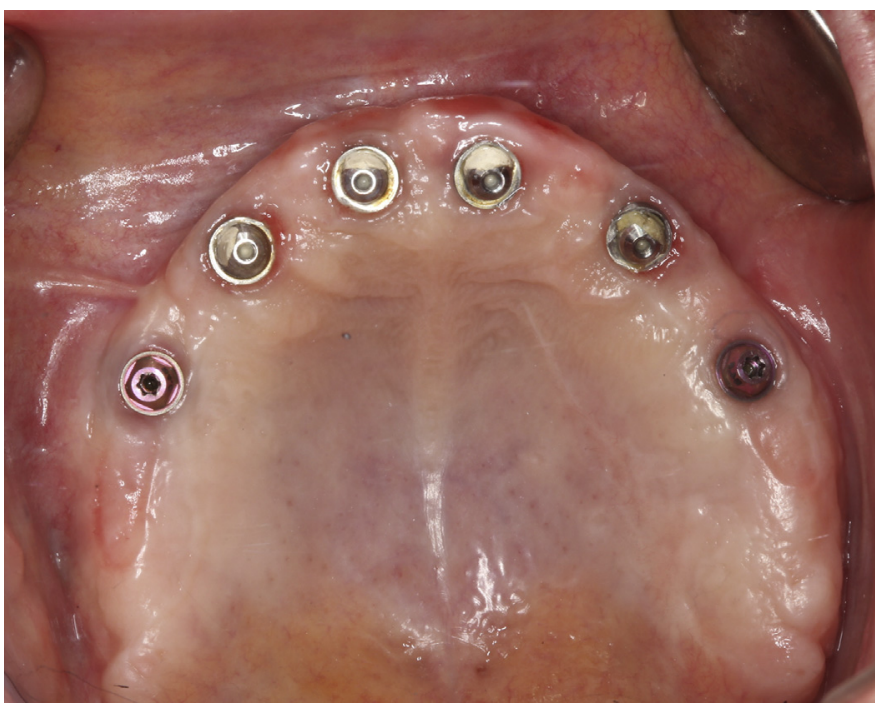

Fig. 51 Case 2: the 1-year control shows the results of combining the use of the ZAGA concept for hard and soft tissue management and an implant design with features adapted to the patient anatomy (Straumann ZAGA Flat).

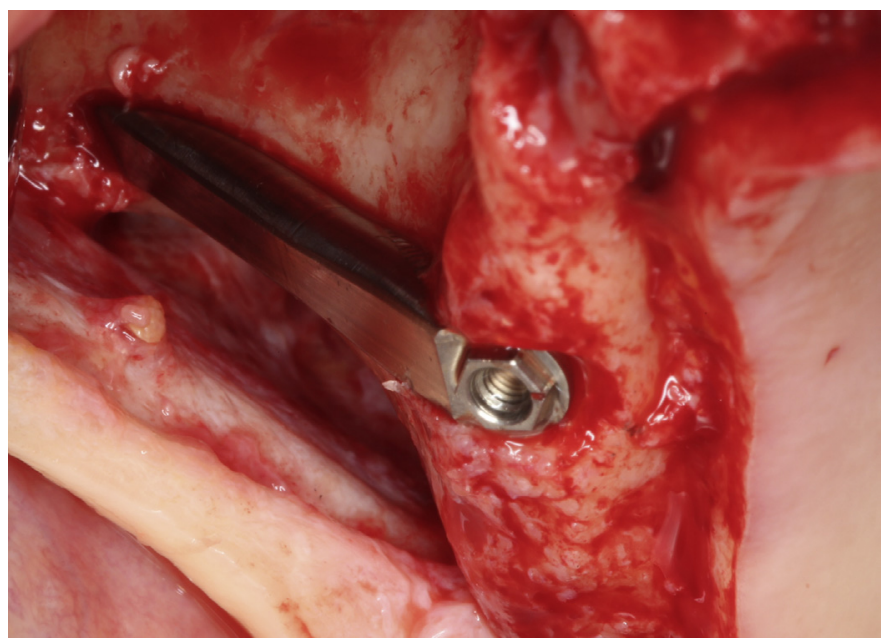

Fig. 48 Case 2: hexagonal $55^{\circ}$ pre-angled implant head (Straumann ZAGA Flat) placed in an ideal prosthetically guided position. Note the close contact between the implant and the remaining bone.

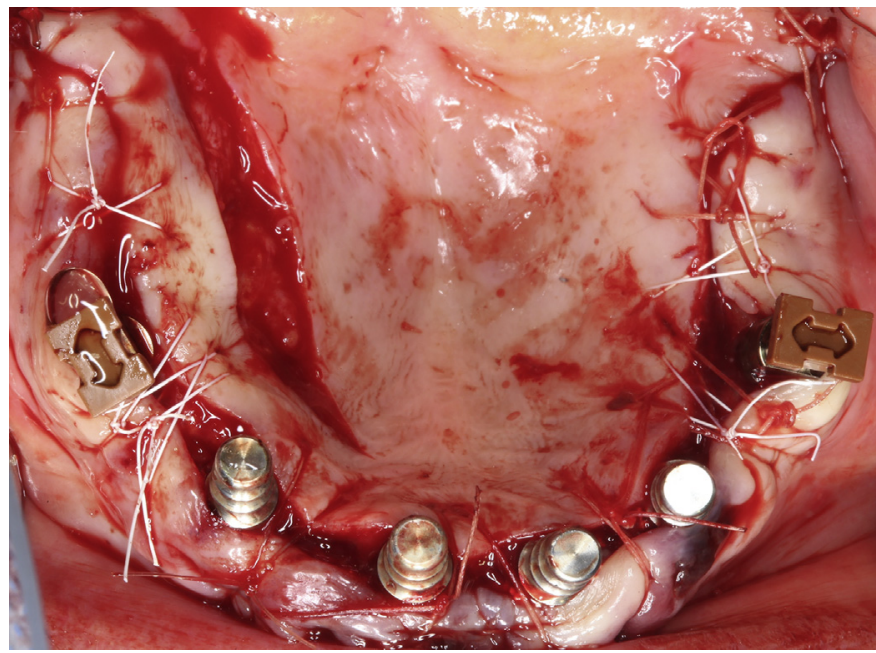

Fig. 50 Case 2: to maximize the amount of keratinized tissue buccal to the implants, a palatal incision was made. To facilitate a first intention closure, double pediculated partial flap was performed.

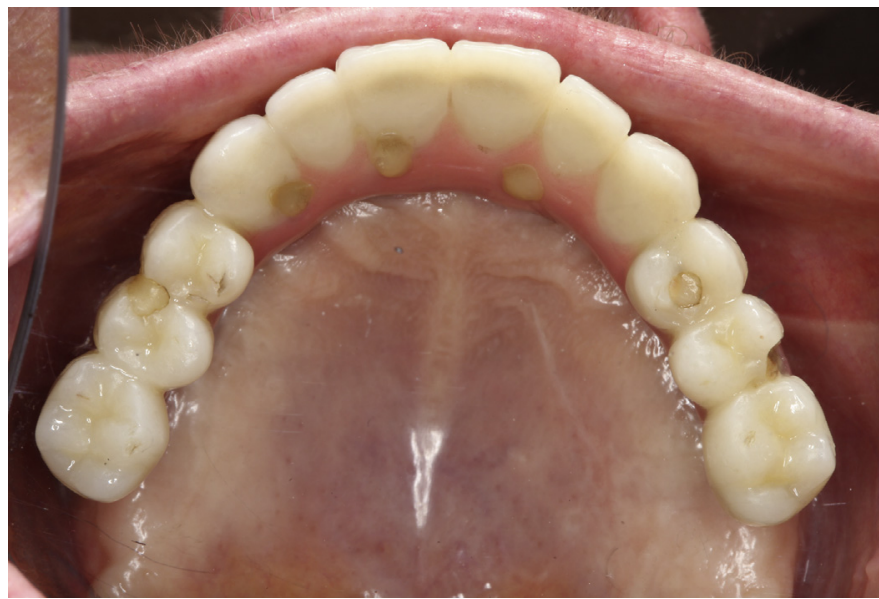

Fig. 52 Case 2: 1-year occlusal view of the prostheses placed by Drs Peter and Madalina Simon, ZAGA Center Stuttgart, Germany. Straight, non-angulated, Straumann abutments were used. 


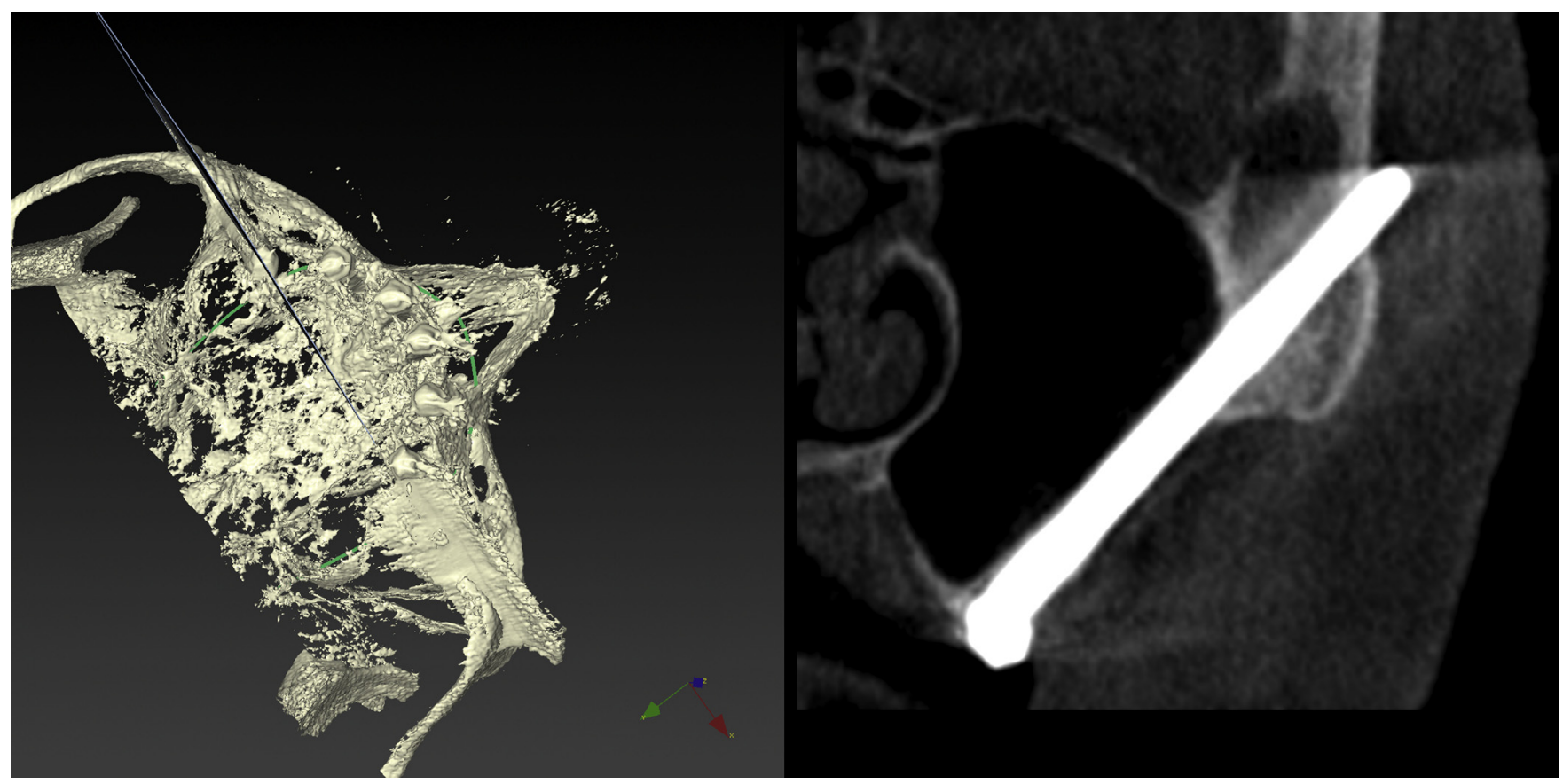

Fig. 53 Case 2: right side 3D and 2D images representing the anatomic features and sinus status of the final ZI trajectory at 1-year CBCT post-operative control. Compare with Fig. 22.

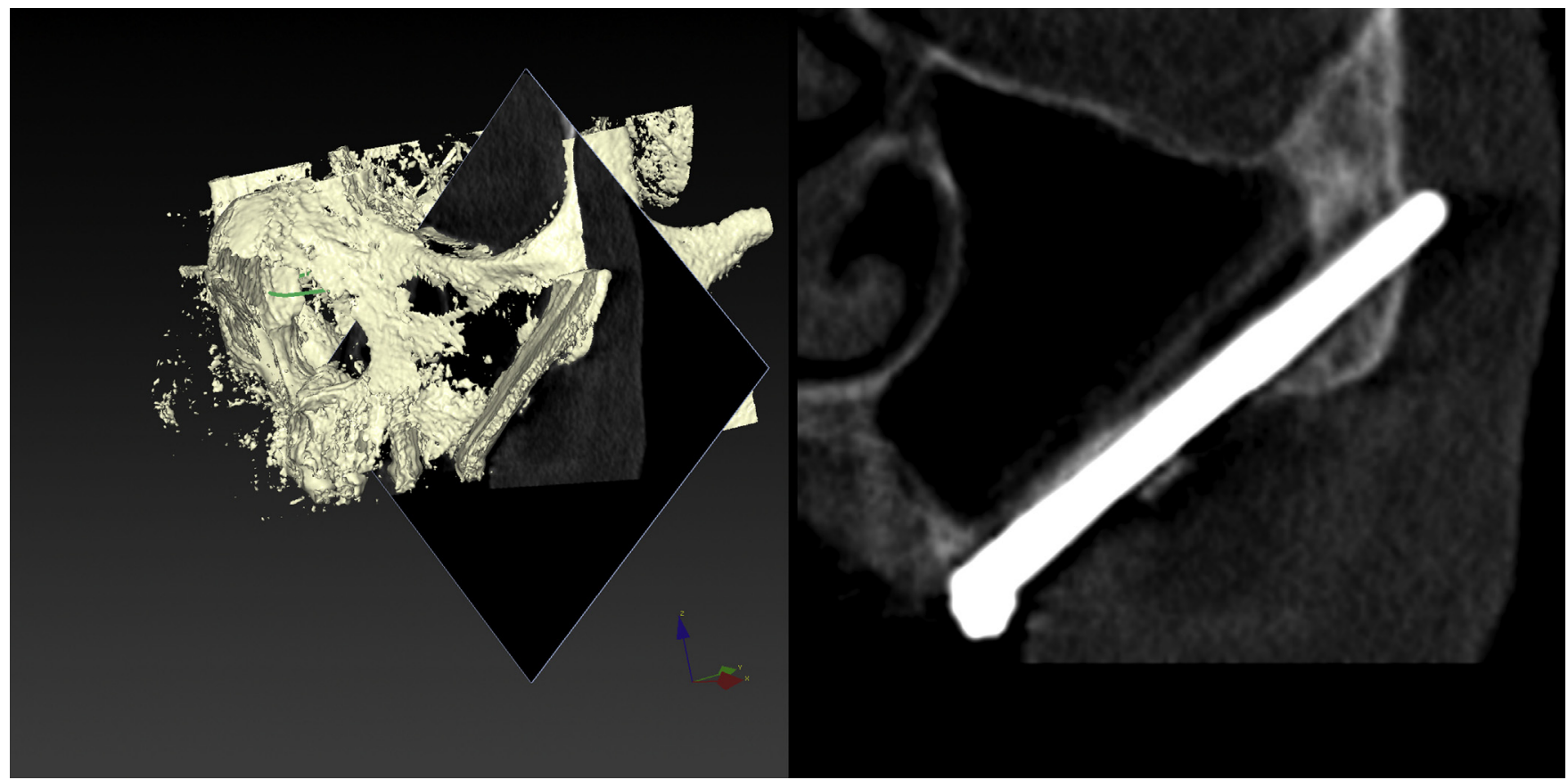

Fig. 54 Case 2: left side 3D and 2D images representing the anatomic features and sinus status of the final ZI trajectory at 1-year CBCT control. Compare with the preoperative status shown in Fig. 24. 

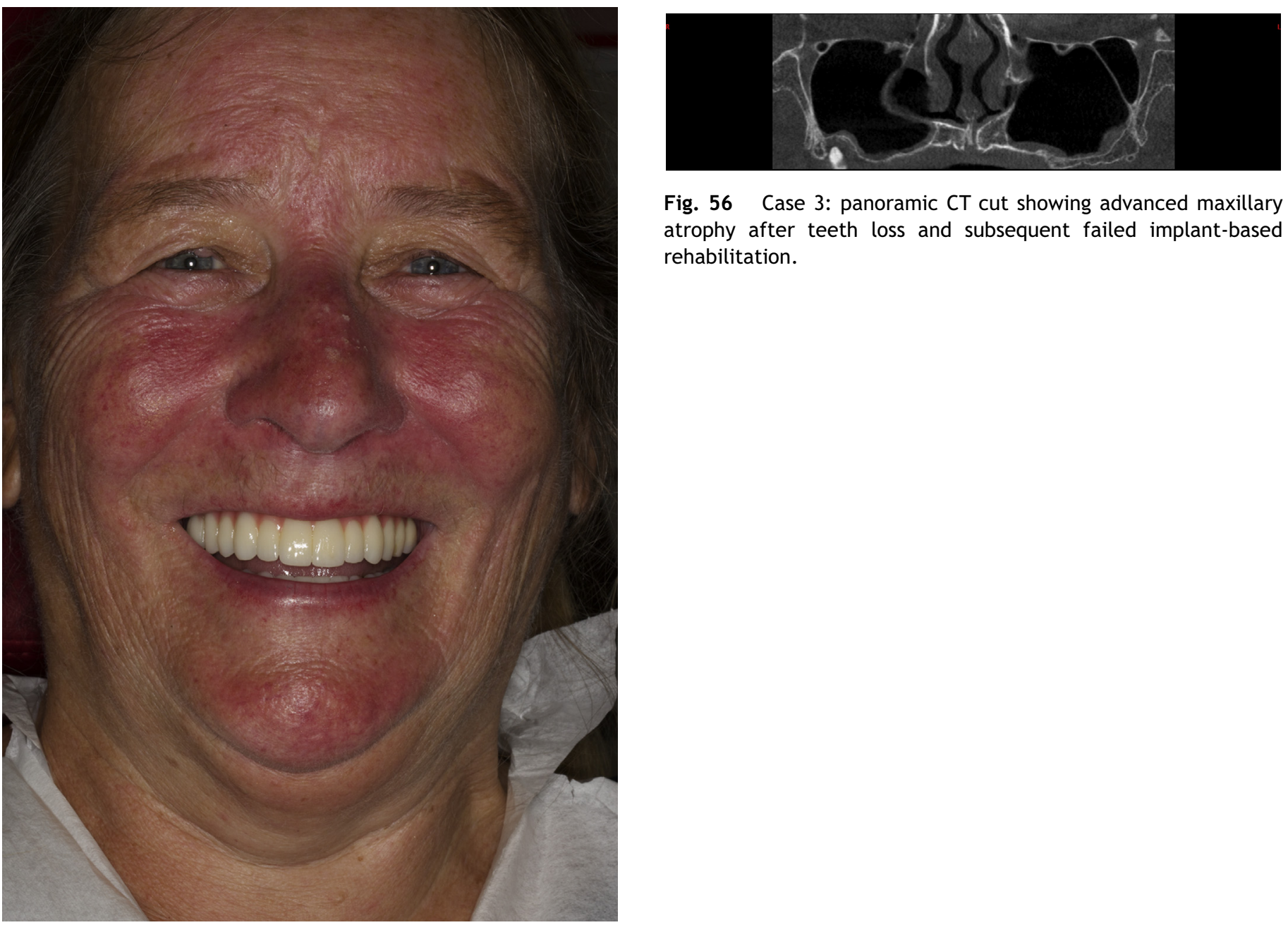

Fig. 56 Case 3: panoramic CT cut showing advanced maxillary atrophy after teeth loss and subsequent failed implant-based rehabilitation.

Fig. 55 Case 2: patient satisfaction is shown at 1-year control. Prostheses placed by Drs Peter and Madalina Simon, ZAGA Center Stuttgart, Germany.

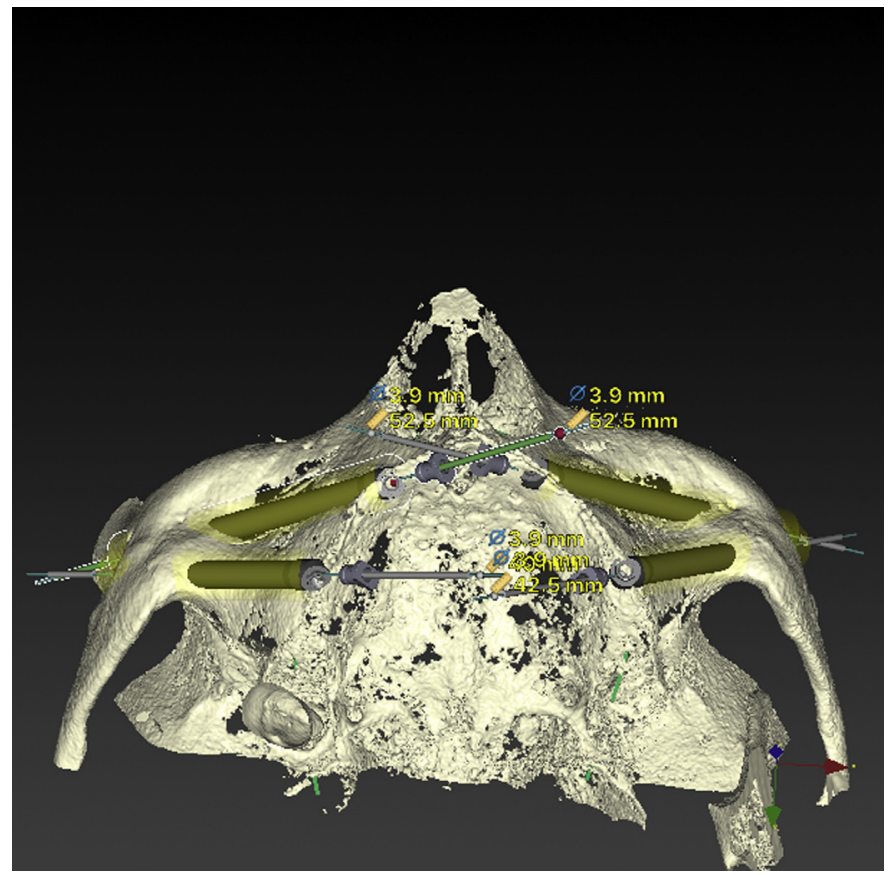

Fig. 57 Case 3: virtual planning. Anterior implants are foreseen at a position between lateral incisors and canines. Posterior implants would be located between the first molars and second premolars. 


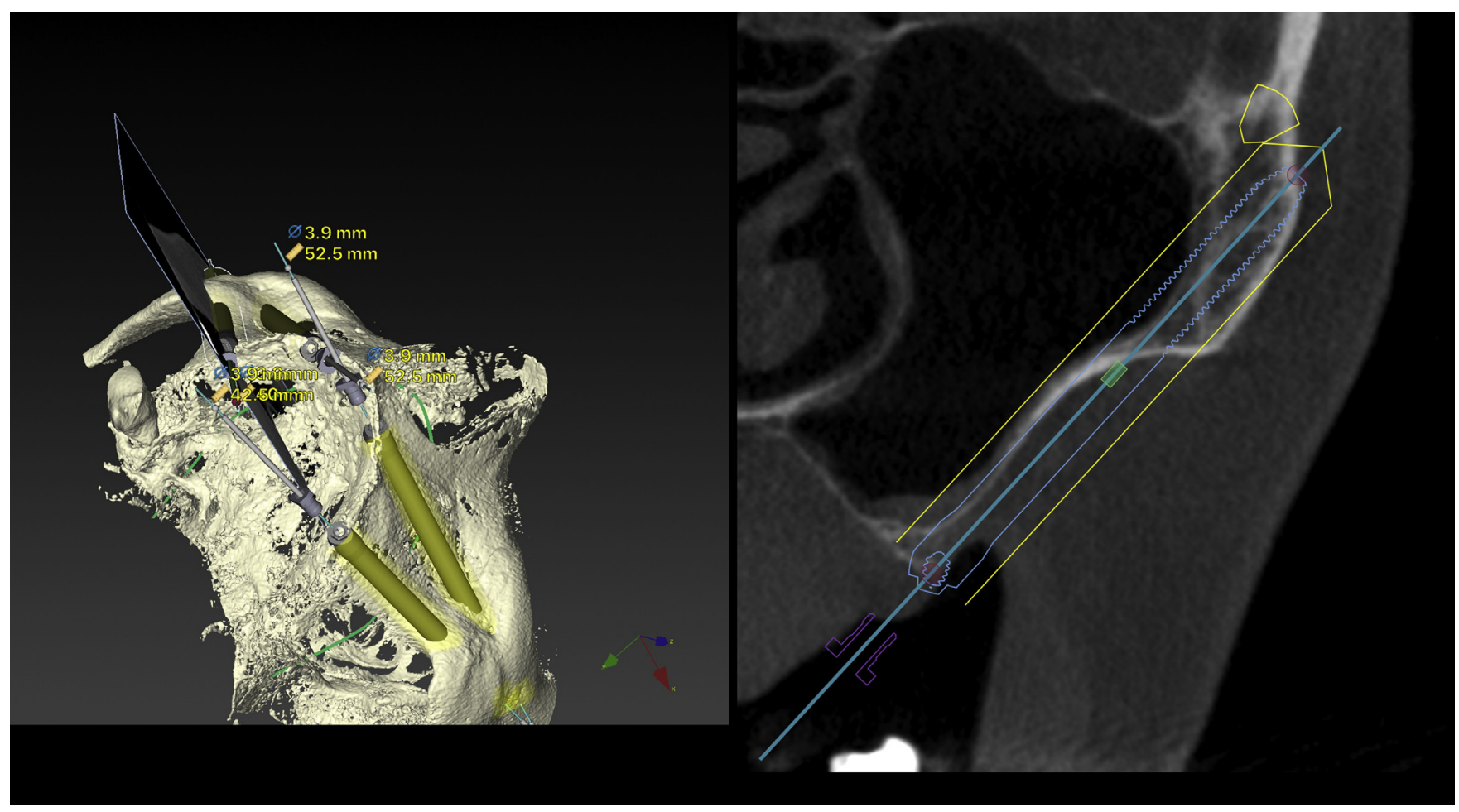

Fig. 58 Case 3: extrasinus planning in a ZAGA type 4 maxilla. The 3D and 2D images are representing the anatomic features of an eventual $\mathrm{ZI}$ trajectory in the position of the first upper right molar.

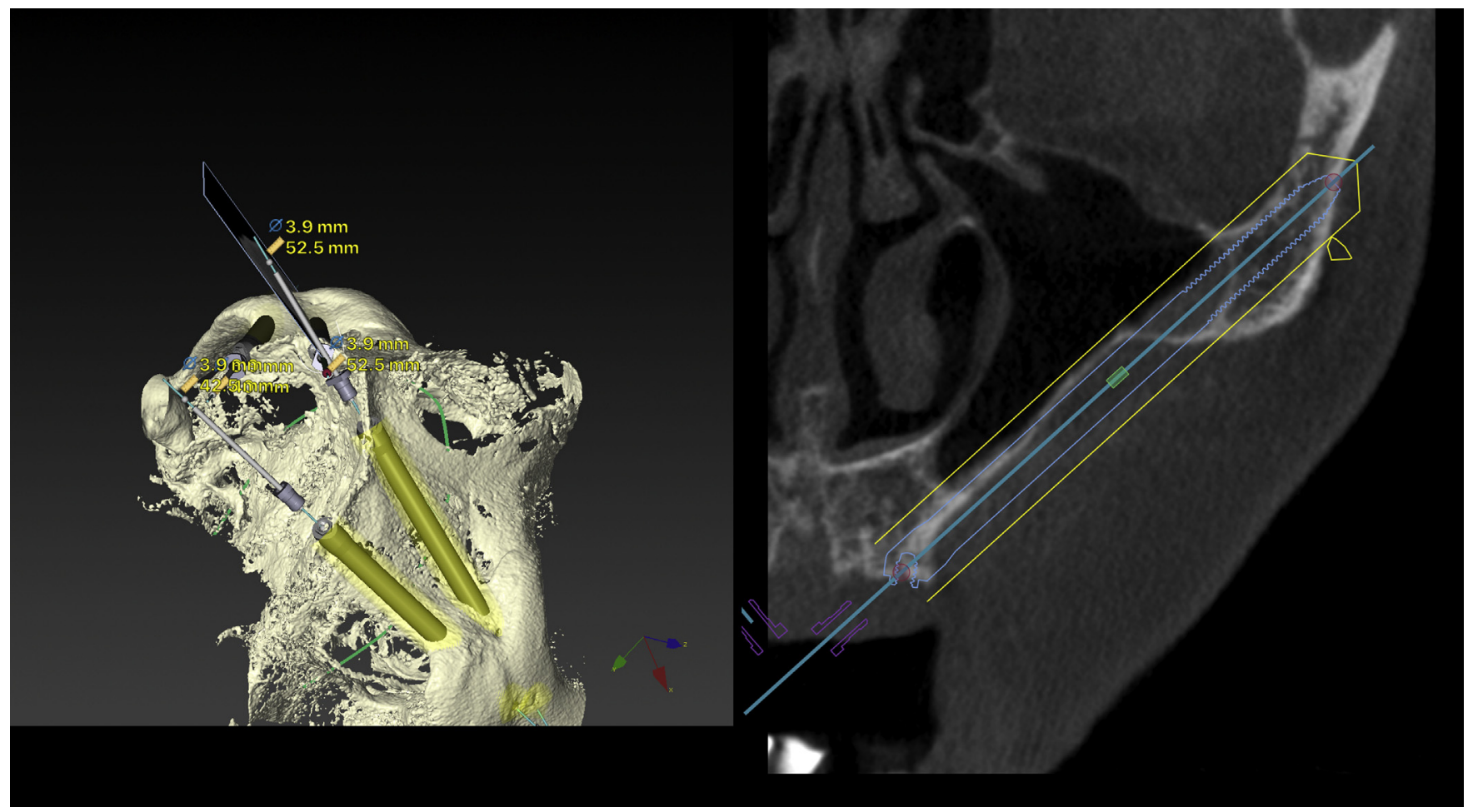

Fig. 59 Case 3: ZAGA type 3 maxilla, a tunnel-type osteotomy for the anterior right implant was planned. 


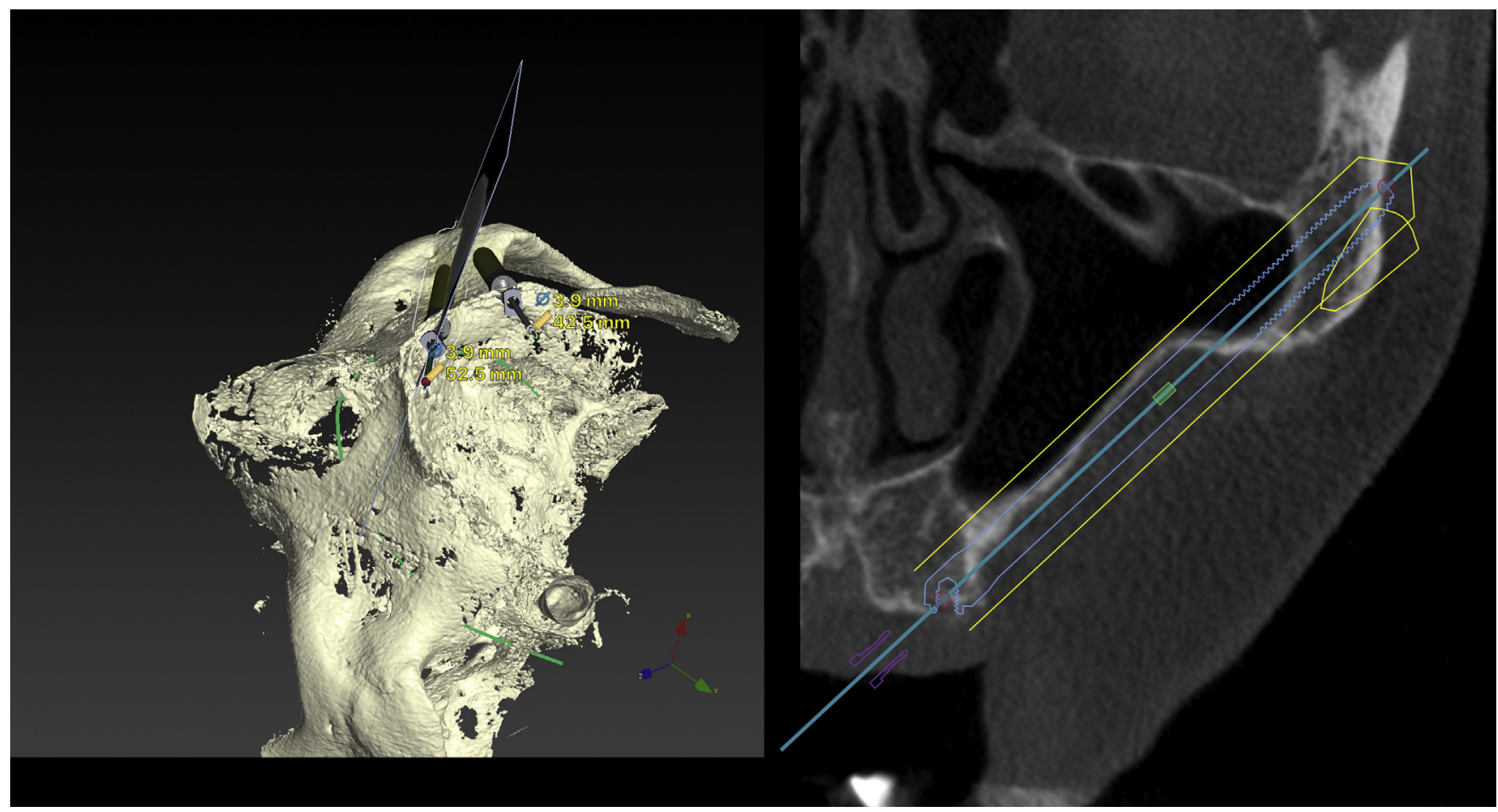

Fig. 60 Case 3: 3D and 2D images illustrating the anatomic features of an eventual ZI trajectory inthe aproximate position of the upper left canine. Maxilla anatomy is in between types 2 and 3, a tunnel-type osteotomy was planned.

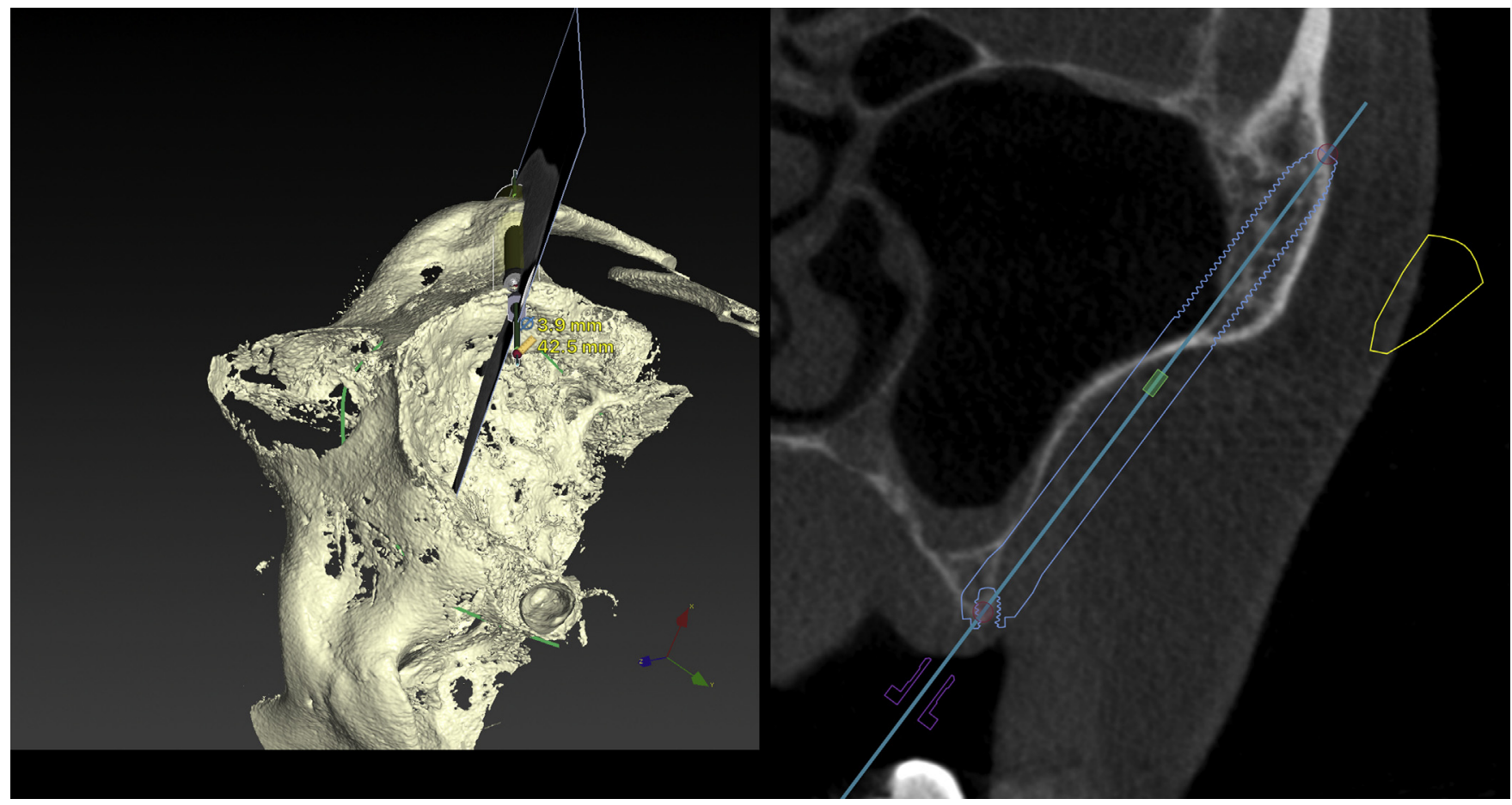

Fig. 61 Case 3: planning of posterior left implant. Although being a type 4 maxilla, the ZAGA channel-type osteotomy together with appropriate implant design eventually would be enough to host the implant section inside the alveolar bone. 


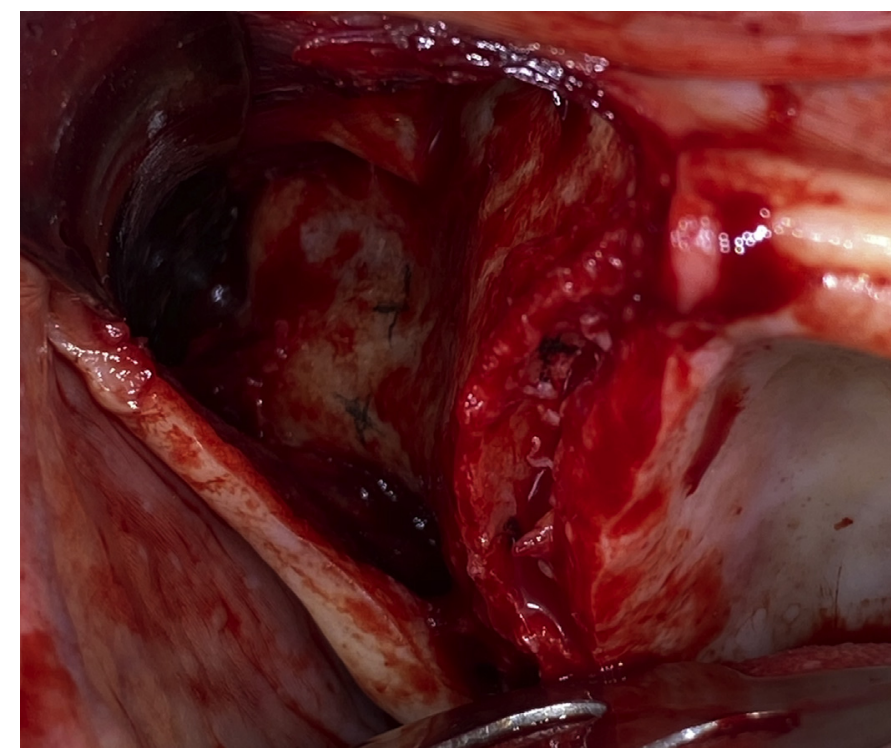

Fig. 62 Case 3: previous to drilling it is advisable marking the entrance points both at alveolar and zygomatic levels.

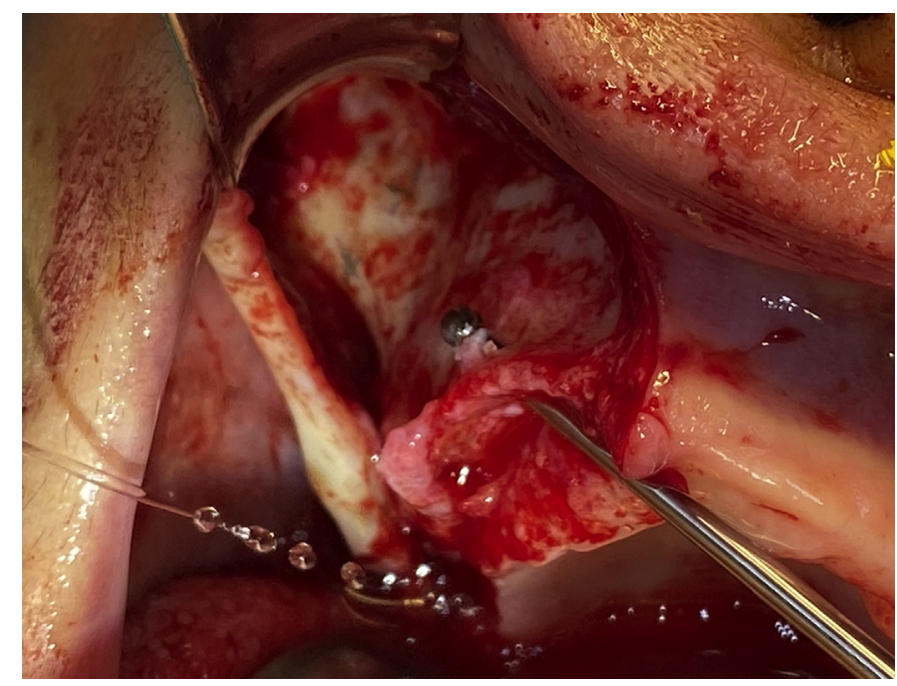

Fig. 64 Case 3: the bur direction is corrected progressively until reaching the marked apical point.

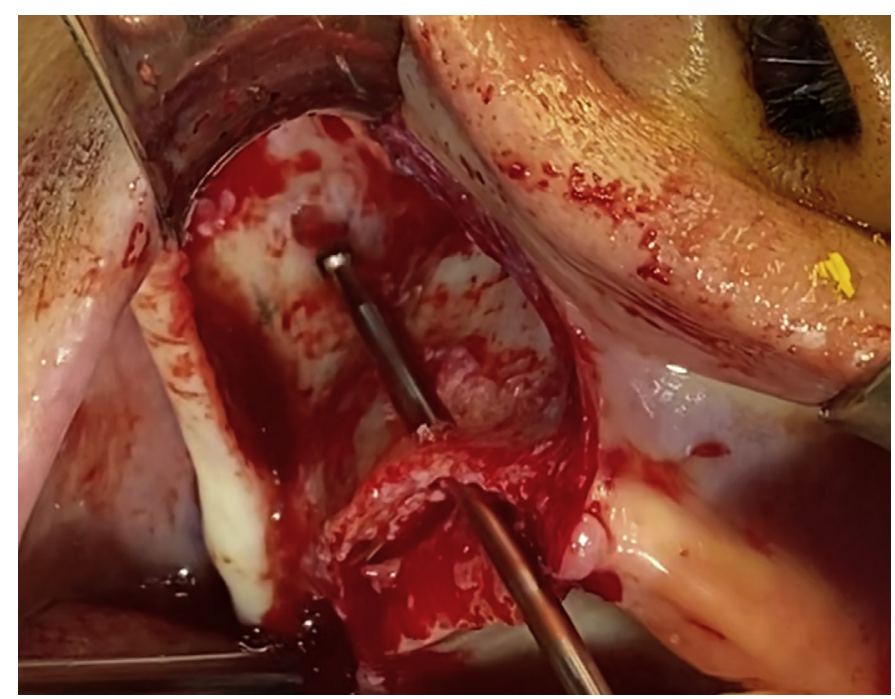

Fig. 66 Case 3: the round bur tail is smoothly sliding using the channel support; the bur direction is maintained during the movement.

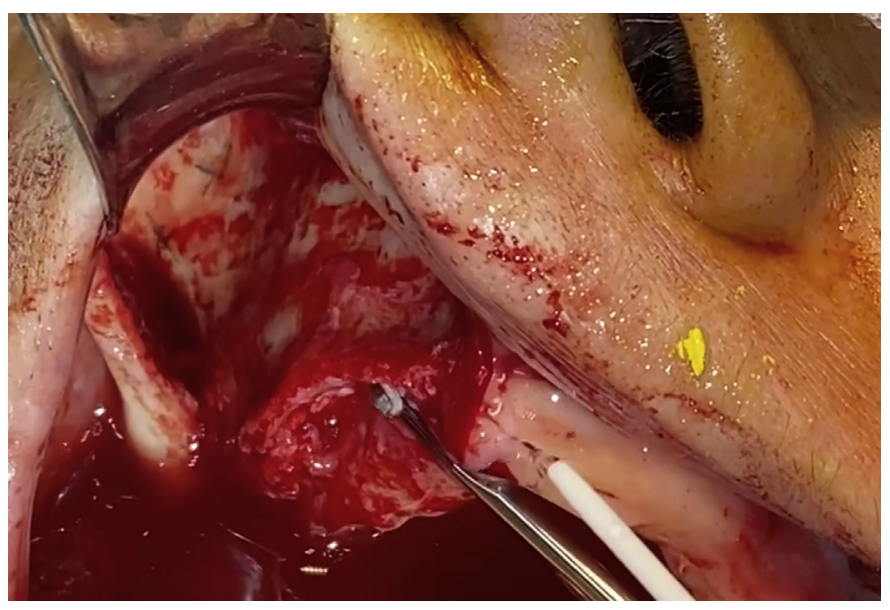

Fig. 63 Case 3: alveolar bone height is moderate; the round bur would point buccally in to avoid nose or sinus perforation. Direction will be corrected later.

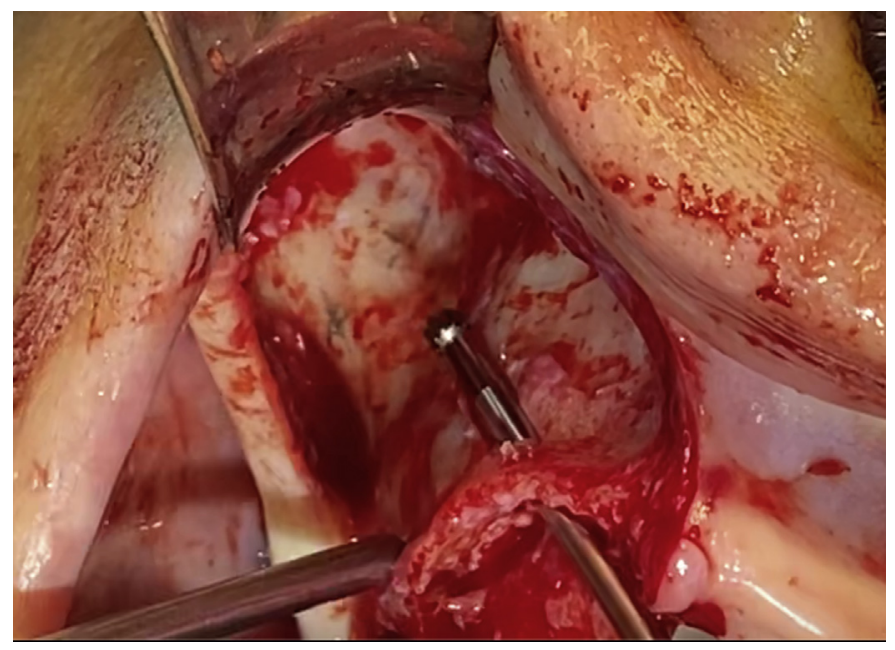

Fig. 65 Case 3: a channel was carved on the maxillary wall. In order to maintain sinus membrane integrity, the round bur will cut bone just on its back movement.

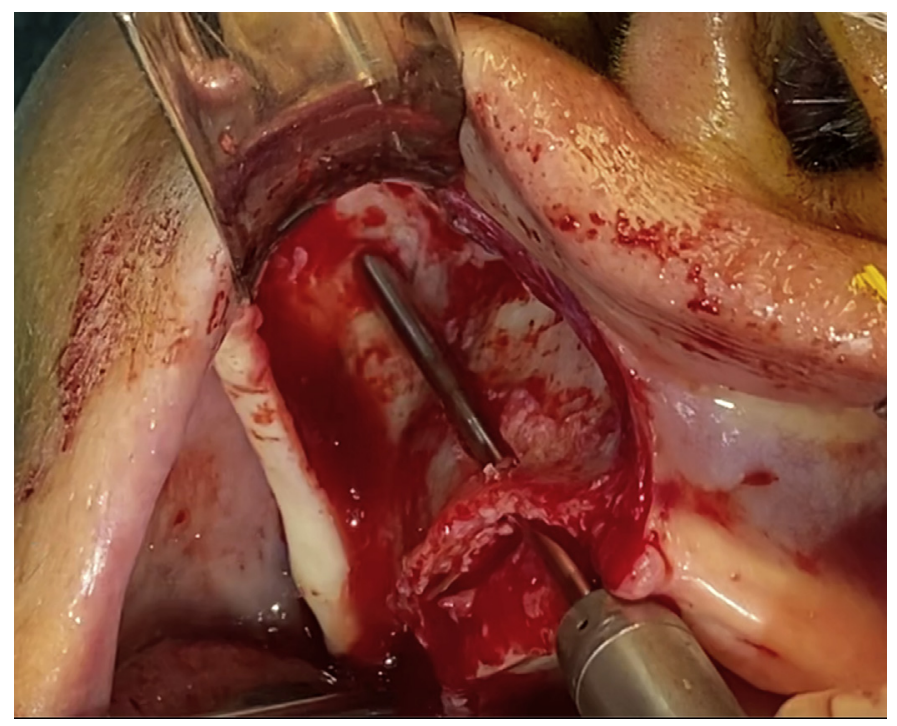

Fig. 67 Case 3: the round bur has perforated the zygomatic process of the maxilla. No care is taken for sinus lining integrity at this level. 


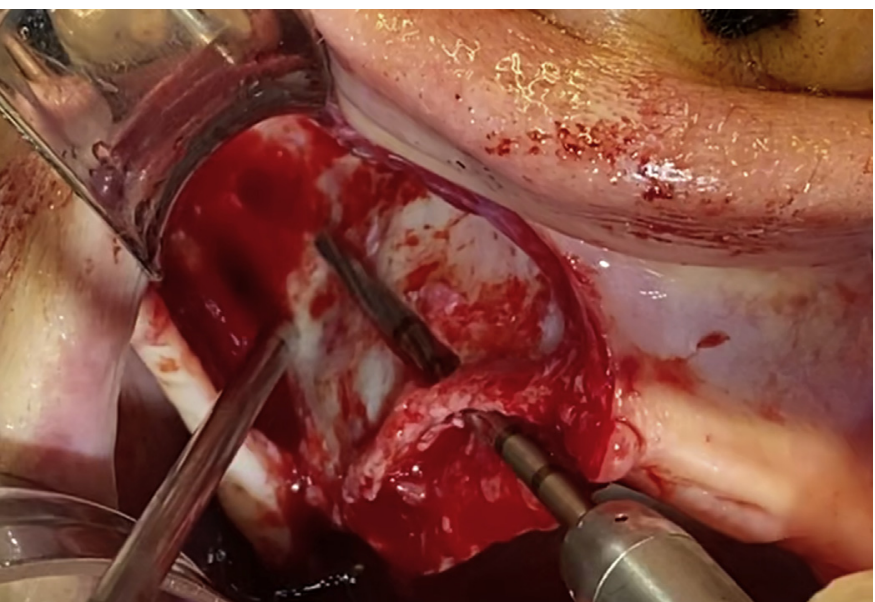

Fig. 68 Case 3: optionally, the lateral cutting properties of a zygo-bur (Versah.com) are used for ultimate smoothing and enlarging of the implant path.

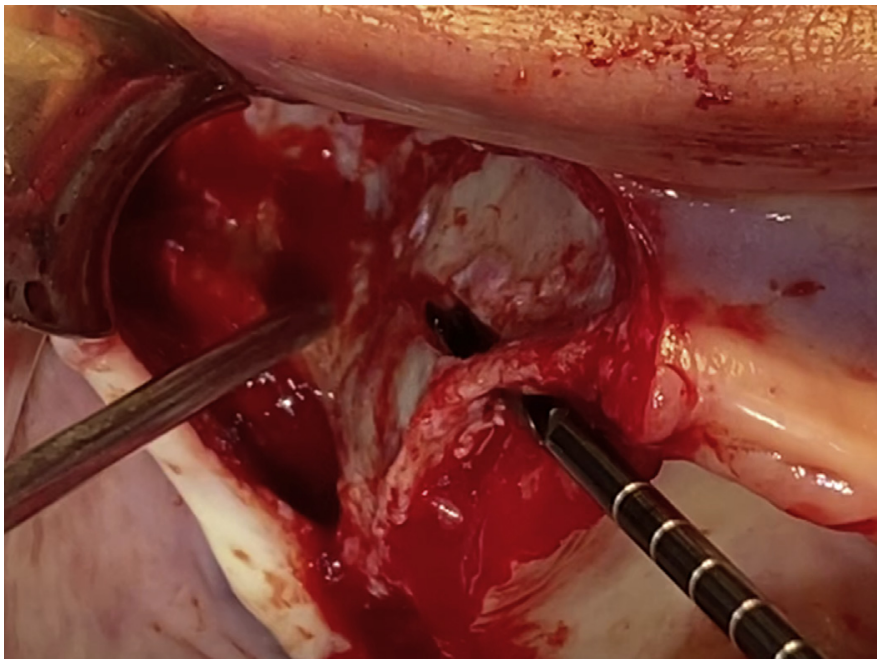

Fig. 70 Case 3: when poor zygomatic bone quality or quality are present it is appropriate to diminish final drill diameter from the regular 2.9-mm (Straumann AG) until 2.8- $\mathrm{mm}$ or even $2.7-\mathrm{mm}$ of diameter. A stepped twist drill of $2.8-\mathrm{mm}$ diameter was elected as the final drill.

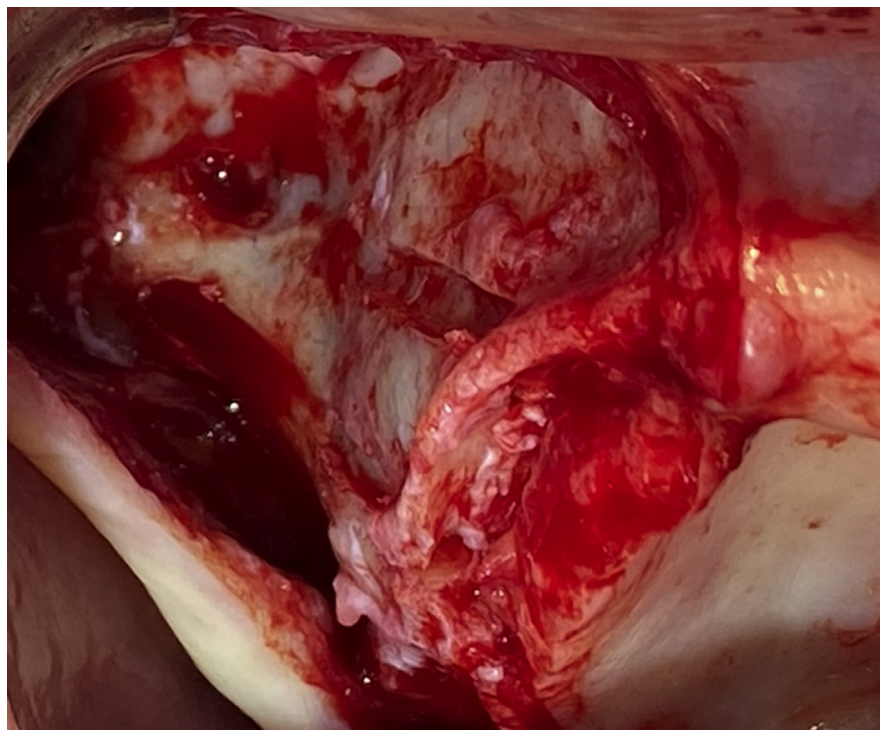

Fig. 72 Case 3: a conservative, minimally invasive osteotomy has been achieved. Sinus integrity has been maintained.

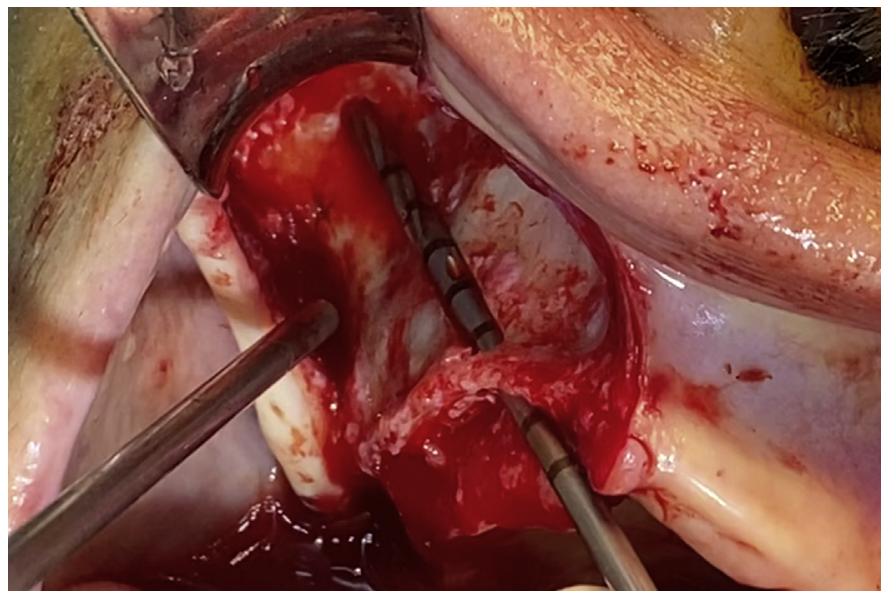

Fig. 69 Case 3: the zygo-bur (Versah.com) is used in clockwise direction to achieve a smooth tunnel into the zygomatic bone that guarantees no deviation for the next twist drill.

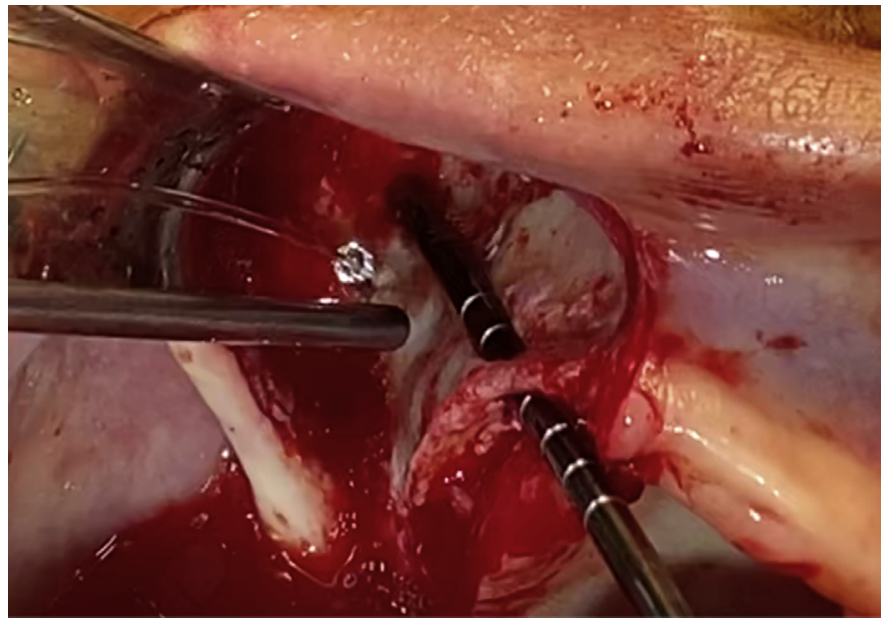

Fig. 71 Case 3: the stepped 2.8-mm diameter twist drill was used until thorough perforation of the zygomatic bone. A retractor previously placed on the angle formed by the frontal and temporal apophysis of the zygomatic bone would protect facial skin from eventual drill injury.

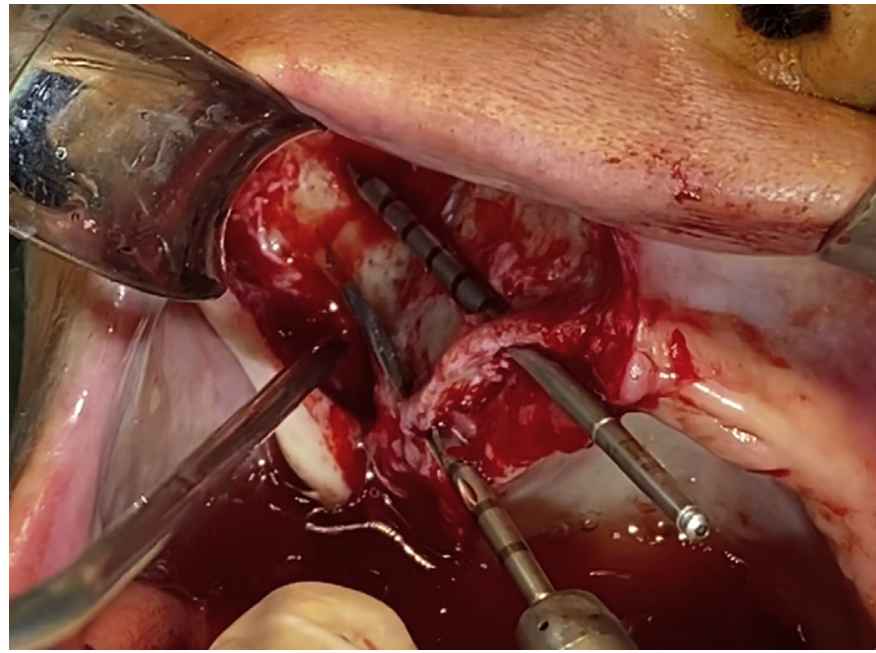

Fig. 73 Case 3: the zygo-bur is maintained on the anterior osteotomy as a guide while a shorter bur (versah.com) is used to start the ZAGA channel osteotomy for the posterior implant. 


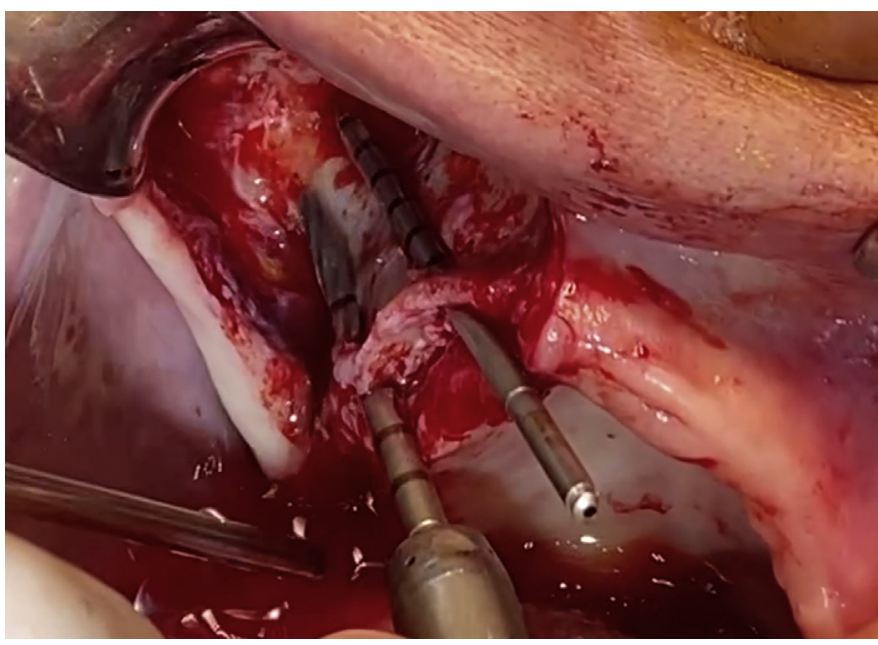

Fig. 74 Case 3: once adequate channel direction and depth have been achieved; a double, lateral and frontal cutting bur (versah. com) is used in a clockwise direction for antrostomy initiation.

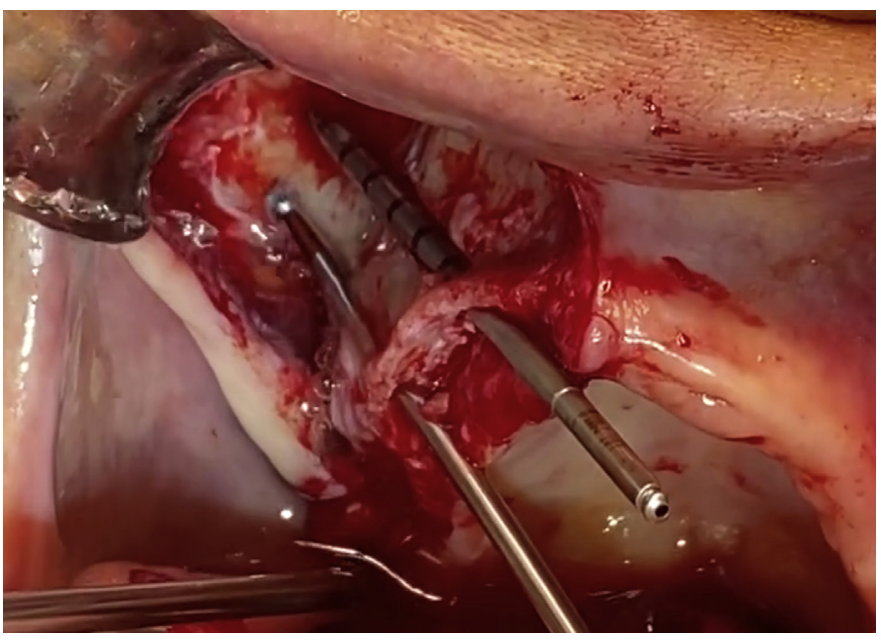

Fig. 76 Case 3: commonly, a zygomatic bony step is created on the facial side of the zygoma during the antrostomy. The round bur then is used for step beveling, so that the next drill direction will not be modified.

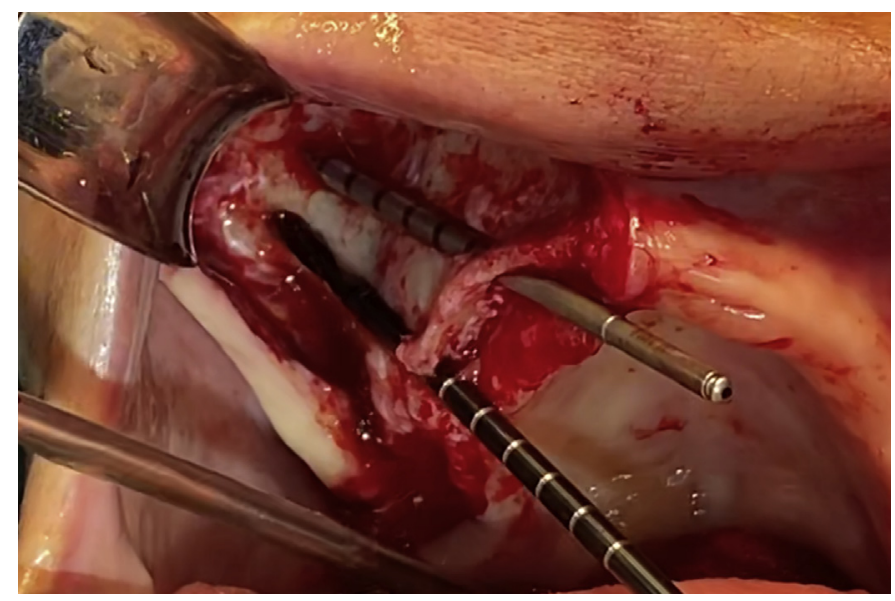

Fig. 78 Case 3: a 2.8-mm diameter twist drill was used as the final drill.

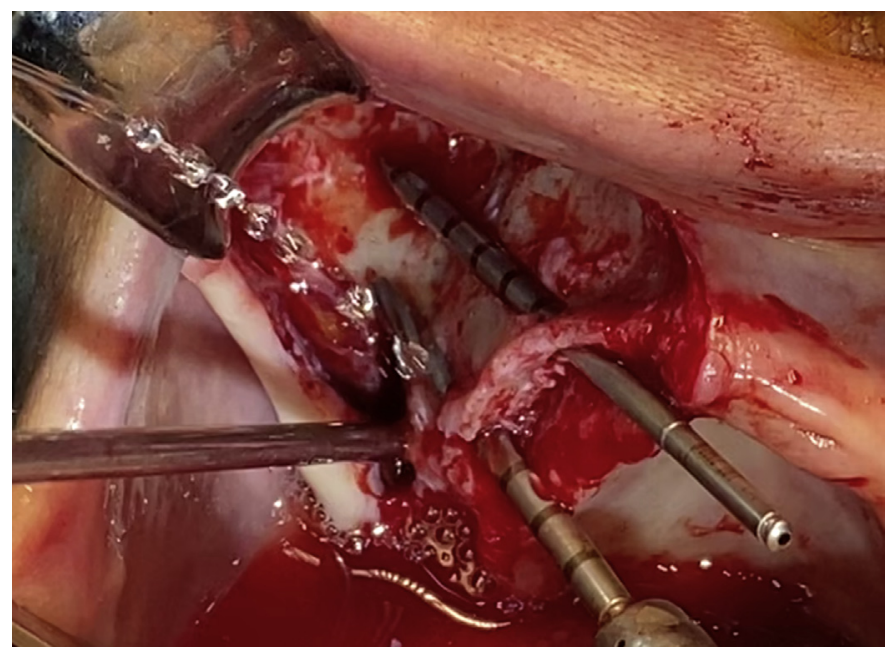

Fig. 75 Case 3: te lateral cutting power of the zygo-bur (versah. com) contributes to the achievement of a smooth channel where the next bur will slide maintaining its direction.

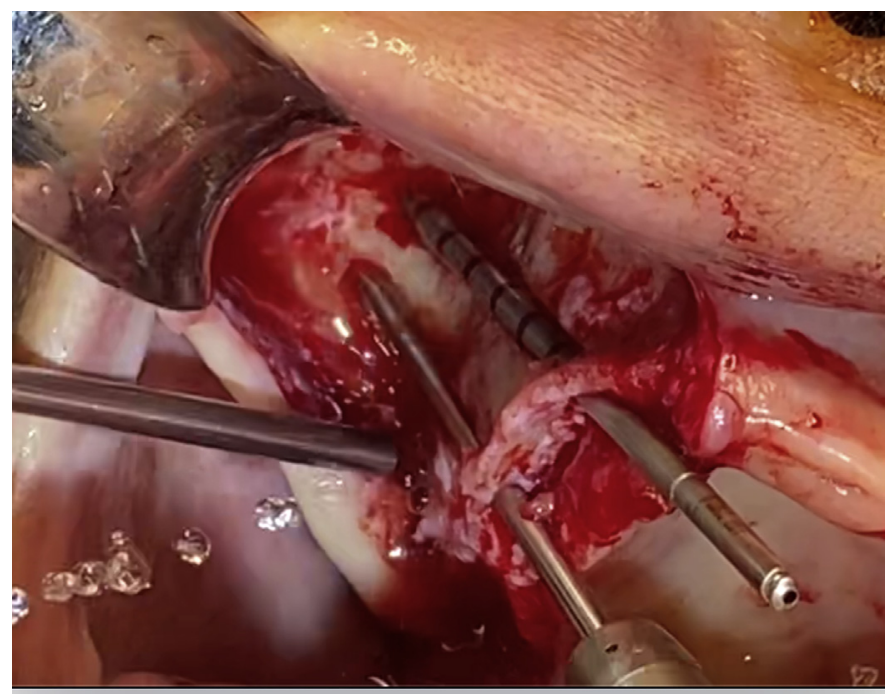

Fig. 77 Case 3: round bur is introduced into the zygomatic bone to facilitate maintenance on the achieved direction by the next drill.

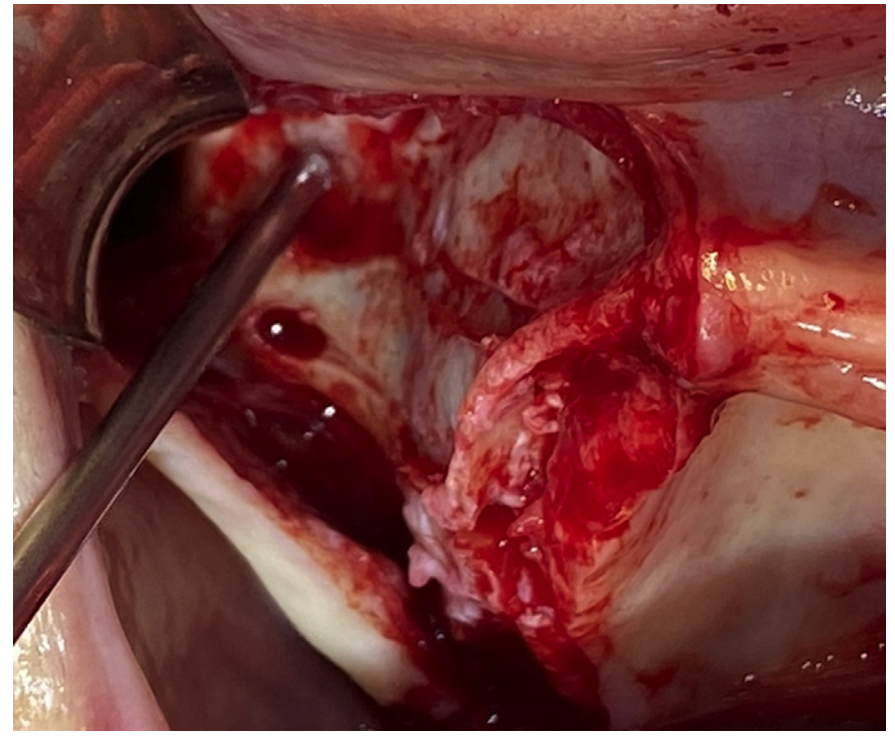

Fig. 79 Case 3: osteotomies of the right side have been performed. Remains of alveolar bone and maxillary wall have been respected. Sinus lining integrity at the ZICZ was maintained. 


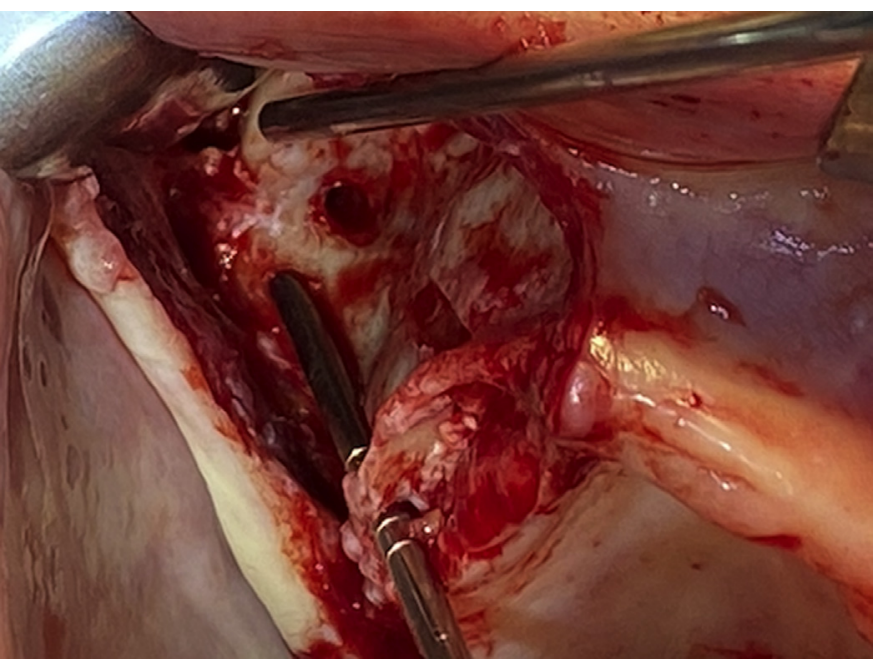

Fig. 80 Case 3: after measurement, distal implant will be placed first.

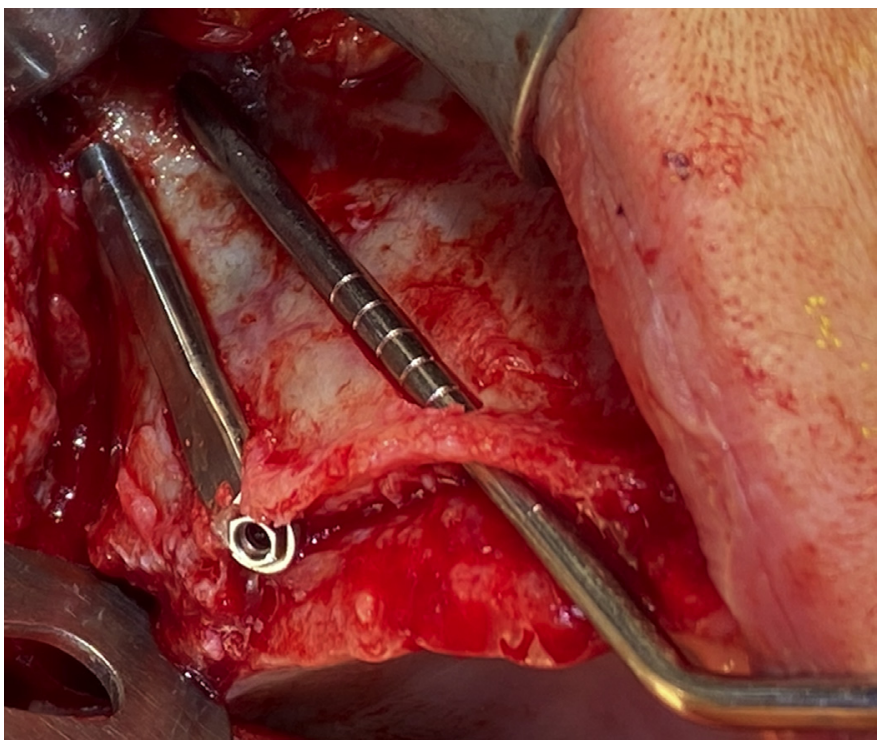

Fig. 82 Case 3: the anterior osteotomy is measured to double check the posterior implant is not crossing the anterior way.

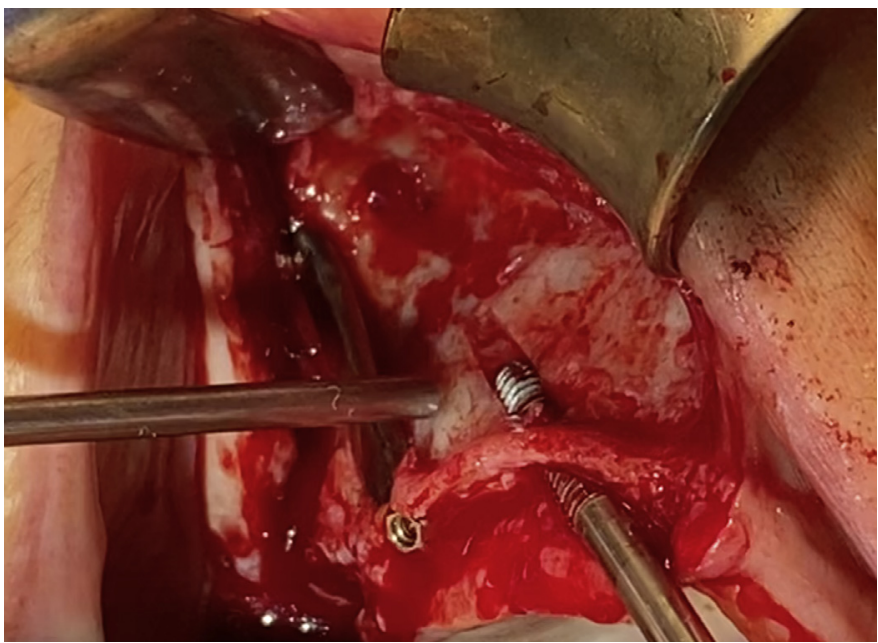

Fig. 84 Case 3: low-speed rotation of the implant (Straumann ZAGA Round) allows apical flutes for self-tapping effect on the alveolar bone.

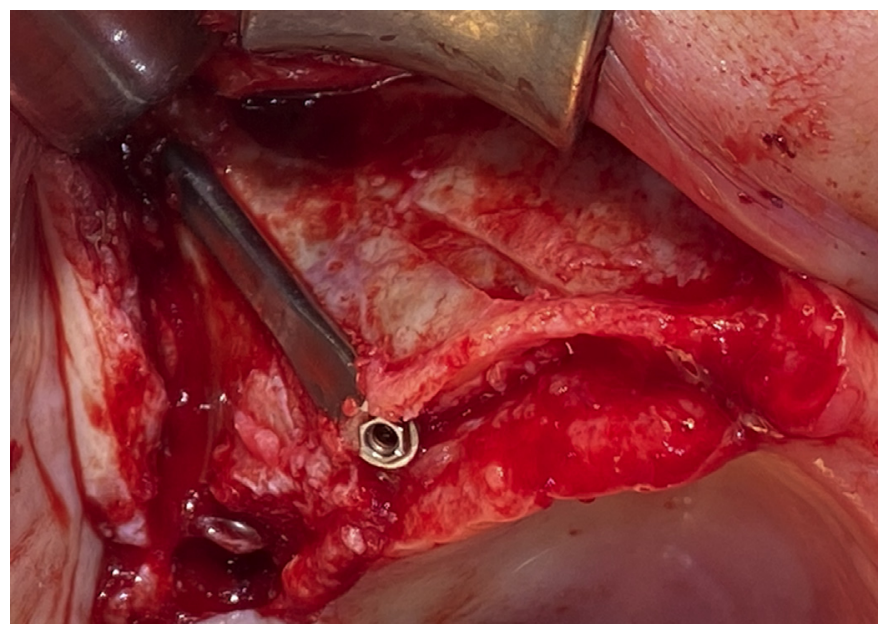

Fig. 81 Case 3: the conservative ZAGA channel-type osteotomy together with an adapted to the anatomy implant design (Straumann ZAGA Flat) prevent eventual complications such as oralantral fistula or soft tissue dehiscence.

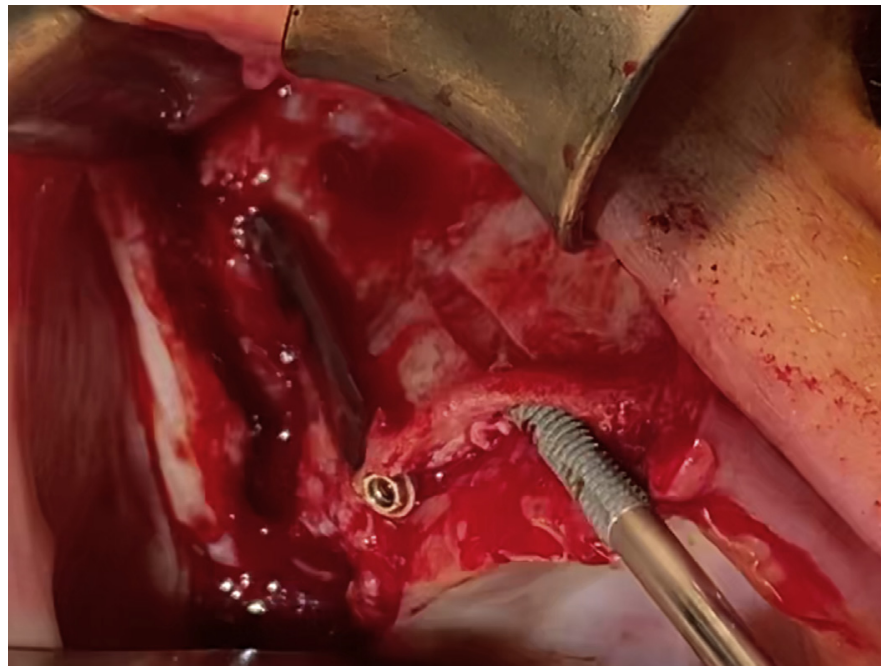

Fig. 83 Case 3: a circular implant section design (Straumann ZAGA Round) is facing a ZAGA tunnel-type osteotomy.

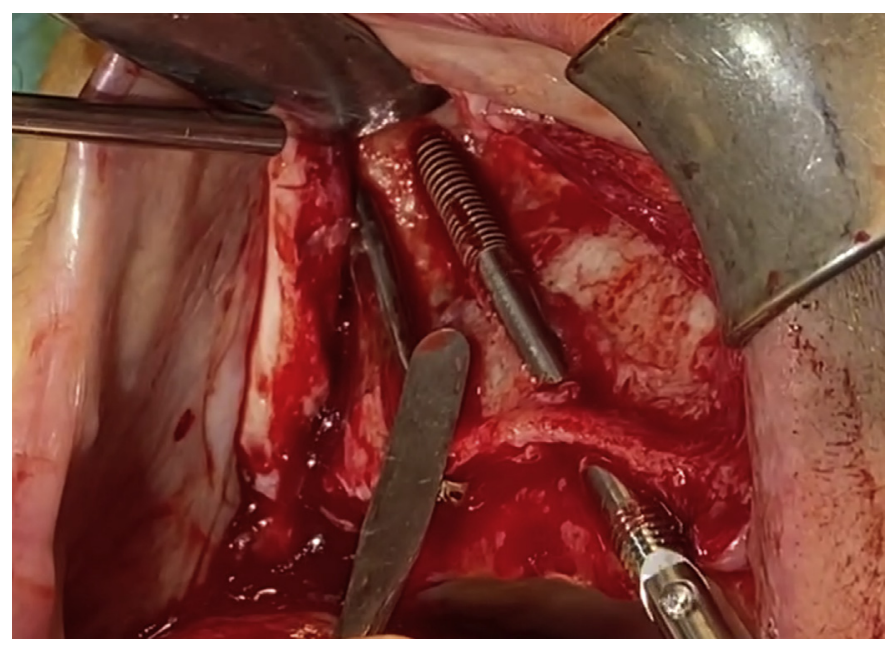

Fig. 85 Case 3: maxillary bone is so thin that an instrument is needed to avoid its complete fracture during insertion of the implant (Straumann ZAGA Round). 


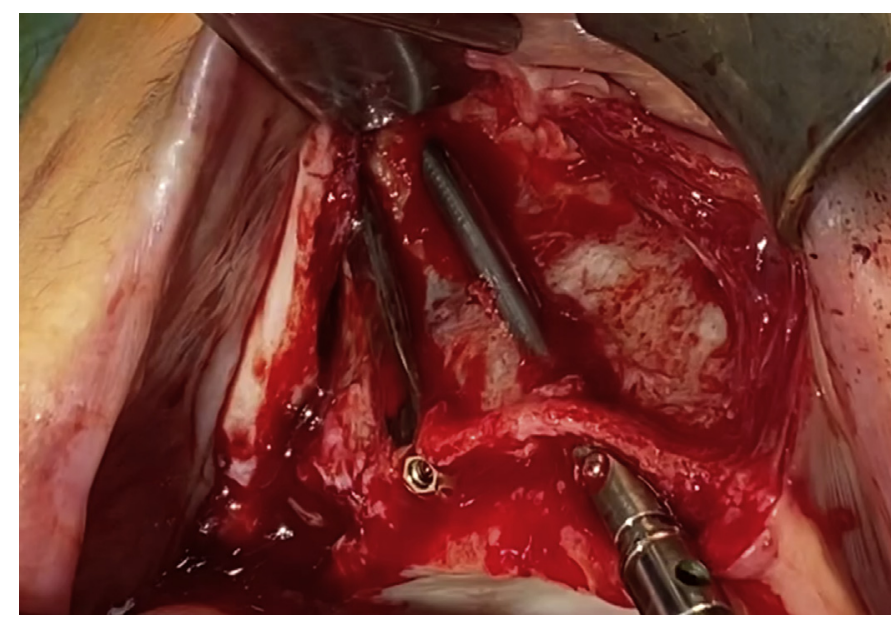

Fig. 86 Case 3: the Straumann ZAGA Round implant has been inserted. Implant mount is not interfering with hard bone usually found at the nasal spine level.

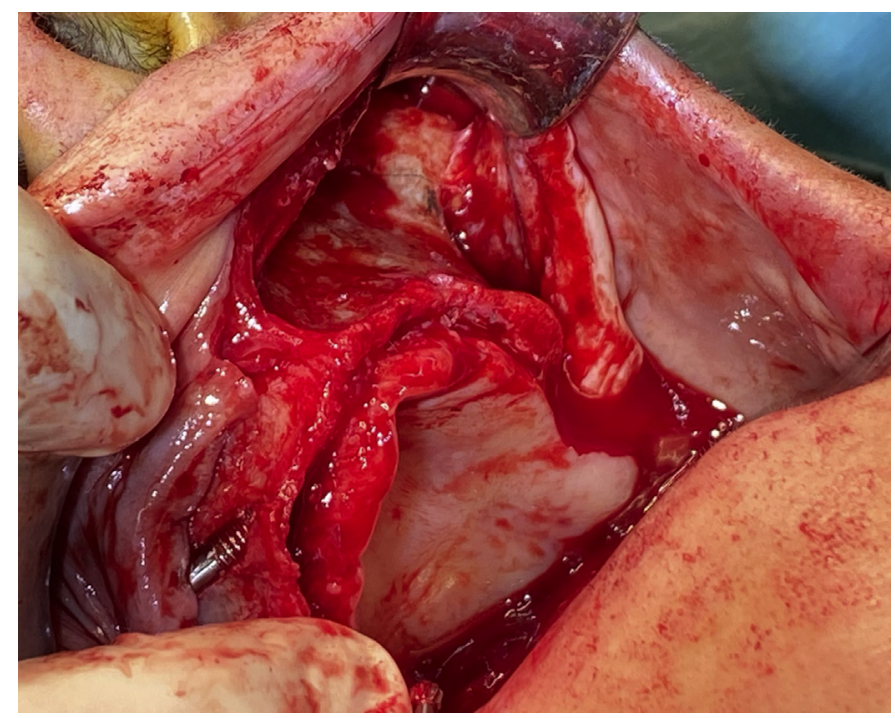

Fig. 88 Case 3: for complete control, the retractor must be in place with a double function: to protect skin from drill injury and drilling direction indicator.

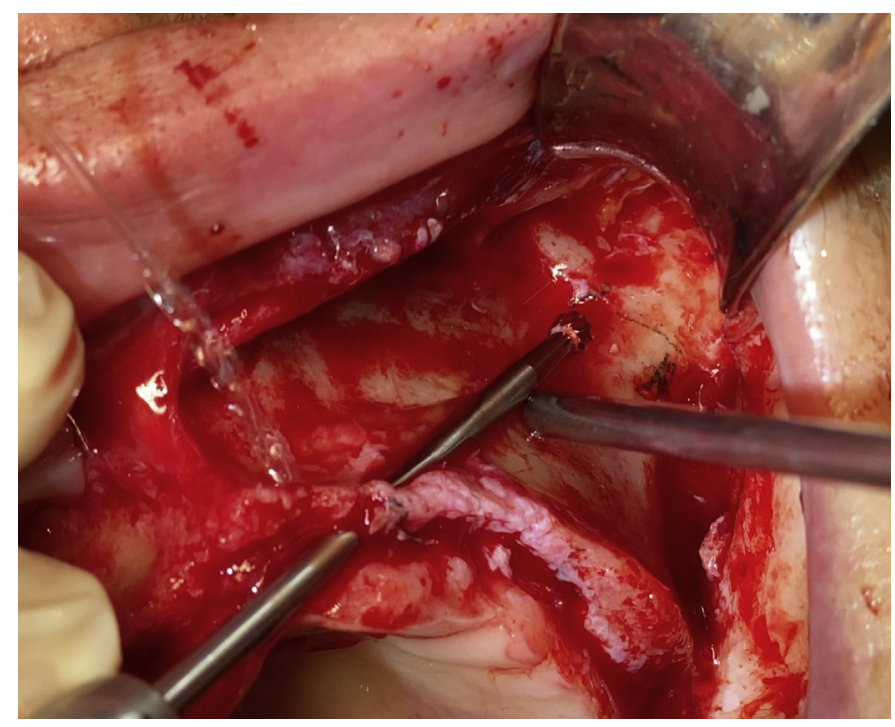

Fig. 90 Case 3: round bur direction is progressively changed into a more horizontal angulation.

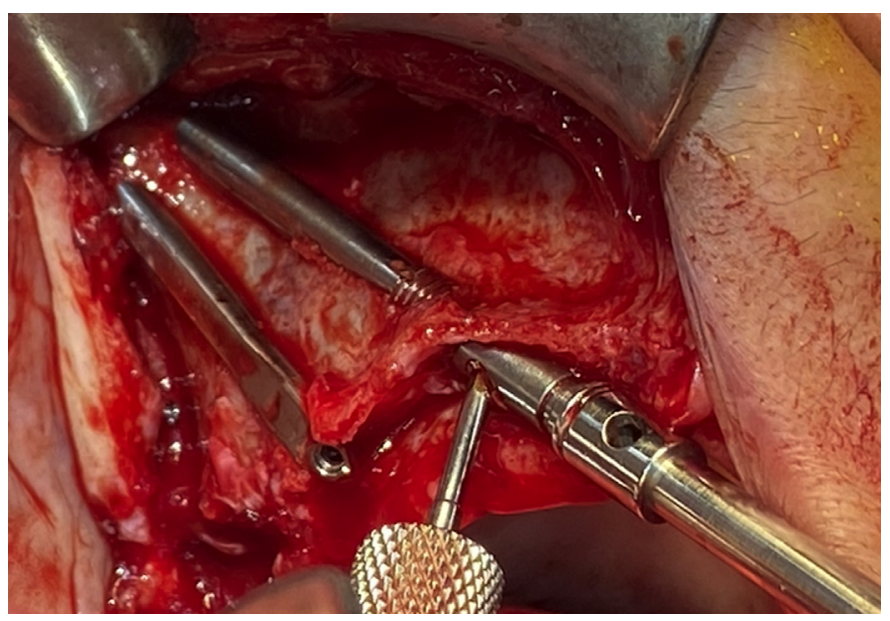

Fig. 87 Case 3: the screwdriver has been inserted on the screw fixing the mount to the implant. Screwdriver direction is indicating the prosthetic screw emergence.

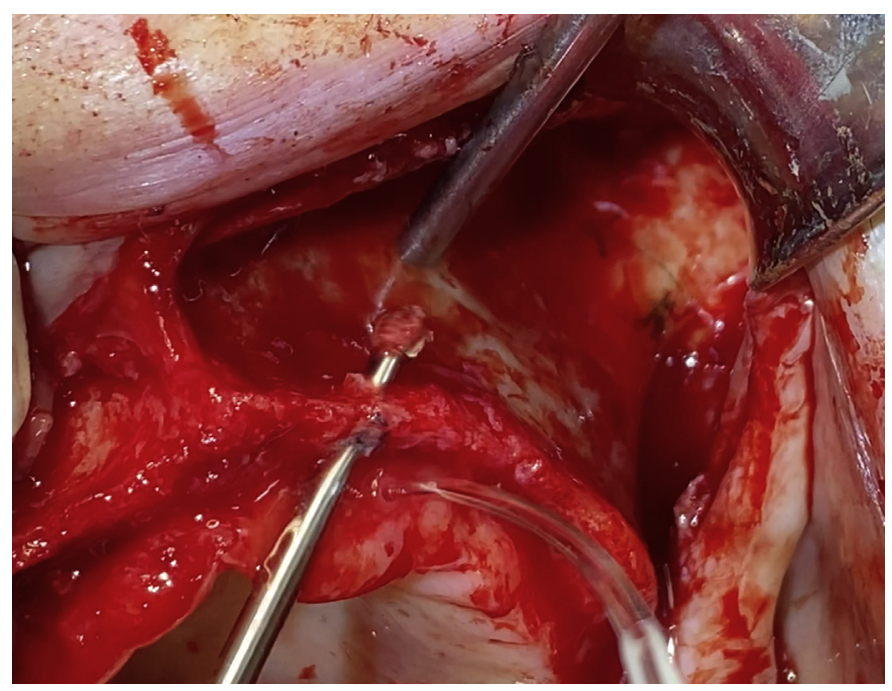

Fig. 89 Case 3: round bur is used here as the first drill. Drilling direction is facially oriented to prevent nasal or sinus perforation.

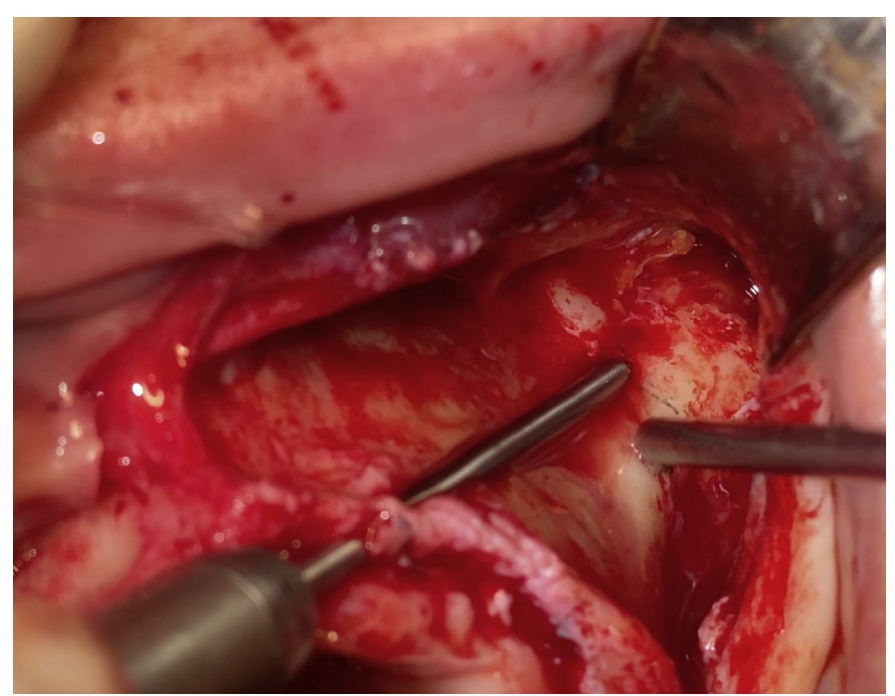

Fig. 91 Case 3: round bur has initiated the antrostomy. 


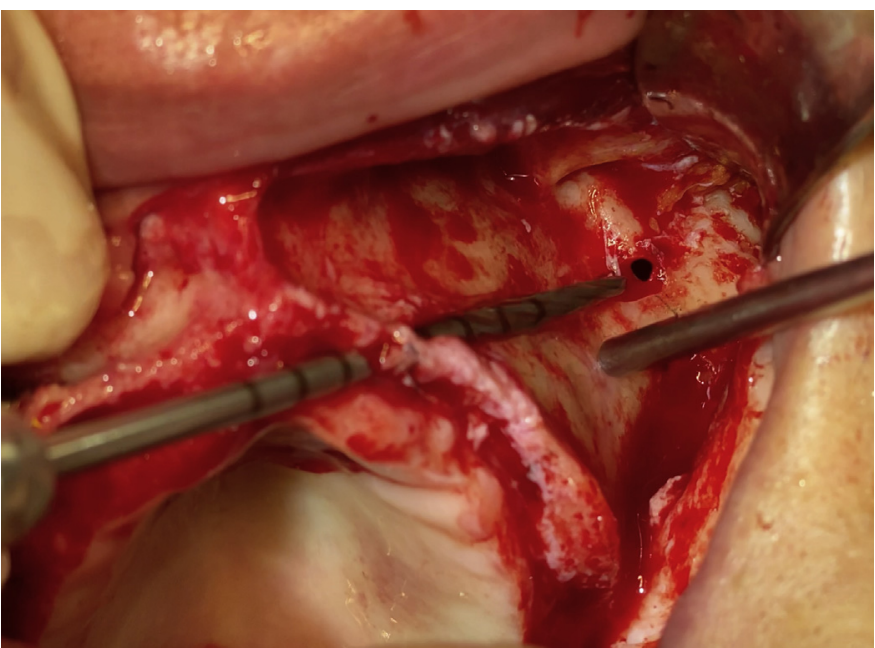

Fig. 92 Case 3: a zygo-bur (Versah.com) was used to achieve a deeper and uniform osteotomy in both alveolar and wall maxillary bone.

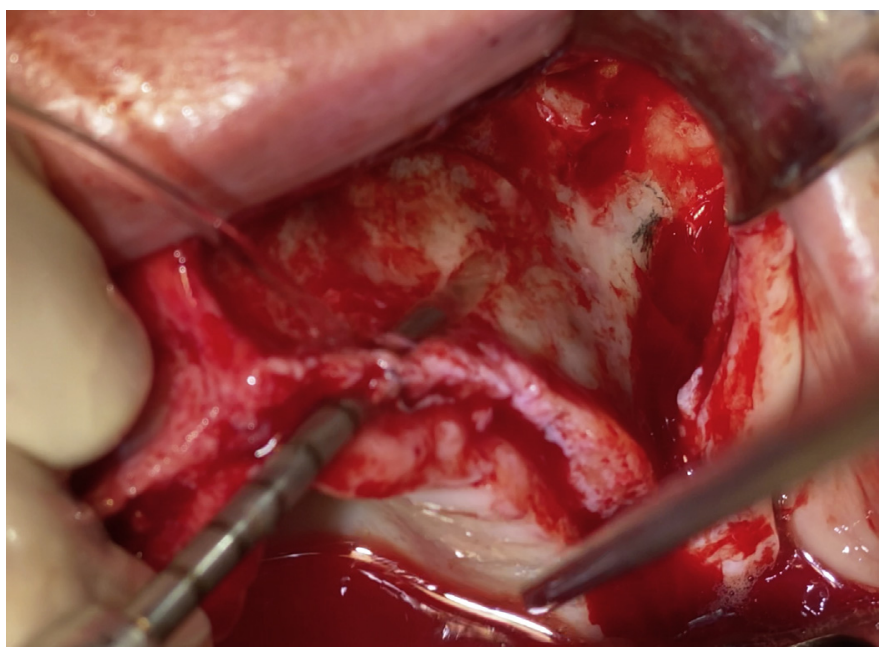

Fig. 94 Case 3: a long Versah zygo-drill was used for a complete smoothing of the initial osteotomy so that the final twist drill slides for and back while precisely maintaining the correct direction.

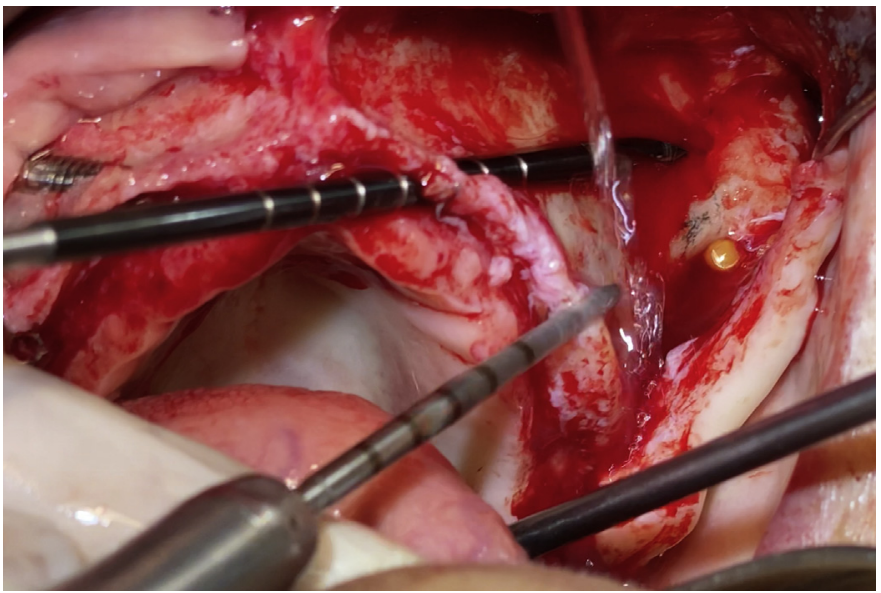

Fig. 96 Case 3: a short zygo-bur (versah.com) is used in a lateral cutting function to start the ZAGA channel-type osteotomy of the posterior left implant.

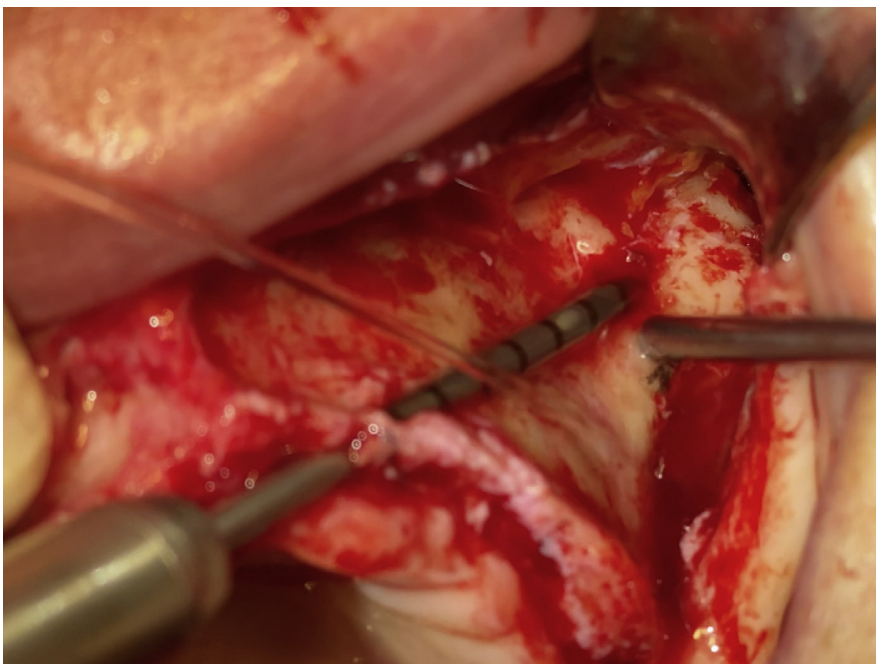

Fig. 93 Case 3: the bur (Versah.com) is used for lateral tunnel and channel carving until the total implant diameter can be hosted or the sinus lining is transparent through the osteotomy. If the latter occurs, the achieved depth should be considered optimal for the present situation.

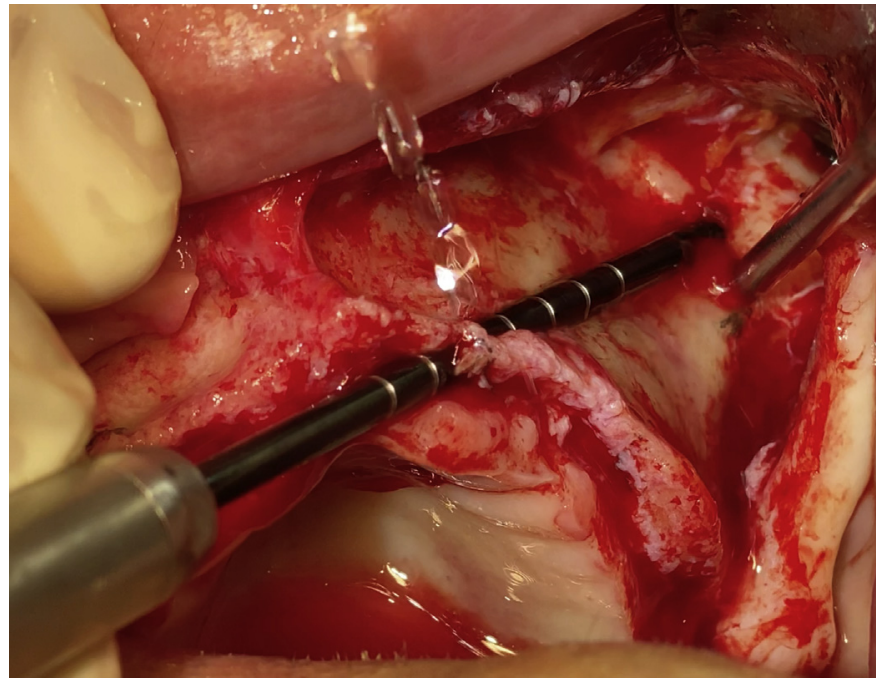

Fig. 95 Case 3: a 2.8-mm diameter twist drill was used to complete the anterior left osteotomy. The height of the alveolar process was not enough for an ideal tunnel-type osteotomy. Most of the implant circumference, however, will be bony embraced.

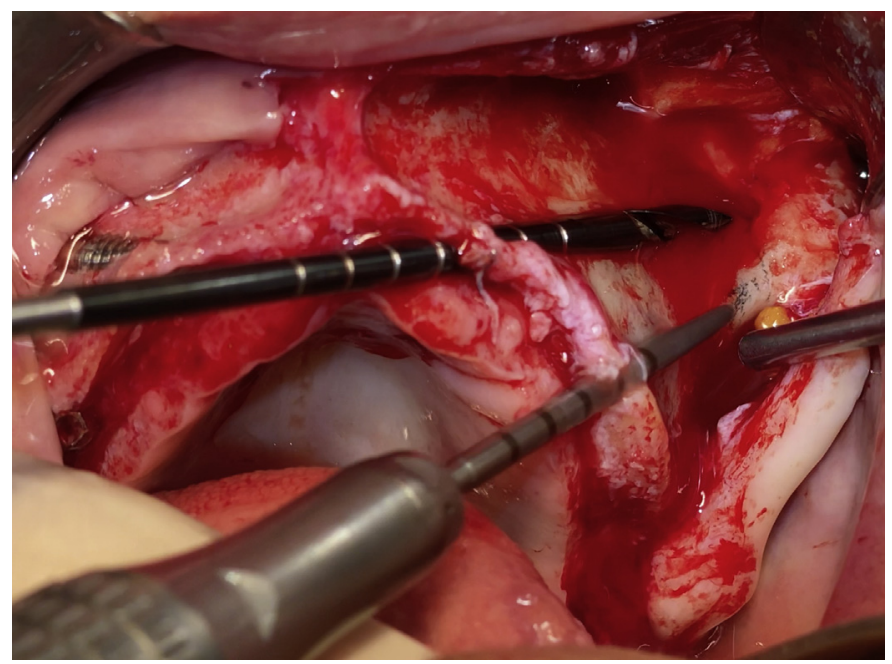

Fig. 97 Case 3: the antrostomy zone mark is guiding the direction of the drill while carving an uniform channel able to guide the next drill. 


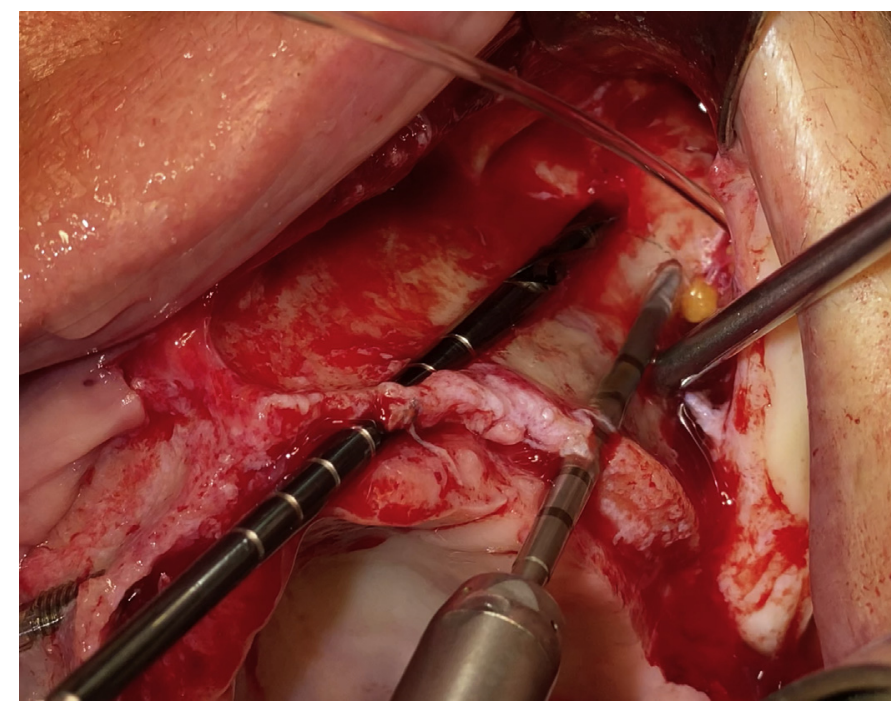

Fig. 98 Case 3: being used in a clockwise direction Versah zygobur cutting tip may start or even perform the antrostomy. Case 3: after creating an uniform channel with the desired depth, the antrostomy has been initiated using a Versah zygo-bur.

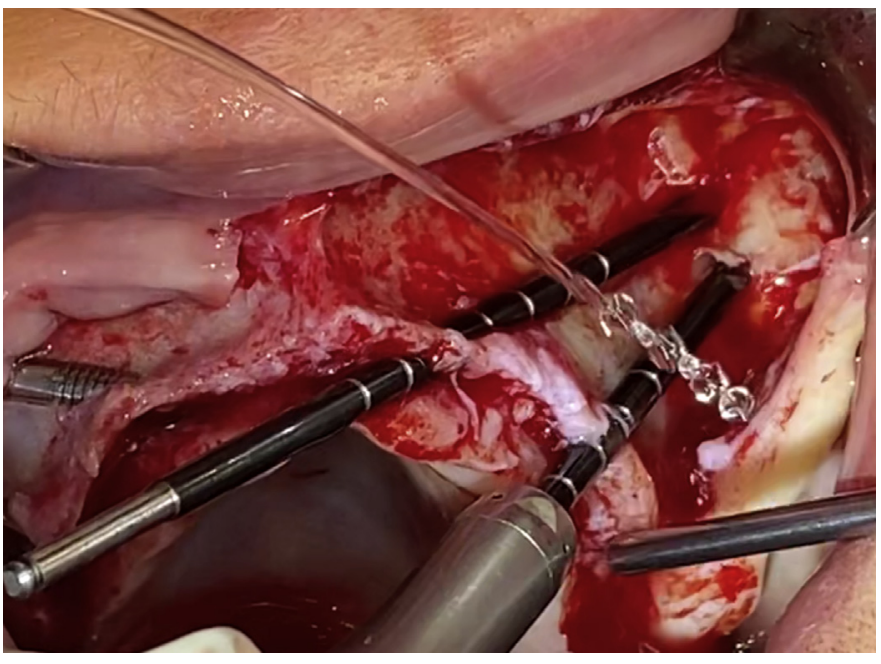

Fig. 100 Case 3: due to the poor zygomatic bone quality and quantity a $2.8-\mathrm{mm}$ diameter twist drill was considered to substitute for the usual $2.9-\mathrm{mm}$ diameter.

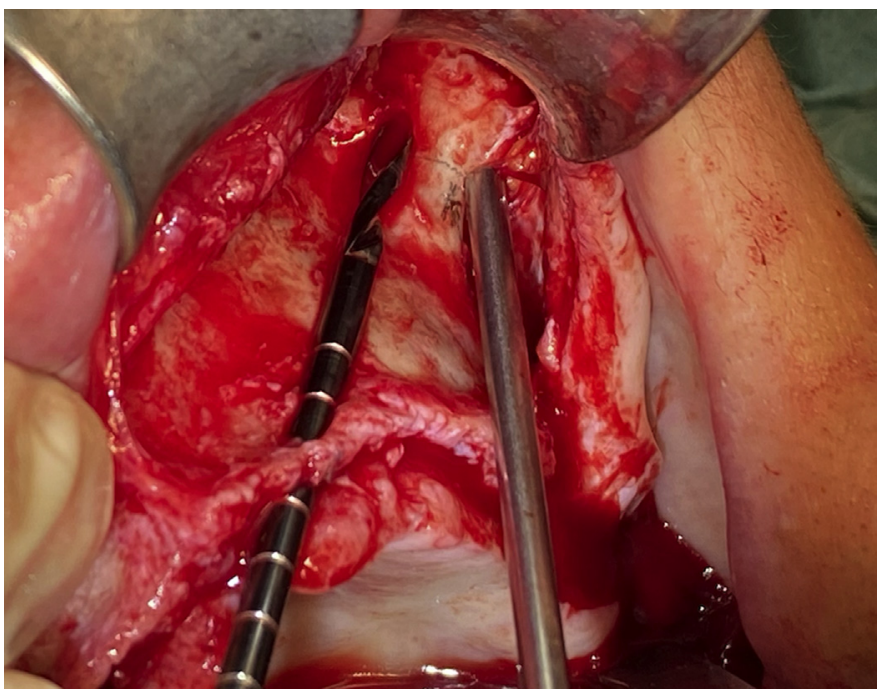

Fig. 102 Case 3: posterior osteotomy length is being measured.

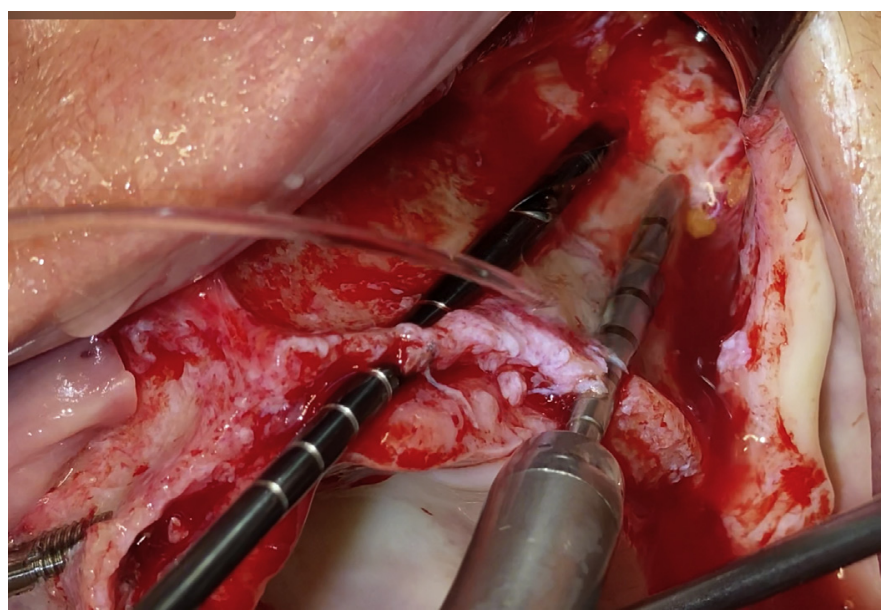

Fig. 99 Case 3: a wider diameter of Versah zygo-drill is used to enlarge the osteotomy.

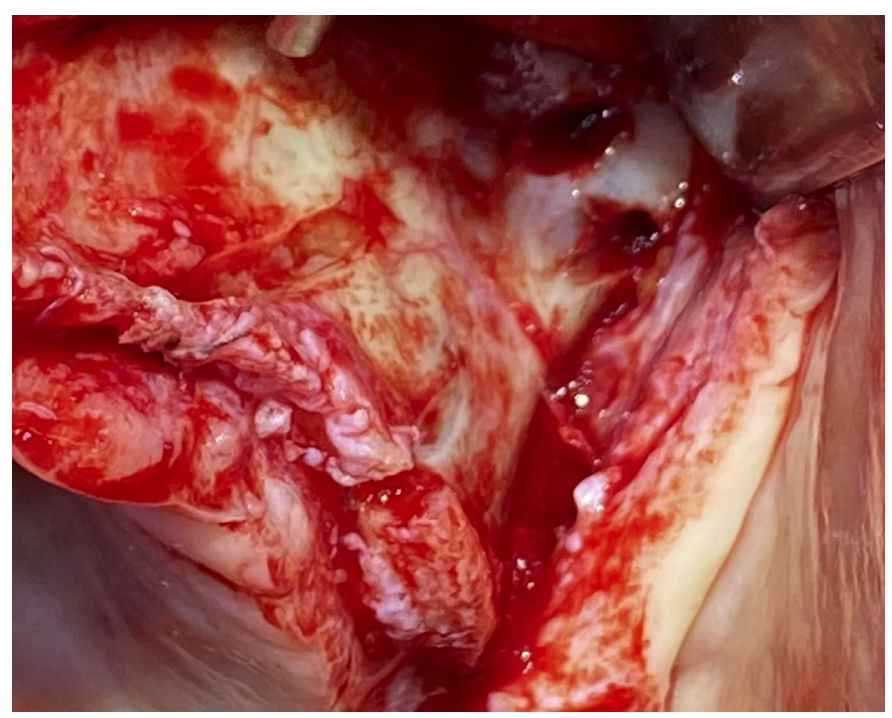

Fig. 101 Case 3: transparency of the sinus membrane is observed on both osteotomies. The ZAGA minimally invasive osteotomy concept does not need for window or slot aggressive osteotomies.

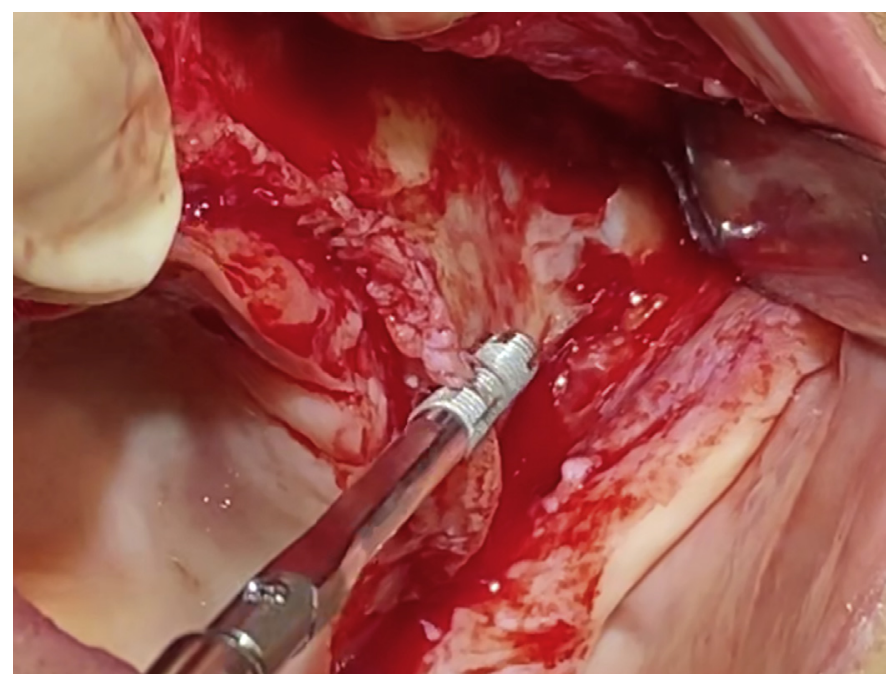

Fig. 103 Case 3: although there is no roof the implant (Straumann ZAGA Flat) is being inserted as in the case of a tunnel osteotomy. The implant self-tapping characteristic will help for an intimate BIC at the alveolar level. 


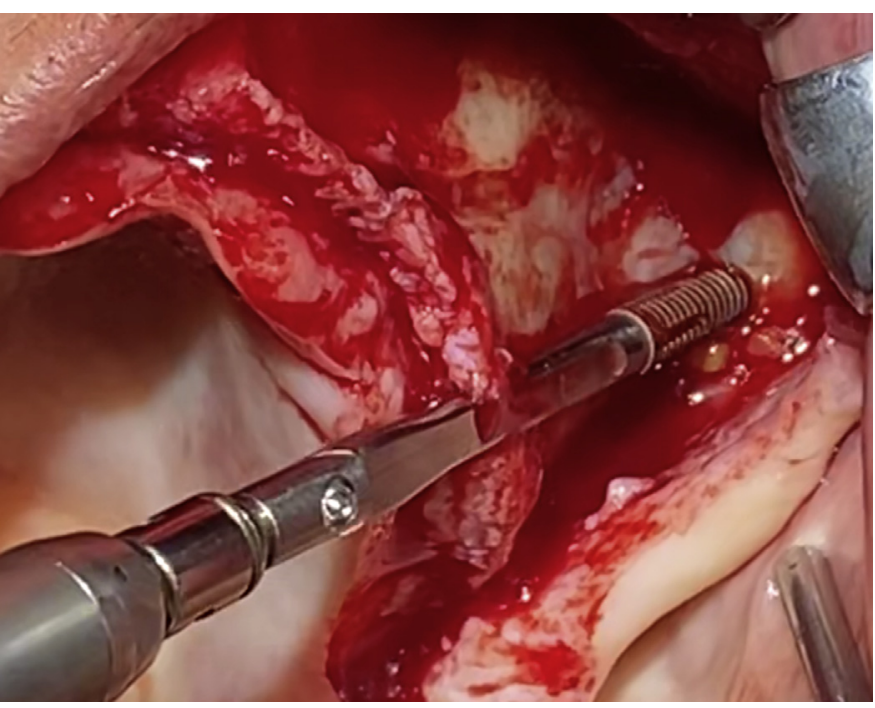

Fig. 104 Case 3: following a precise slightly underprepared osteotomy, the implant (Straumann ZAGA Flat) self-tapping feature makes possible an enhanced BIC not only at the zygomatic anchoring zone but also at the maxillary wall and at the ZICZ.

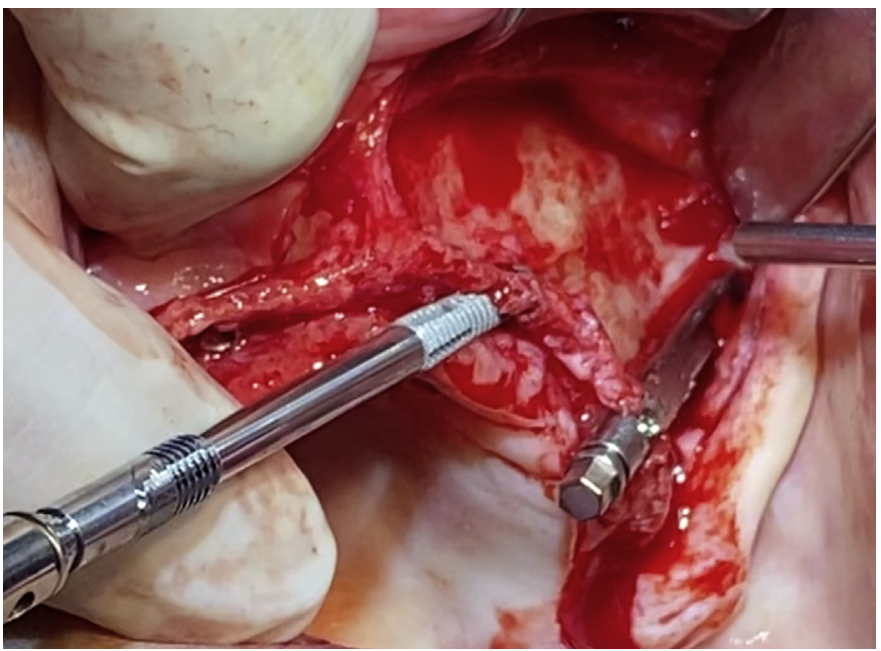

Fig. 106 Case 3: the implant (Straumann ZAGA Round) is facing the tunnel-type osteotomy.

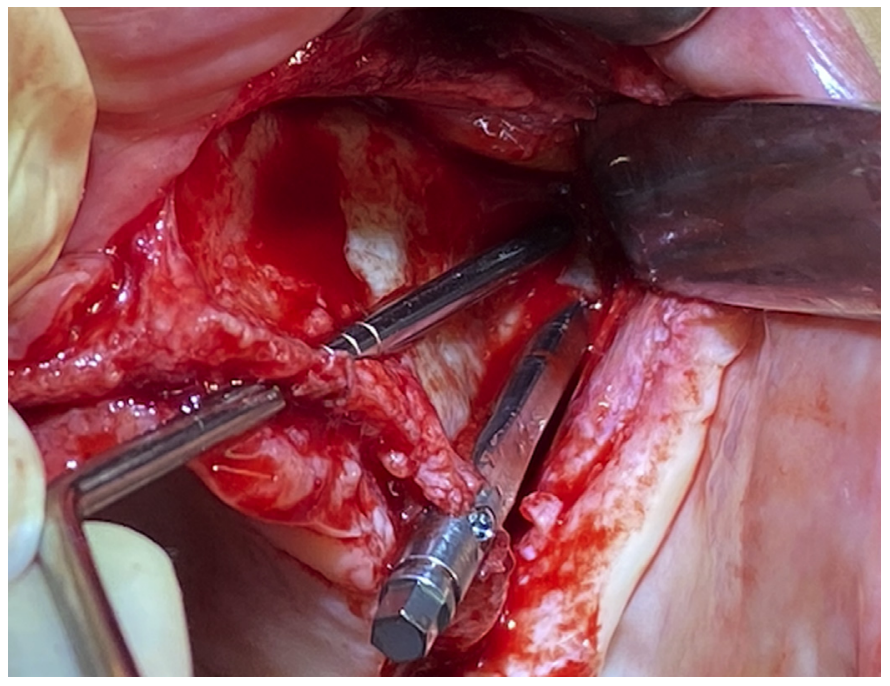

Fig. 105 Case 3: ZAGA Flat posterior left implant has been screwed. It is time for anterior osteotomy length measurement.

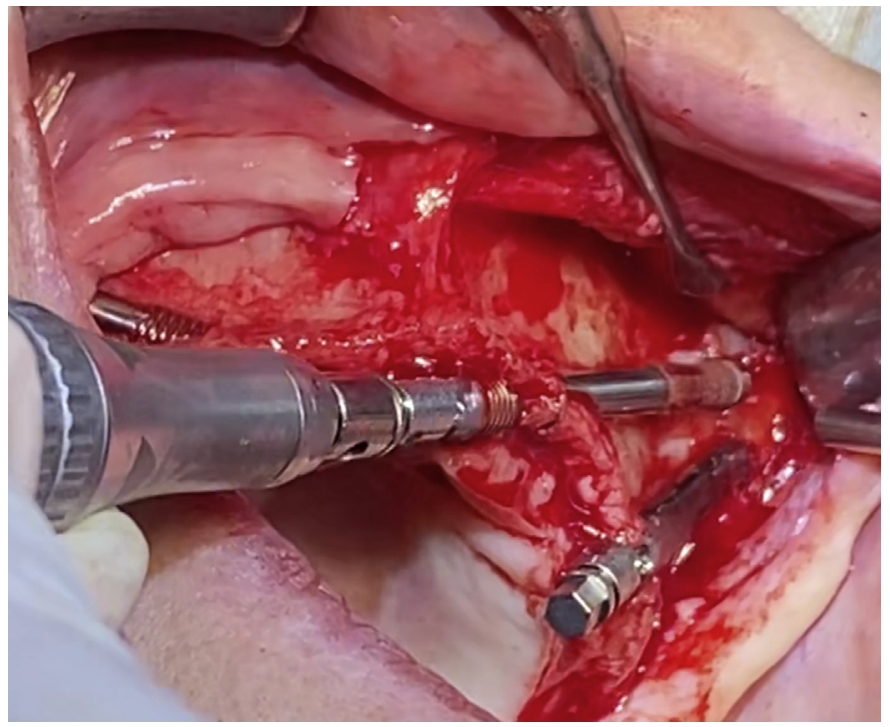

Fig. 107 Case 3: ZAGA Round implant apical flutes are selftapping the zygomatic anchoring zone. Neck flutes are self-tapping the alveolar tunnel osteotomy.

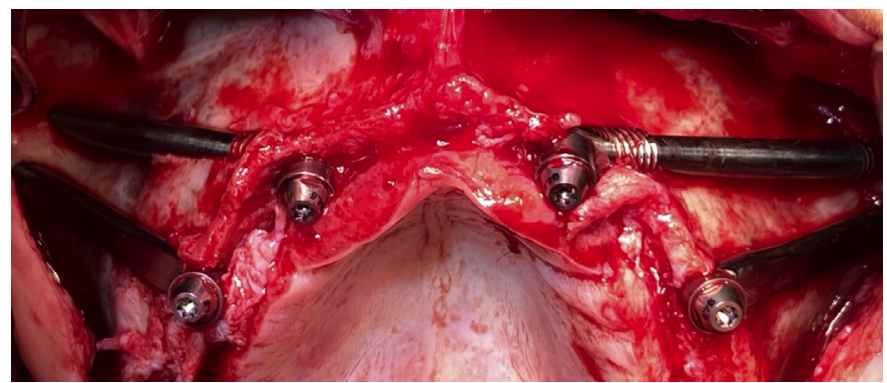

Fig. 108 Case 3: implants in place. Straumann zygomatic abutments have been screwed using $35 \mathrm{~N} \cdot \mathrm{cm}$. 


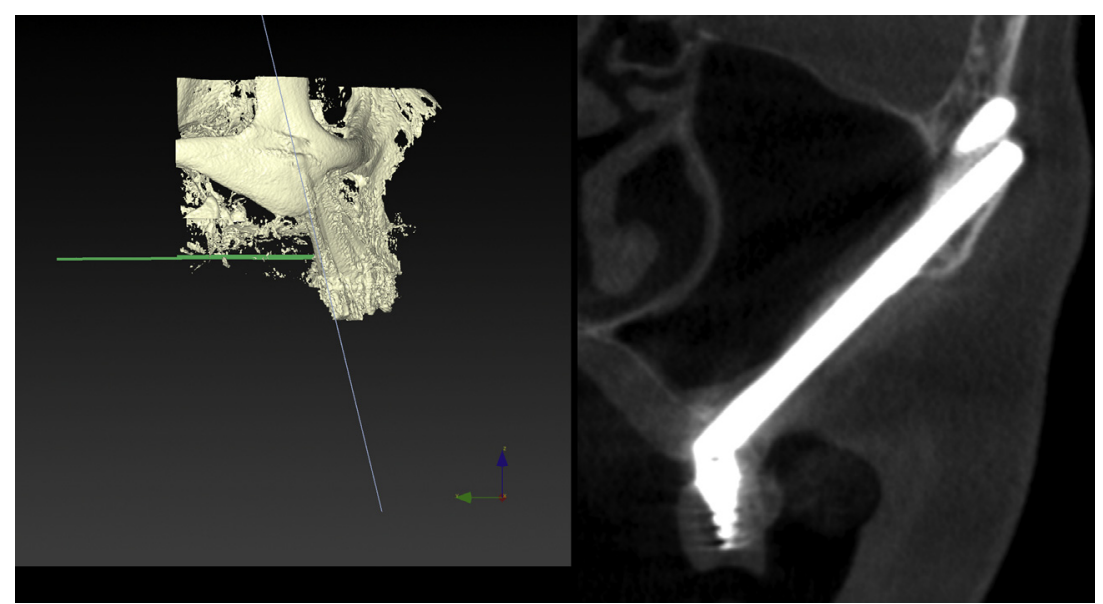

Fig. 109 Case 3: postoperative CBCT cut of the posterior right implant; compare with Fig. 58.

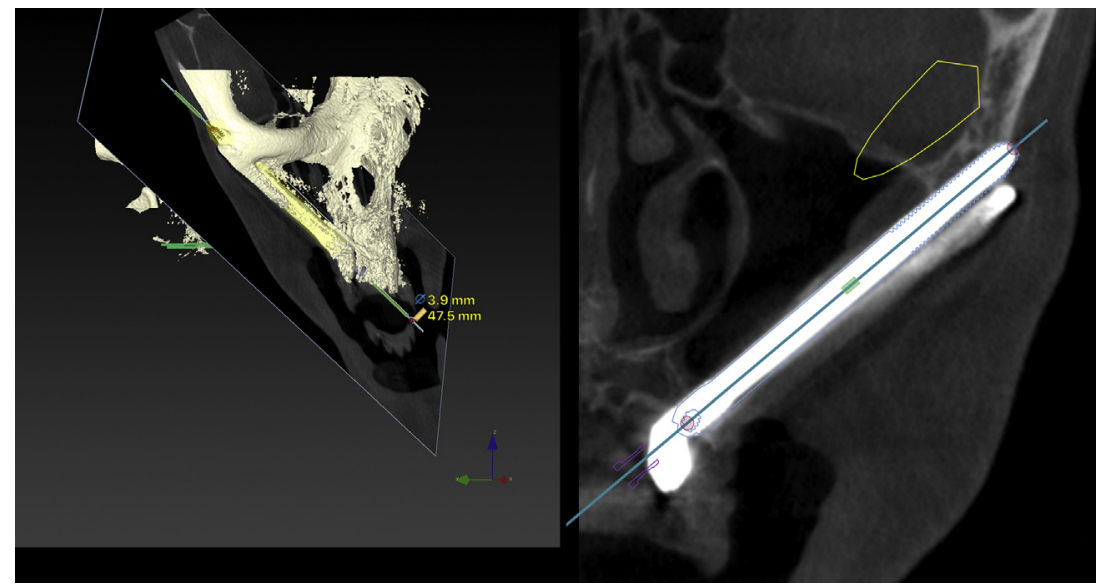

Fig. 110 Case 3: postoperative CBCT cut of the anterior right implant; compare with Fig. 59.

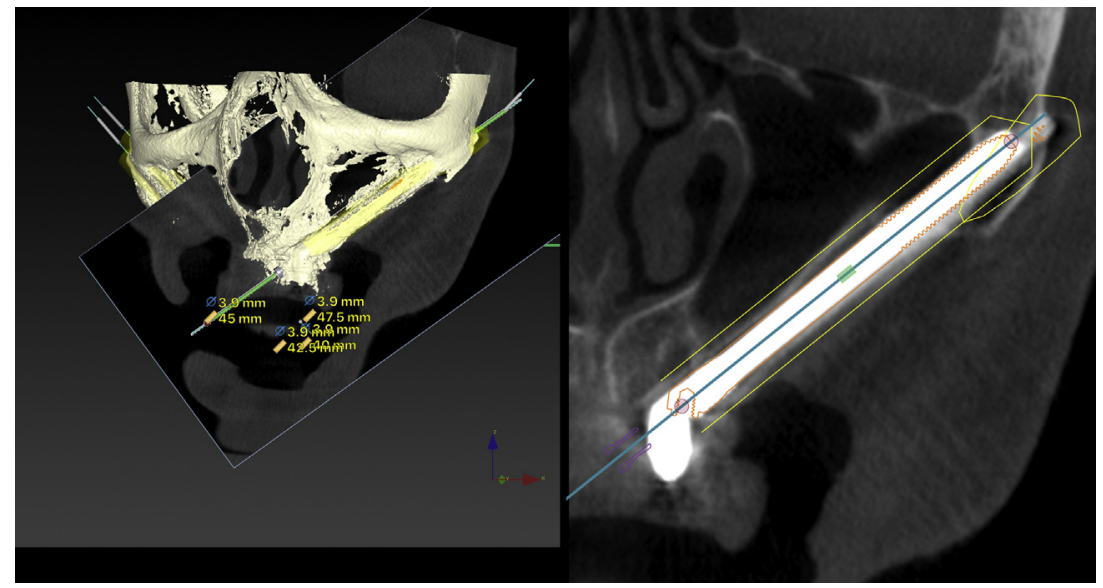

Fig. 111 Case 3: postoperative CBCT cut of the anterior left implant; compare with Fig. 60. 


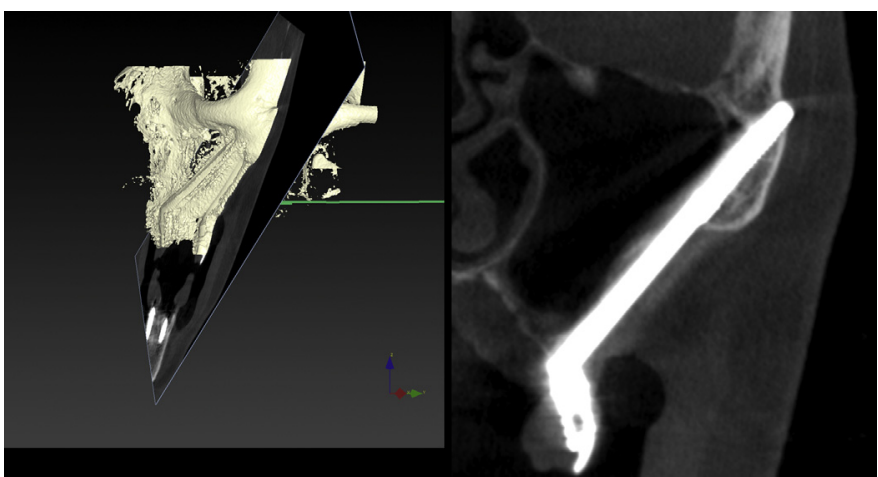

Fig. 112 Case 3: postperative CBCT cut of the posterior left implant; compare with Fig. 61.

micromovements, quality of soft tissue attachment, type of oral hygiene maintenance, history of periodontitis, parafunctional habits, smoking, and so forth. In other words, if substantial circumferential alveolar bony support at the $\mathrm{ZI}$ platform may be maintained, this should be the first option.

\section{The alveolar and/or maxillary wall channel osteotomy for a flat section implant design}

The clinical scenario of the severely atrophic maxilla is represented by a thin $(\leq 2-\mathrm{mm})$ bone separating the maxillary sinus from the overlying soft tissue. An eventual implant entry scarcely achieves BIC, and the maintenance of weak soft tissue

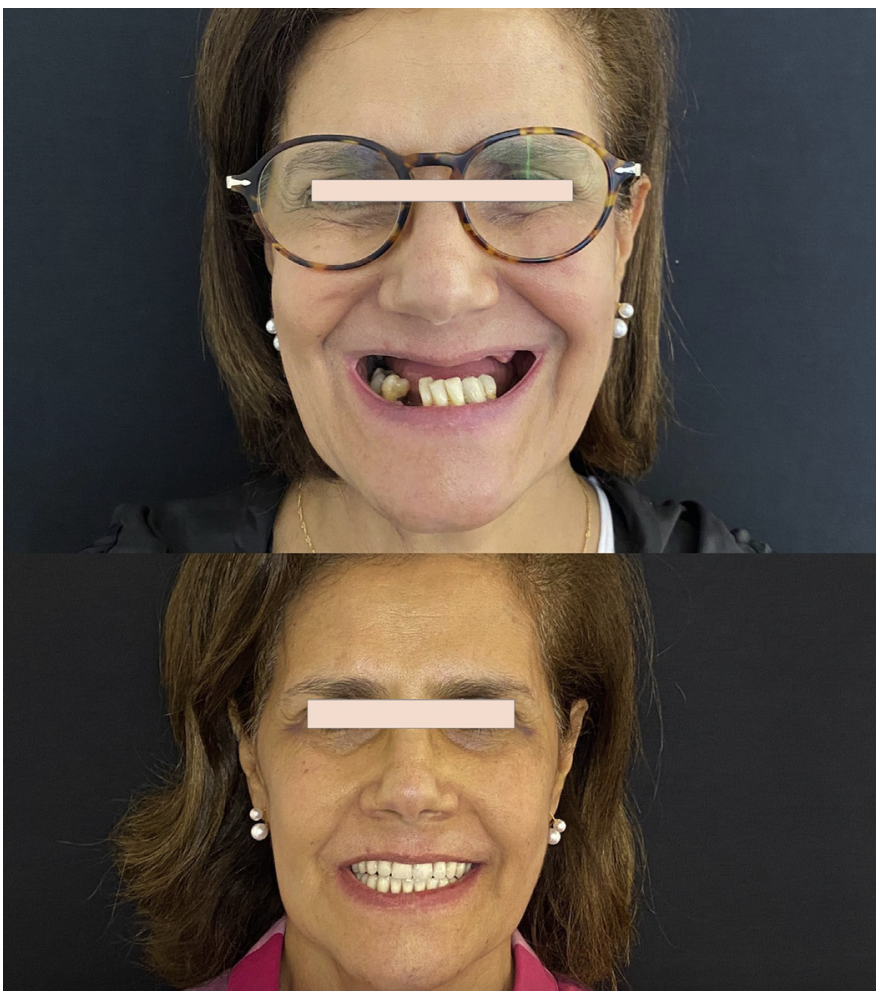

Fig. 113 Case 3: patient status after prosthesis placement. Prosthetic rehabilitation executed by Drs Alexandra Marquez and Marina Praetere, Lisbon, Portugal. sealing becomes critical. Under these conditions, osseointegration of the $\mathrm{Zl}$ at its neck level is difficult to achieve and to maintain. Risk for late sinus-oral communication then is increased.

Instead of perforating through an inadequate crestal or palatal bone, the ZAGA concept promotes displacing the alveolar antrostomy toward the buccal plate or vestibule as far as possible from the zygomatic implant critical zone (ZICZ). The main goals for this variation are to avoid sinus late complications by maintaining sinus membrane integrity at the alveolar level; the secondary goal is to prevent soft tissue dehiscence, over the body of the implant along the remaining alveolar bone and maxillary wall. A channel-type space capable of accommodating most of the circumference of the implant helps prevent soft tissue dehiscence by positioning the body of the implant appropriately within the soft tissue tolerance.

This type of osteotomy, not capable of providing a complete covering of the implant midbody and neck, is defined as a channel osteotomy. It is a groove made on the coronal-alveolar bone and sometimes also in the lateral maxillary wall and zygomatic buttress. Like a waterway or channel, it has a floor and lateral walls with more or less height, but no roof. When a channel osteotomy is performed, bone is not covering the buccal implant midpart or its neck.

A bony canal having a circumferential arc section ideally is sealed by an implant showing a circumferential arc section too. Accordingly, a recommendation is made to use a flat implant section design that fits into a channel minimizing its buccal impact against soft tissue.

The final idea of the channel type of osteotomy (see Figs. 21-55 and 56-113) is to sink the implant neck as much as possible on the alveolar and lateral wall maxillary bone and still not perforate the sinus membrane. The rationale for this ZAGA concept recommendation is based on the fact that in patients with minimal bone thickness around the implant's entry point, under function and in time, bone may resorb. As a consequence, sinus sealing is achieved just by a weak hemidesmosome junction. That fragile antrum sealing may be jeopardized in many circumstances-inadequate hygiene, use of water jet oral hygiene devices against the gingival junction, inappropriate or aggressive use of periodontal probe, and so forth. It has been speculated that the lack of bony support would end up in transversal mobility of the long coronal part of the $\mathrm{Zl}$ facilitating oral-sinus communication. On the contrary, placing the Zl platform partially or outside of the sinus maintaining lateral maxillary bony support/contact would allow for better prosthetic positioning together with a more conservative approach regarding sinus integrity preservation.

In such an extremely resorbed anatomy model, a strong recommendation is made to, if possible, position the antrostomy at least $15 \mathrm{~mm}$ above the coronal area of the platform (Figs. 57-61 and Figs. 109-112). It also is recommended to preserve the sinus membrane integrity and as much bone thickness as possible at the ZICZ crest level. To prevent soft tissue stretching when placing the implant laterally to the maxillary wall, it is recommended to groove buccal bone to house the implant body with as much medial submergence as possible into bone crest in such a way that it does not protrude against periosteum, jeopardizing soft tissue vascularity. The depth limit for the canal formation is dictated by maintenance of the sinus membrane integrity at this level. 


\section{Clinics care points}

- The first step is to balance the 2 main late risks of the treatment-oral-antral communication and soft tissue dehiscence-and decide the type of osteotomy.

- Typical zygomatic surgery is not an emergency surgery: wait for complete soft tissue healing previously to the surgery.

- Use a palatal incision to move keratinized soft tissue buccally to the implant head.

- Consider using a machined surface for the implant neck and body. If dehiscence occurs, it is easy to maintain.

- It is mandatory to preserve the sinus membrane integrity and as much bone thickness as possible at the ZICZ crest level.

\section{Acknowledgments}

The authors would like to acknowledge the remarkable work in the planning and prosthetic rehabilitation of patients reported in Figs. $1-20$ and Figs. $21-113$ by Drs Peter and Madalina Simon, from the Stuttgart ZAGA Center, and in by Drs Alexandra Marquez and Marina Praetere, from the Lisbon MD Center
Straumann AG generously donated most of the implants shown on the pictures, while Versah.com kindly donated the Zygo-Osseocondensation drills; however described ZAGA protocols properties belong to the authors, and Straumann and Versah by no means interfered with this publication concepts.

\section{Disclosure}

C. Aparicio is the founder of the ZAGA Centers Network. He is an inventor of the patent describing the ZAGA implant zygomatic portfolio. W.D. Polido is a frequent speaker for the Straumann Group and Geistlich companies.

\section{References}

1. Aparicio C, editor. Zygomatic implants: the anatomy-guided approach. 1st edition. United Kingdom: Quintessence Pub Co; 2012.

2. Al-Nawas B, Wegener J, Bender C, et al. Critical soft tissue parameters of the zygomatic implant. J Clinperiodontol 2004;31(7): 497-500.

3. Becktor JP, Isaksson S, Abrahamsson P, et al. Evaluation of 31 zygomatic implants and 74 regular dental implants used in 16 patients for prosthetic reconstruction of the atrophic maxilla with cross-arch fixed bridges. Clin Implant Dent Relat Res 2005;7: 159-65. 